$\operatorname{Argonne} \underset{\bigotimes}{\bigotimes}$

ANL/EVS/AGEM/TR-09-04

\title{
October 2008 Monitoring Results for Barnes, Kansas
}

\author{
Environmental Science Division
}


About Argonne National Laboratory

Argonne is a U.S. Department of Energy laboratory managed by UChicago Argonne, LLC under contract DE-AC02-06CH11357. The Laboratory's main facility is outside Chicago, at 9700 South Cass Avenue, Argonne, Illinois 60439. For information about Argonne and its pioneering science and technology programs, see www.anl.gov.

\section{Availability of This Report}

This report is available, at no cost, at http://www.osti.gov/bridge. It is also available on paper to the U.S. Department of Energy and its contractors, for a processing fee, from:

U.S. Department of Energy

Office of Scientific and Technical Information

P.O. Box 62

Oak Ridge, TN 37831-0062

phone (865) 576-8401

fax (865) 576-5728

reports@adonis.osti.gov

\section{Disclaimer}

This report was prepared as an account of work sponsored by an agency of the United States Government. Reference herein to any specific commercial product, process, or service by trade name, trademark, manufacturer, or otherwise, does not necessarily constitute or imply its endorsement, recommendation, or favoring by the United States Government or any agency thereof. The views and opinions of document authors expressed herein do not necessarily state or reflect those of the United States Government or any agency thereof, Argonne National Laboratory, or UChicago Argonne, LLC. 


\section{October 2008 Monitoring Results for Barnes, Kansas}

by

Applied Geosciences and Environmental Management Section

Environmental Science Division, Argonne National Laboratory

February 2009

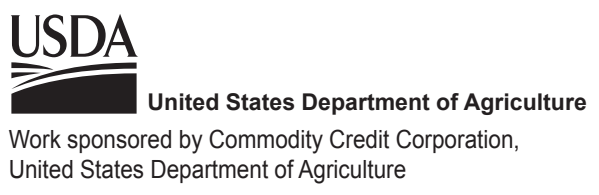




\section{Contents}

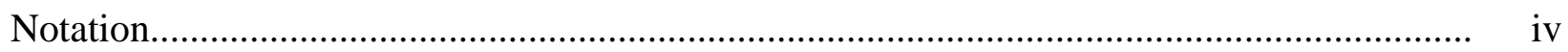

1 Introduction and Background ……………….............................................................. 1-1

2 Sampling and Analysis Activities............................................................................ 2-1

2.1 Measurement of Groundwater Levels.................................................................... 2-1

2.2 Monitoring Well Sampling and Analyses............................................................ 2-2

2.3 Handling and Disposal of Investigation-Derived Waste ......................................... 2- 2-4

2.4 Quality Control for Sample Collection, Handling, and Analysis .............................. 2-4

3 Results and Discussion ............................................................................................ 3-1

3.1 Groundwater Level Data............................................................................... 3-1

3.2 Analytical Results for Volatile Organic Compounds in Groundwater Samples and Lateral Distribution of the Contaminants............................................................... 3-2

3.3 Vertical Distribution of Carbon Tetrachloride and Evaluation of Data to Distinguish Aquifer Zones by Depth ............................................................................ 3-4

4 Conclusions and Ongoing Tasks........................................................................................ 4 4-1

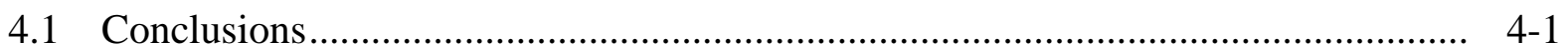

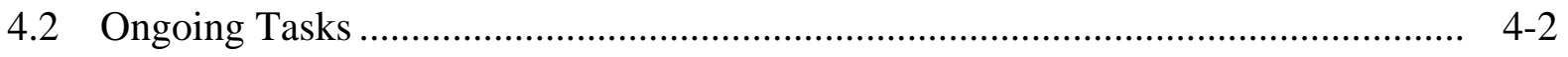

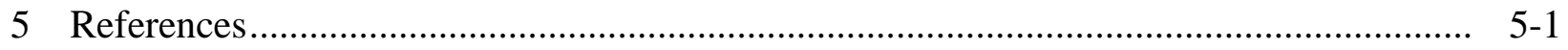

Appendix A: Sampling Activities and Field Measurements at Barnes in October 2008 ....... A-1

Appendix B: Analytical Results for Wastewater Samples from Pace Analytical Services, Inc ......................................................................................... B-1

Appendix C: Results from the AGEM Laboratory for Dual Analyses of Samples Collected at Barnes in October 2008 and for Quality Control Samples ............................. C-1

Appendix D: Sample Documentation from TestAmerica Laboratories, Inc. ……………...... D-1

Appendix E: Results of City of Barnes Sampling of Public Wells in 1986-2008 ……......... E-1

\section{Figures}

1.1 Groundwater sampling locations at Barnes in October 2008 ....................................... 1-2

2.1 Wells at Barnes equipped with data loggers for water level monitoring, prior to the November 2008 download event.

2.2 Wells at Barnes equipped with data loggers for future water level monitoring, after the Novemer 2008 installation of five new recorders and the removal of others. 


\section{Figures (cont.)}

3.1 Potentiometric surface maps depicting the groundwater flow direction at Barnes in December 2007 and May 2008, before pumping of the public water supply wells and during pumping

3.2 Hydrographs summarizing monthly results of long-term water level monitoring in wells at Barnes, January to November 2008.

3.3 Analytical results for carbon tetrachloride in groundwater samples collected at Barnes in November 2007 to October 2008

3.4 Analytical results for chloroform in groundwater samples collected at Barnes in November 2007 to October 2008.

3.5 Interpreted carbon tetrachloride plume in October 2008 in wells screened in the intermediate aquifer zone.

3.6 Interpreted carbon tetrachloride plume in October 2008 in wells screened in the deep aquifer zone

\section{Tables}

3.1 Hand-measured water levels at Barnes, March-November 2008

3.2 Analytical results from the AGEM Laboratory for volatile organic compounds in groundwater samples collected at Barnes, July 2006 to October 2008 ....

A.1 Sequence of sampling activities at Barnes in October 2008.

A.2 Field measurements for groundwater samples collected at Barnes, July 2006 to October 2008.

C.1 Analytical results for samples and replicates collected at Barnes in October 2008 and for quality control samples 


\section{Notation}

AGEM Applied Geosciences and Environmental Management

AMSL above mean sea level

BGL below ground level

${ }^{\circ} \mathrm{C} \quad$ degree(s) Celsius

CCC Commodity Credit Corporation

COC chain of custody

DO dissolved oxygen

EPA U.S. Environmental Protection Agency

$\mathrm{ft} \quad$ foot (feet)

gal gallon(s)

$\mathrm{hr} \quad$ hour(s)

KDHE Kansas Department of Health and Environment

L liter(s)

$\mu \mathrm{g} / \mathrm{L} \quad$ microgram(s) per liter

$\mu \mathrm{S} / \mathrm{cm} \quad$ microsiemen(s) per centimeter

$\mathrm{mg} / \mathrm{L} \quad$ milligram(s) per liter

min minute

$\mathrm{mV} \quad$ millivolt(s)

ORP oxidation-reduction potential

PWS public water supply

RBSL risk-based screening level

TOC top of casing

USDA U.S. Department of Agriculture

VOC volatile organic compound 


\section{October 2008 Monitoring Results for Barnes, Kansas}

\section{Introduction and Background}

The Commodity Credit Corporation of the U.S. Department of Agriculture (CCC/USDA) operated a grain storage facility at Barnes, Kansas, during most of the interval 1949-1974. Carbon tetrachloride contamination was initially detected in 1986 in the town's public water supply wells. In 2006-2007, the CCC/USDA conducted a comprehensive targeted investigation at and near its former property in Barnes to characterize this contamination. Those results were reported previously (Argonne 2008a).

In November 2007, the CCC/USDA began quarterly groundwater monitoring at Barnes. The monitoring is being conducted on behalf of the CCC/USDA by Argonne National Laboratory, in accord with the recommendations made in the report for the 2006-2007 targeted investigation (Argonne 2008a). The objective is to monitor the carbon tetrachloride contamination identified in the groundwater at Barnes. The sampling is presently conducted in a network of 28 individual monitoring wells (at 19 distinct locations), 2 public water supply wells, and 1 private well (Figure 1.1).

The results of the 2006-2007 targeted investigation and the subsequent monitoring events in November 2007 (Argonne 2008b), March 2008 (Argonne 2008c), and July 2008 (Argonne 2008d) demonstrated the presence of carbon tetrachloride contamination in groundwater at levels exceeding the Kansas Department of Health and Environment (KDHE) Tier 2 risk-based screening level (RBSL) of $5.0 \mu \mathrm{g} / \mathrm{L}$ for this compound. The contaminant plume appears to extend from the former CCC/USDA property northwestward, toward the Barnes public water supply wells. Information obtained during the 2006-2007 investigations indicates that at least one other potential source might have contributed to the groundwater contaminant plume (Argonne 2008a). The former agriculture building owned by the local school district, located immediately east of well PWS3, is also a potential source of the contamination.

This current report presents the results of the fourth monitoring event, conducted in October 2008. During this fourth monitoring event, low-flow sampling methods were used to purge and sample all wells. This was the third event at Barnes during which low-flow sampling methods were used. 


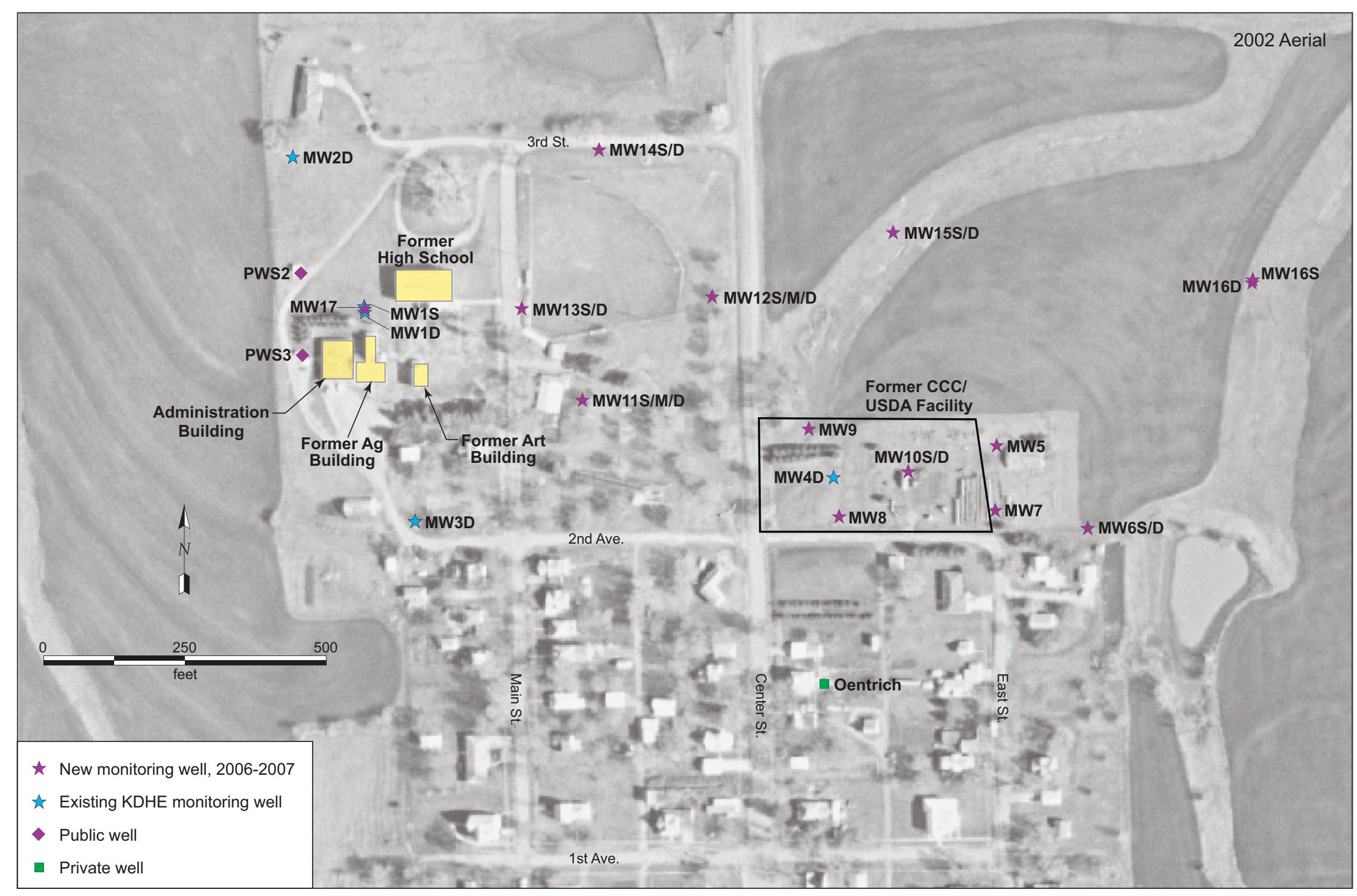

FIGURE 1.1 Groundwater sampling locations at Barnes in October 2008. Source of photograph: NAPP (2002). 


\section{Sampling and Analysis Activities}

\subsection{Measurement of Groundwater Levels}

The groundwater sampling event at Barnes on October 22-24, 2008, involved 28 monitoring wells (MW1S, MW1D, MW2D, MW3D, MW4D, MW5, MW6S, MW6D, MW7, MW8, MW9, MW10S, MW10D, MW11S, MW11M, MW11D, MW12S, MW12M, MW12D, MW13S, MW13D, MW14S, MW14D, MW15S, MW15D, MW16S, MW16D, MW17). All of the well locations are shown in Figure 1.1. A chronological summary of the field activities is in Appendix A, Table A.1.

Before implementation of the low-flow sampling described in Section 2.2, a hand-held water level indicator was used to measure the depth to groundwater and the total depth of each well, to within $0.01 \mathrm{ft}$, from the top of the well casing. Monitoring wells MW1S and MW12S were measured but were found to be dry and consequently could not be sampled. Two public water supply wells (PWS2 and PWS3) and one private well (Oentrich) were sampled but could not be measured because of well construction issues. The hand-measured water level data are presented in Section 3.1 and discussed further in Section 3.3.

In addition to the manual water level measurements, data recorders have been gathering long-term data on the groundwater elevation and gradient at monitoring wells MW1D, MW2D, MW3D, MW4D, MW7, MW9, MW14D, MW15D, and MW16D and at the Oentrich private well (Figure 2.1). With the exception of the Oentrich well, automated monitoring of these wells continued during the current review period, and the recorders were downloaded on November 19, 2008. (Automated monitoring of the Oentrich well was discontinued on May 9, 2008, because of changes in the well surface completion implemented by the property owner, which prevent the installation of a recorder.) The data loggers record water levels continuously at 60-min intervals.

During the download on November 19, 2008, problems were discovered that had caused the following data recorders to stop working:

- The recorder in well MW3D was found to be damaged, probably because of water infiltrating into the well vault. This was the fourth water level recorder that had failed at the MW3D location, because of water damage, since 
automated water level monitoring was initiated in July 2006. The recorder was removed and will be replaced as soon as a replacement is delivered from the manufacturer. The data stored in the recorder could not be retrieved.

- The recorder in well MW7 failed to collect data after being reprogrammed in July 2008. After battery replacement on November 19, 2008, the recorder appeared to be working normally and was reinstalled.

- The recorder in well MW1D failed because of irreparable electrical damage; no data could be recovered from the unit. The recorder was removed and will be replaced as soon as possible.

Additional water level recorders have been ordered by Argonne, and the damaged units at wells MW1D and MW3D will be replaced as soon as possible.

Water level recorders were installed in five wells - MW10S, MW11M, MW12M, MW13S, and MW17 (Figure 2.2) — at the time of the November download event. These locations were prioritized for automated monitoring to investigate the potential hydraulic influences on groundwater flow and contaminant migration in the intermediate aquifer zone (as discussed in Section 3.3), in which the highest concentrations of carbon tetrachloride in groundwater have been identified. Upon completion of the November 2008 downloads and new installations, a total of 12 water level recorders are in place at the locations shown in Figure 2.2.

The automatically recorded groundwater level data are presented and discussed in Section 3.1.

\subsection{Monitoring Well Sampling and Analyses}

After measurement of water levels, low-flow groundwater sampling techniques, according to U.S. Environmental Protection Agency (EPA) guidelines (Puls and Barcelona 1996; Yeskis and Zavala 2002), were used to purge and sample the monitoring wells. The Oentrich well and the public water supply wells were sampled at their respective faucets (Table A.1 in Appendix A). The Oentrich well and public water supply well PWS2 were pumped for 5 min 
before sampling. Public water supply well PWS3 had been running for $0.5 \mathrm{~h}$, and a sample was collected without additional purging.

The low-flow sampling of monitoring wells involved the use of a bladder pump and field measurement equipment designed to determine when representative formation water was entering the well casing (Puls and Barcelona 1996; Yeskis and Zavala 2002). Stabilization of formation water in the screened area of the well was determined by measuring the static water levels and monitoring the levels of $\mathrm{pH}$, temperature, specific conductivity, oxidation-reduction potential (ORP), and dissolved oxygen (DO) during pumping.

The following procedure was followed for each well sampled:

1. A bladder pump was inserted into the well to a depth midway between the top and bottom of the screen. To minimize disturbance of the solids that are typically present at the bottom of a well, care was taken not to lower the pump to the bottom of the casing.

2. The pumping rate for the bladder pump was set to ensure that minimal drawdown occurred in each well during pumping. The rate was monitored by measuring the static water level periodically throughout pumping.

3. Polyethylene tubing was used to connect the bladder pump to an in-line flow cell. Formation parameters, including $\mathrm{pH}$, temperature, specific conductivity, ORP, and DO, were measured continuously in the in-line flow cell during pumping. Measurements were recorded every 4 min until three successive measurements for each parameter were within a range indicating that the formation water was stable. The range for formation stabilization varies for each parameter, as follows: $\mathrm{pH}$, within 0.1 ; temperature, within $3 \%$; specific conductivity, within 3\%; ORP, within $10 \mathrm{mV}$; and DO, within 10\%.

4. After stabilization of the formation water parameters occurred, the polyethylene tubing was disconnected from the in-line flow cell, and a representative groundwater sample was pumped through the tubing into laboratory-approved containers. 
5. The polyethylene tubing for each well was kept and dedicated for reuse at that well. In addition, pumping rate data were recorded for each well as a reference for subsequent sampling events.

The sequence of activities during the October 2008 well sampling event is summarized in Appendix A, Table A.1. The field measurements are in Appendix A, Table A.2.

Groundwater samples designated for analyses for volatile organic compounds (VOCs) were collected in appropriate laboratory containers, labeled, packaged, and chilled to $4^{\circ} \mathrm{C}$ by placement in ice-filled coolers. The samples were shipped via an overnight delivery service to the Applied Geosciences and Environmental Management (AGEM) Laboratory at Argonne for VOCs analyses with EPA Method 524.2 (EPA 1995). Aliquots of selected samples (chosen in the field) were also shipped to TestAmerica Laboratories, Inc., South Burlington, Vermont, for verification VOCs analyses according to EPA Contract Laboratory Program protocols.

The analytical results are presented and discussed in Section 3.2.

\subsection{Handling and Disposal of Investigation-Derived Waste}

Purge water generated as potentially contaminated investigation-derived waste was containerized on-site in 55-gal drums. The accumulated purge water was sampled and analyzed for VOCs (including ethylene dibromide) and nitrates. The analytical results (Appendix B) indicated no detectable concentrations of carbon tetrachloride, chloroform, ethylene dibromide, or nitrate. The water was discharged on-site on December 11, 2008, with the verbal approval of the KDHE.

\subsection{Quality Control for Sample Collection, Handling, and Analysis}

Quality assurance/quality control procedures followed during the October 2008 monitoring event are described in detail in the Master Work Plan (Argonne 2002). The results are summarized as follows:

- Sample collection and handling activities were monitored by the documentation of samples as they were collected and the use of chain-of- 
custody forms and custody seals to ensure sample integrity during handling and shipment.

- Samples designated for VOCs analyses were received with custody seals intact and at the appropriate preservation temperature. All samples sent to the AGEM Laboratory were analyzed within the required holding times.

- Quality control samples collected to monitor sample-handling activities (trip blanks and equipment rinsates) and method blanks analyzed with the samples to monitor analytical methodologies were all free of carbon tetrachloride and chloroform contamination.

- Groundwater samples were analyzed for VOCs at the AGEM Laboratory by the purge-and-trap method on a gas chromatograph-mass spectrometer system. Calibration checks analyzed with each sample delivery group were required to be within $\pm 20 \%$ of the standard. Surrogate standard determinations performed on samples and blanks were within the specified range of $80-120 \%$ for all samples, in either the initial analysis or a successful reanalysis.

- Results from the AGEM Laboratory for dual analyses of the groundwater samples are in Appendix C, Table C.1. The results of the dual analyses compare well, indicating consistency in the sampling and analytical methodologies. Analytical results for quality control samples are also in Appendix C, Table C.1.

- In accordance with the procedures defined in the Master Work Plan (Argonne 2002), five groundwater samples were submitted to a second laboratory (TestAmerica) for verification analysis according to the protocols of the EPA's Contract Laboratory Program. Documentation is in Appendix D. The results from the two laboratories compare well over the range of contaminant concentrations detected. Samples from monitoring wells MW3D and MW11S analyzed at AGEM Laboratory with no carbon tetrachloride detected were analyzed by TestAmerica with similar results. The low detections of carbon tetrachloride ( $<1$ to $2.1 \mu \mathrm{g} / \mathrm{L}$ ) in monitoring wells MW7 and MW17 and in 
public well PWS2 in analyses at the AGEM Laboratory were confirmed in verification analysis. 


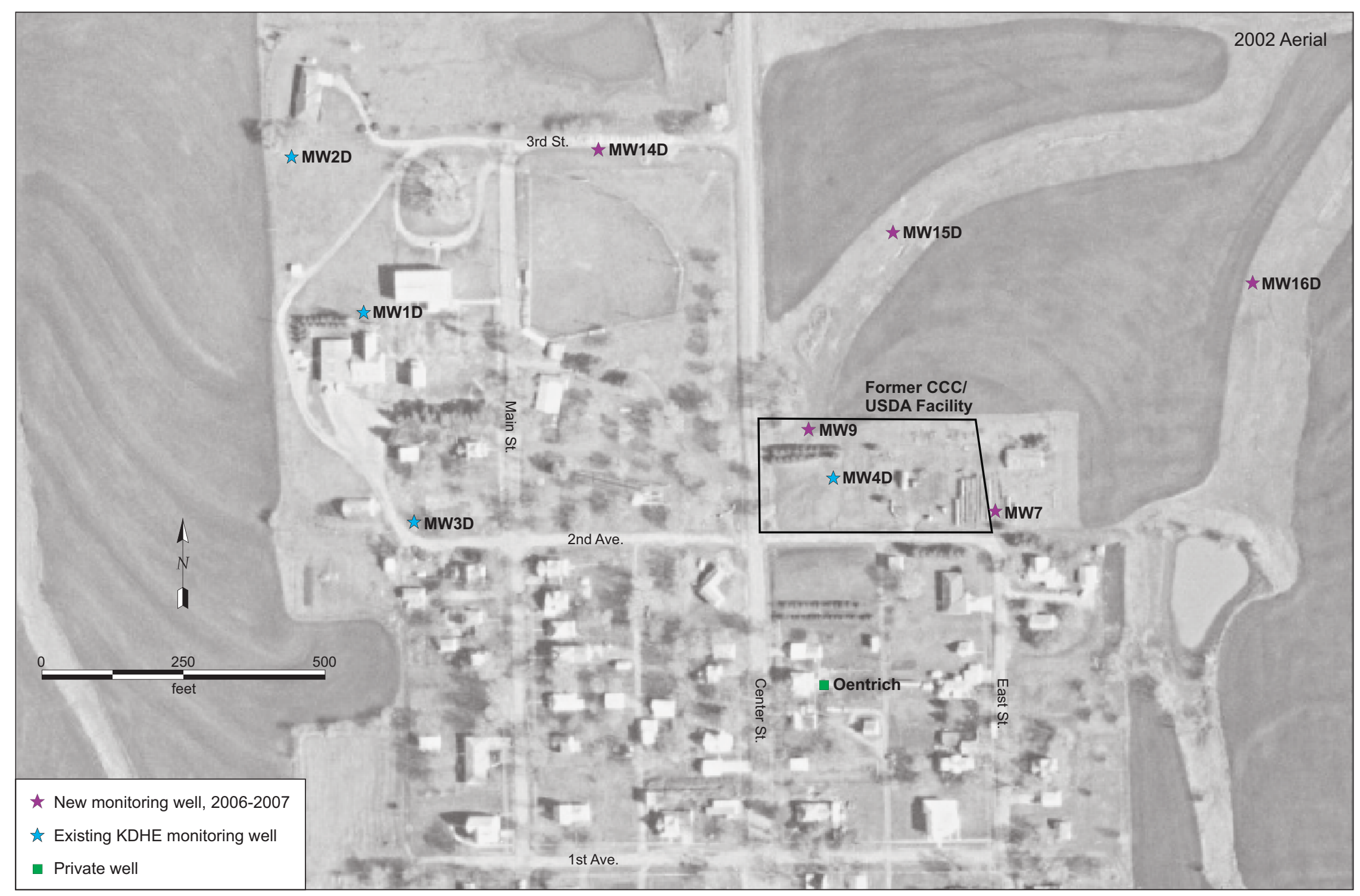

FIGURE 2.1 Wells at Barnes equipped with data loggers for water level monitoring, prior to the November 2008 download event. Source of photograph: NAPP (2002). 


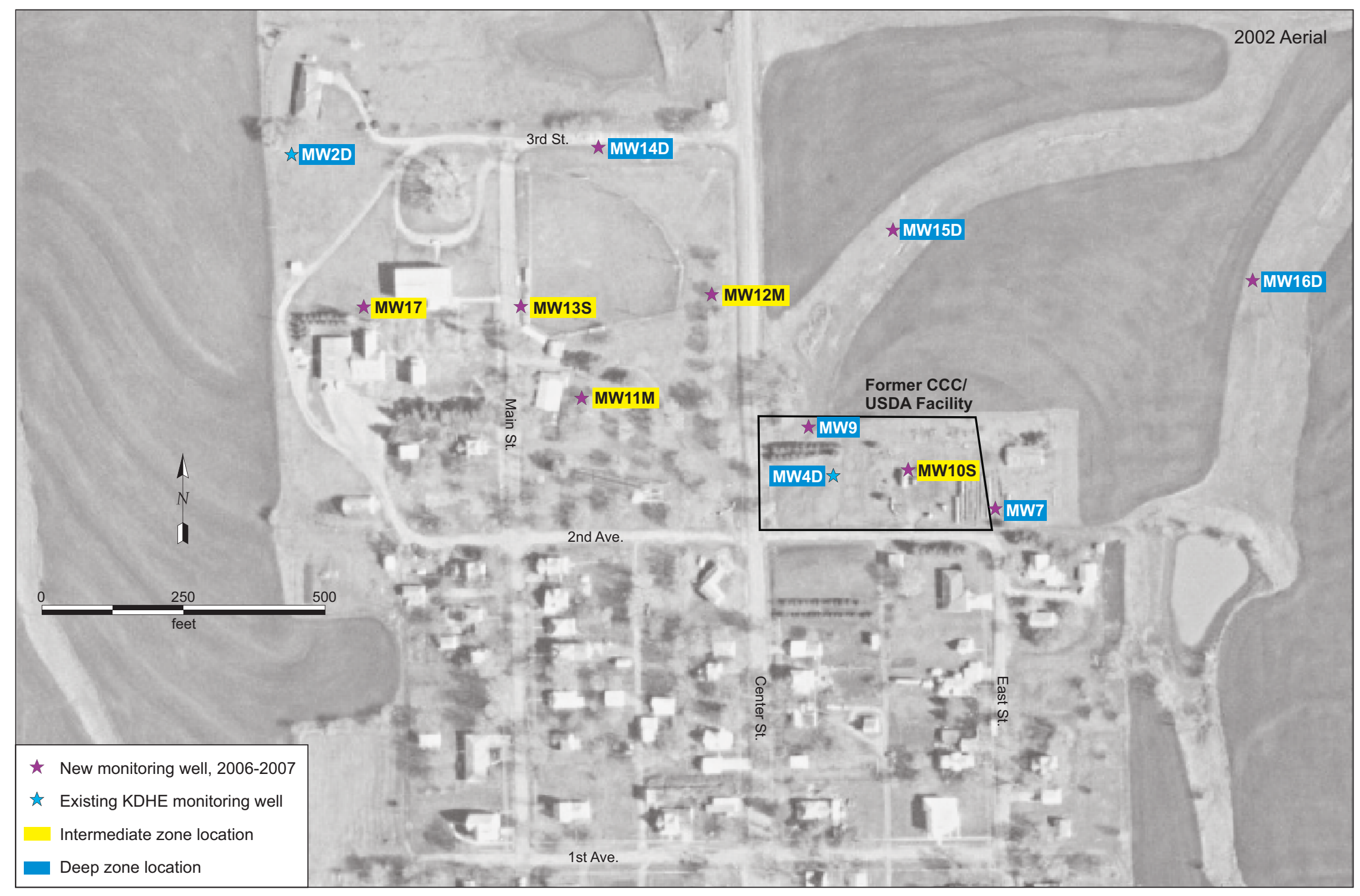

FIGURE 2.2 Wells at Barnes equipped with data loggers for future water level monitoring, after the Novemer 2008 installation of five new recorders and the removal of others. Source of photograph: NAPP (2002). 


\section{Results and Discussion}

\subsection{Groundwater Level Data}

The manual water level measurements taken during sampling on October 22-24, 2008, are in Table 3.1. These measurements are discussed in Section 3.3. Table 3.1 also includes manual water level measurements taken in March-July 2008 and November 2008.

Figure 3.1 presents water level contours for December 19, 2007, and May 9, 2008, for the network of recording transducers installed for long-term water level monitoring at Barnes. The network on those dates included wells MW1D, MW2D, MW3D, MW4D, MW7, MW9, MW14D, MW15D, MW16D, and the Oentrich private well. The Oentrich well was removed from the network later on May 9, 2008, when the property owner replaced the pump and installed a new sanitary seal that limits access to the well (see Section 2.1).

Extensive documentation of the potentiometric surface at Barnes during the targeted investigation (Argonne 2008a) and subsequent monitoring events has indicated that operation of the public water supply wells strongly influences the groundwater flow direction. The accumulated data document a predominant direction of groundwater flow to the northeast under non-pumping conditions. In contrast, flow is toward the northwest, in the approximate direction of the public water supply wells, when pumping is occurring. These data (see also Figure 3.2) demonstrate that the public water supply wells are operated on a daily basis, with individual pumping and subsequent water level recovery periods that typically range from $3 \mathrm{hr}$ to $7 \mathrm{hr}$ in duration, resulting in groundwater levels (and apparent flow directions) that shift relatively continuously throughout much of each day. For this reason, water level data collected by the automatic recorders, which are coincident in time at all monitored locations, provide the primary basis for determination of the topology of the potentiometric surface at any point in the cycles of groundwater pumping and recovery. Water levels measured by hand over a finite time period in the areally distributed network of monitoring wells at this site cannot generally be relied on to yield a meaningful representation of groundwater flow directions, because of the short-term, transient nature of the documented water level variations that are related to pumping cycles in the public water supply wells.

The apparent flow directions illustrated for May 9, 2008 (Figure 3.1), were previously discussed (Argonne 2008d) as potentially anomalous, because of mechanical contouring of the 
available recorder data in the absence of values from monitoring well MW3D for the time intervals depicted. During the data downloads on July 9 and November 19, 2008, the recorders in MW3D were found to be damaged, and no data could be recovered. Previous findings have demonstrated that well MW3D and the Oentrich well represent critical locations for the interpretation of groundwater relationships at Barnes, because these two points largely constrain the southern and southwestern portions of the flow field in the vicinity of the carbon tetrachloride plume. The automatically recorded data from the remaining monitored wells suggest, however, that no significant changes have occurred in the relative patterns of groundwater levels at this site during the present review period.

The hydrographs in Figure 3.2 summarize data for the period January 1, 2008, to November 19, 2008, for the recording transducers. The hydrographs demonstrate that groundwater levels at Barnes have shown an overall increase of approximately $12 \mathrm{ft}$ during the period illustrated. A relatively stable period in July, August, and early September was followed by a fairly uniform increase in groundwater levels - by approximately $3 \mathrm{ft}$ - from mid September to mid November.

The water level data for well MW15D (Figure 3.2) indicate a persistent pattern of higher groundwater levels. This pattern of higher water levels in a deeper well might indicate that vertical hydraulic gradients are present.

The patterns in Figure 3.2 are similar to patterns recorded in 2007 during the targeted investigation and in subsequent monitoring events (Argonne 2008a-d). The hydrographs show responses to pumping of the public water supply wells that are characterized by drawdowns of as much as $2.25 \mathrm{ft}$ during pumping, with subsequent rebound when pumping stops. The most prominent drawdowns are consistently observed in wells MW1D and MW2D, which are the closest monitoring points to the public wells.

\subsection{Analytical Results for Volatile Organic Compounds in Groundwater Samples and Lateral Distribution of the Contaminants}

The analytical data for VOCs in the groundwater samples collected in October 2008 are in Table 3.2, together with data for the previous sampling events at Barnes. The lateral distribution of carbon tetrachloride in groundwater in the November 2007, March 2008, July 
2008, and October 2008 sampling events is illustrated in Figure 3.3; the distribution of chloroform during these four sampling events is illustrated in Figure 3.4.

Overall, the lateral distribution of carbon tetrachloride in groundwater in October 2008 is similar to the distribution during previous sampling events. The most significant changes during the current reporting period are the increase in the concentration of carbon tetrachloride in public well PWS2 (from $<1 \mu \mathrm{g} / \mathrm{L}$ in July to $1.7 \mu \mathrm{g} / \mathrm{L}$ in October) and a decrease at PWS3 (from $<1 \mu \mathrm{g} / \mathrm{L}$ in July to no detection in October).

The highest concentrations of carbon tetrachloride in sitewide monitoring have been found at well MW10S, which is located in the eastern portion of the former CCC/USDA facility and is screened at 93-103 ft below ground level (BGL). Concentrations at MW10S were in the range 49-68 $\mu \mathrm{g} / \mathrm{L}$ during the March, July, and October 2008 sampling events when the low-flow sampling method was used. Earlier, a significantly lower concentration of $11 \mu \mathrm{g} / \mathrm{L}$ was measured in MW10S in November 2007, when the three-well-volume purging method was used. Northwest of well MW10S (in the direction of flow toward the public wells when they are in operation), the carbon tetrachloride decreases to $18-20 \mu \mathrm{g} / \mathrm{L}$ at MW12M and MW13S. The recent sampling at sentinel well MW17 (located farther west, between MW13S and the two public wells) showed a slight increasing trend in carbon tetrachloride contamination, although all detections to date have been at trace levels. The evaluation of water level data and vertical contaminant distribution discussed in Section 3.3 indicates that wells MW10S, MW12M, and MW13S (where the highest carbon tetrachloride concentrations have been found) are screened in the intermediate aquifer zone.

The recent sampling at sentinel well MW1D, which is near MW13S, did not show the increasing trend suggested for intermediate-zone wells MW10S, MW12M, and MW13S. Well MW1D is installed in the deep aquifer zone (Section 3.3), to a depth similar to that of the public wells.

The lateral distribution of chloroform in groundwater in October 2008 is also similar to the distribution during previous sampling events. The highest concentration of chloroform in sitewide sampling has been found at well MW12M, which is located northwest of the former CCC/USDA facility and is screened at 90-100 ft BGL (in the intermediate aquifer zone), with concentrations of 2.6-5.1 $\mu \mathrm{g} / \mathrm{L}$. 


\subsection{Vertical Distribution of Carbon Tetrachloride and Evaluation of Data to Distinguish Aquifer Zones by Depth}

The designations "S," "M," and " $\mathrm{D}$ " in monitoring well names were assigned at the time of well installation. They indicate shallow, medium, and deep screens (relative depths) in an individual well, rather than the aquifer zone screened. After the March 2008 monitoring, a detailed evaluation of the hand-measured water levels (Table 3.1) and VOCs data (Table 3.2) was conducted in an attempt to differentiate aquifer zones by depth. The data collected in July 2008 and in October 2008 were evaluated similarly. The data from these events suggest at least three vertically distinct zones within the aquifer. The aquifer zones are as follows:

- Shallow Aquifer Zone. The only monitoring wells believed to be completed in the shallow zone are MW1S, MW11S, and MW12S. Wells MW1S and MW12S were dry during the March, July, and October 2008 sampling events. Well MW11S showed a water level elevation of approximately $1,312 \mathrm{ft}$ AMSL in the July event and approximately 1,314 ft AMSL in the October event.

- Intermediate Aquifer Zone. The intermediate zone is believed to be represented by monitoring wells MW10S, MW11M, MW12M, MW13S, and MW17. Water level elevations measured manually for these wells were approximately 1,255-1,258 ft AMSL in July 2008 and approximately 1,256-1,259 ft AMSL in October 2008. These wells were equipped with automatic water level recorders in November 2008, to obtain detailed data on the potential temporal variability of the hydraulic heads in this aquifer zone.

- Deep Aquifer Zone. Monitoring wells believed to represent the deep zone include MW1D, MW2D, MW3D, MW4D, MW5, MW6S, MW6D, MW7, MW8, MW9, MW10D, MW11D, MW12D, MW13D, MW14S, MW14D, MW15S, MW15D, MW16S, and MW16D. The water level elevations measured manually in these monitoring wells were approximately 1,229-1,239 ft AMSL in July 2008 and approximately 1,235-1,242 ft AMSL in October 2008. On the basis of its hand-measured water level in November 2007 (approximately 1,220 ft AMSL [Argonne 2008c] and the automatic measurements [Figure 3.2]), the Oentrich well is also considered to be screened in the deep aquifer zone. Wells MW2D, MW4D, MW7, MW9, 
MW14D, MW15D, and MW16D in the deep aquifer zone are presently equipped with water level recorders (as of November 2008). New recorders will be installed in wells MW1D and MW3D as soon as they are received from the manufacturer.

The vertical distribution of the carbon tetrachloride in groundwater indicates that the highest concentrations were detected in the intermediate zone, at wells MW10S $(68 \mu \mathrm{g} / \mathrm{L})$, MW12M (18 $\mu \mathrm{g} / \mathrm{L})$, and MW13S $(20 \mu \mathrm{g} / \mathrm{L})$. The deep-zone wells at these locations showed lower concentrations $(4.4 \mu / \mathrm{L}$ at MW10D, a trace [estimated at $0.9 \mu \mathrm{g} / \mathrm{L}$ ] at MW12D, and $6.6 \mu \mathrm{g} / \mathrm{L}$ at MW13D). Figures 3.5 and 3.6 illustrate the interpreted contaminant distributions in the intermediate and deep zones, respectively. The shallow-zone well (MW11S) continued to show no detectable concentrations of carbon tetrachloride (Table 3.2 and Figure 3.3). 
TABLE 3.1 Hand-measured water levels at Barnes, March-November 2008.

\begin{tabular}{|c|c|c|c|c|c|c|c|c|c|}
\hline \multirow[b]{3}{*}{ Well } & \multirow{3}{*}{$\begin{array}{l}\text { Reference } \\
\text { Elevation } \\
\text { (ft AMSL) }\end{array}$} & \multicolumn{8}{|c|}{ Water Level on Date Indicated } \\
\hline & & \multicolumn{2}{|c|}{$4 / 16 / 08$} & \multicolumn{2}{|c|}{$7 / 9-12 / 08$} & \multicolumn{2}{|c|}{$10 / 22-24 / 08$} & \multicolumn{2}{|c|}{$11 / 19 / 08$} \\
\hline & & $\mathrm{ft}$ TOC $^{\mathrm{a}}$ & $\mathrm{ft}$ AMSL & $\mathrm{ft} \mathrm{TOC}^{\mathrm{a}}$ & ft AMSL & $\mathrm{ft}$ TOC $^{\mathrm{a}}$ & $\mathrm{ft}$ AMSL & $\mathrm{ft}$ TOC $^{\mathrm{a}}$ & $\mathrm{ft}$ AMSL \\
\hline \multicolumn{10}{|c|}{ Shallow aquifer zone } \\
\hline MW1S & 1351.58 & Dry & - & Dry & - & Dry & - & Dry & - \\
\hline MW11S & 1336.58 & NM & - & 24.80 & 1311.78 & 22.50 & 1314.08 & NM & - \\
\hline MW12S & 1327.46 & Dry & - & Dry & - & Dry & - & Dry & - \\
\hline \multicolumn{10}{|c|}{ Intermediate aquifer zone } \\
\hline MW10S & 1331.33 & NM & - & 73.40 & 1257.93 & 72.00 & 1259.33 & 72.13 & 1259.20 \\
\hline MW11M & 1336.51 & NM & - & 78.85 & 1257.66 & 77.80 & 1258.71 & 77.4 & 1259.11 \\
\hline MW12M & 1327.46 & NM & - & 70.10 & 1257.36 & 69.72 & 1257.74 & 68.69 & 1258.77 \\
\hline MW13S & 1342.36 & NM & - & 87.00 & 1255.36 & 86.00 & 1256.36 & 85.02 & 1257.34 \\
\hline MW17 & 1351.77 & NM & - & 96.60 & 1255.17 & 95.15 & 1256.62 & 94.81 & 1256.96 \\
\hline \multicolumn{10}{|c|}{ Deep aquifer zone } \\
\hline MW1D & 1351.33 & 123.63 & 1227.70 & 119.40 & 1231.93 & 113.77 & 1237.56 & 113.62 & 1237.71 \\
\hline MW2D & 1348.85 & 121.4 & 1227.45 & 117.15 & 1231.70 & 113.55 & 1235.30 & 111.61 & 1237.24 \\
\hline MW3D & 1345.99 & 118.03 & 1227.96 & 113.30 & 1232.69 & 108.50 & 1237.49 & 108.2 & 1237.79 \\
\hline MW4D & 1326.32 & 100.07 & 1226.25 & 93.60 & 1232.72 & 89.90 & 1236.42 & 90.72 & 1235.60 \\
\hline MW5 & 1327.20 & NM & - & 93.80 & 1233.40 & 91.40 & 1235.80 & NM & - \\
\hline MW6S & 1323.13 & NM & - & 88.10 & 1235.03 & 84.60 & 1238.53 & NM & - \\
\hline MW6D & 1323.15 & NM & - & 89.50 & 1233.65 & 87.15 & 1236.00 & NM & - \\
\hline MW7 & 1329.91 & 103.81 & 1226.10 & 97.50 & 1232.41 & 94.90 & 1235.01 & 96.67 & 1233.24 \\
\hline MW8 & 1330.06 & NM & - & 95.75 & 1234.31 & 93.40 & 1236.66 & NM & - \\
\hline MW9 & 1321.86 & 94.56 & 1227.30 & 87.65 & 1234.21 & 86.60 & 1235.26 & 86.19 & 1235.67 \\
\hline MW10D & 1331.33 & NM & - & 97.30 & 1234.03 & 95.00 & 1236.33 & NM & - \\
\hline MW11D & 1336.53 & NM & - & 102.10 & 1234.43 & 101.03 & 1235.50 & NM & - \\
\hline MW12D & 1327.52 & NM & - & 93.70 & 1233.82 & 91.12 & 1236.40 & NM & - \\
\hline MW13D & 1342.37 & NM & - & 107.90 & 1234.47 & 105.30 & 1237.07 & NM & - \\
\hline MW14S & 1332.69 & NM & - & 99.40 & 1233.29 & 96.20 & 1236.49 & NM & - \\
\hline MW14D & 1332.74 & 105.84 & 1226.90 & 101.00 & 1231.74 & 96.10 & 1236.64 & 96.18 & 1236.56 \\
\hline MW15S & 1309.34 & NM & - & 80.30 & 1229.04 & 73.20 & 1236.14 & NM & - \\
\hline MW15D & 1309.29 & 78.25 & 1231.04 & 70.30 & 1238.99 & 67.60 & 1241.69 & 67.33 & 1241.96 \\
\hline MW16S & 1299.47 & NM & - & 67.35 & 1232.12 & 64.80 & 1234.67 & NM & - \\
\hline MW16D & 1299.52 & 73.05 & 1226.47 & 66.30 & 1233.22 & 63.90 & 1235.62 & 64.31 & 1235.21 \\
\hline Oentrich $^{b}$ & 1336.93 & 114.97 & 1221.96 & NM & NM & NM & - & NM & - \\
\hline
\end{tabular}

a TOC, top of casing.

b The Oentrich well water level was measured from the concrete at the top of the well vault. The value shown was corrected by $5.5 \mathrm{ft}$ to give a measured depth from the top of the casing.

c NM, not measured (sampled from faucet). 
TABLE 3.2 Analytical results from the AGEM Laboratory for volatile organic compounds in groundwater samples collected at Barnes, July 2006 to October 2008.

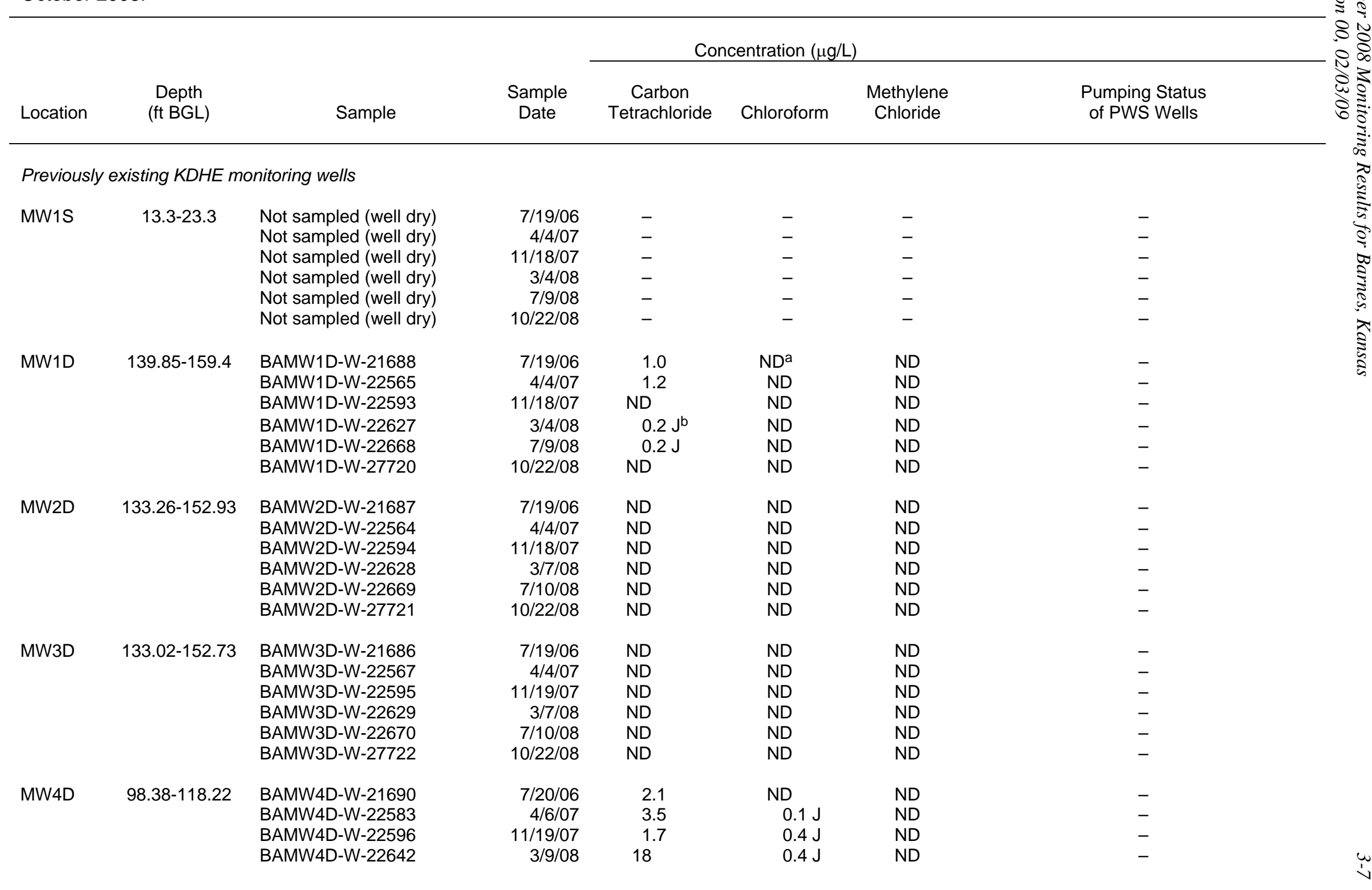


TABLE 3.2 (Cont.)

\begin{tabular}{|c|c|c|c|c|c|c|c|}
\hline \multirow[b]{2}{*}{ Location } & \multirow[b]{2}{*}{$\begin{array}{l}\text { Depth } \\
\text { (ft BGL) }\end{array}$} & \multirow[b]{2}{*}{ Sample } & \multirow[b]{2}{*}{$\begin{array}{l}\text { Sample } \\
\text { Date }\end{array}$} & \multicolumn{3}{|c|}{ Concentration $(\mu \mathrm{g} / \mathrm{L})$} & \multirow[b]{2}{*}{$\begin{array}{l}\text { Pumping Status } \\
\text { of PWS Wells }\end{array}$} \\
\hline & & & & $\begin{array}{c}\text { Carbon } \\
\text { Tetrachloride }\end{array}$ & Chloroform & $\begin{array}{l}\text { Methylene } \\
\text { Chloride }\end{array}$ & \\
\hline \multicolumn{8}{|c|}{ Previously existing KDHE monitoring wells (cont.) } \\
\hline \multirow[t]{2}{*}{ MW4D } & $98.38-118.22$ & BAMW4D-W-22671 & $7 / 12 / 08$ & 9.4 & $0.5 \mathrm{~J}$ & ND & - \\
\hline & & BAMW4D-W-27723 & 10/23/08 & 7.6 & ND & ND & - \\
\hline \multicolumn{8}{|c|}{ CCC/USDA wells installed during the 2006-2007 investigation } \\
\hline \multirow[t]{5}{*}{ MW5 } & $110-120$ & BAMW5-W-22589 & $4 / 6 / 07$ & $0.6 \mathrm{~J}$ & ND & ND & - \\
\hline & & BAMW5-W-22597 & 11/19/07 & $0.6 \mathrm{~J}$ & ND & ND & - \\
\hline & & BAMW5-W-22637 & 3/8/08 & $0.7 \mathrm{~J}$ & ND & ND & - \\
\hline & & BAMW5-W-22672 & 7/11/08 & ND & ND & ND & - \\
\hline & & BAMW5-W-27724 & 10/23/08 & 3.0 & ND & ND & - \\
\hline \multirow[t]{5}{*}{ MW6S } & $90.5-100.5$ & Not sampled (well dry) & $4 / 4 / 07$ & - & - & - & - \\
\hline & & BAMW6S-W-22598 & $11 / 19 / 07$ & $0.3 \mathrm{~J}$ & ND & ND & - \\
\hline & & BAMW6S-W-22635 & $3 / 8 / 08$ & $0.4 \mathrm{~J}$ & ND & ND & - \\
\hline & & BAMW6S-W-22673 & 7/11/08 & ND & ND & ND & - \\
\hline & & BAMW6S-W-27725 & $10 / 23 / 08$ & ND & ND & ND & - \\
\hline \multirow[t]{5}{*}{ MW6D } & $105-115$ & BAMW6D-W-22573 & $4 / 5 / 07$ & ND & ND & ND & - \\
\hline & & BAMW6D-W-22599 & $11 / 19 / 07$ & $0.5 \mathrm{~J}$ & ND & ND & - \\
\hline & & BAMW6D-W-22636 & $3 / 8 / 08$ & $0.8 \mathrm{~J}$ & ND & ND & - \\
\hline & & BAMW6D-W-22674 & $7 / 11 / 08$ & $0.9 \mathrm{~J}$ & ND & ND & - \\
\hline & & BAMW6D-W-27726 & $10 / 23 / 08$ & 1.1 & ND & ND & - \\
\hline \multirow[t]{5}{*}{ MW7 } & $116-126$ & BAMW7-W-22588 & $4 / 6 / 07$ & 1.0 & ND & ND & - \\
\hline & & BAMW7-W-22600 & 11/19/07 & 2.6 & ND & ND & - \\
\hline & & BAMW7-W-22643 & 3/9/08 & 2.8 & ND & ND & - \\
\hline & & BAMW7-W-22675 & 7/12/08 & 1.7 & ND & ND & - \\
\hline & & BAMW7-W-27727 & $10 / 23 / 08$ & 2.1 & ND & ND & - \\
\hline \multirow[t]{5}{*}{ MW8 } & $110-120$ & BAMW8-W-22584 & $4 / 6 / 07$ & 14 & $0.7 \mathrm{~J}$ & ND & - \\
\hline & & BAMW8-W-22601 & 11/19/07 & 23 & $0.6 \mathrm{~J}$ & ND & - \\
\hline & & BAMW8-W-22652 & $3 / 10 / 08$ & 19 & $0.6 \mathrm{~J}$ & ND & - \\
\hline & & BAMW8-W-22676 & 7/11/08 & 21 & $0.6 \mathrm{~J}$ & ND & - \\
\hline & & BAMW8-W-27728 & 10/23/08 & 24 & 1.0 & ND & - \\
\hline
\end{tabular}


TABLE 3.2 (Cont.)

\begin{tabular}{|c|c|c|c|c|c|c|c|}
\hline \multirow[b]{2}{*}{ Location } & \multirow[b]{2}{*}{$\begin{array}{l}\text { Depth } \\
\text { (ft BGL) }\end{array}$} & \multirow[b]{2}{*}{ Sample } & \multirow[b]{2}{*}{$\begin{array}{l}\text { Sample } \\
\text { Date }\end{array}$} & \multicolumn{3}{|c|}{ Concentration $(\mu \mathrm{g} / \mathrm{L})$} & \multirow[b]{2}{*}{$\begin{array}{l}\text { Pumping Status } \\
\text { of PWS Wells }\end{array}$} \\
\hline & & & & $\begin{array}{c}\text { Carbon } \\
\text { Tetrachloride }\end{array}$ & Chloroform & $\begin{array}{l}\text { Methylene } \\
\text { Chloride }\end{array}$ & \\
\hline \multicolumn{8}{|c|}{ CCC/USDA wells installed during the 2006-2007 investigation (cont.) } \\
\hline \multirow[t]{5}{*}{ MW9 } & $100-110$ & BAMW9-W-22582 & $4 / 5 / 07$ & 1.0 & ND & ND & - \\
\hline & & BAMW9-W-22602 & 11/19/07 & 7.7 & $0.6 \mathrm{~J}$ & ND & - \\
\hline & & BAMW9-W-22647 & $3 / 9 / 08$ & 3.0 & $0.3 \mathrm{~J}$ & ND & - \\
\hline & & BAMW9-W-22678 & $7 / 11 / 08$ & 1.3 & $0.3 \mathrm{~J}$ & ND & - \\
\hline & & BAMW9-W-27729 & $10 / 24 / 08$ & 2.2 & $0.2 \mathrm{~J}$ & ND & - \\
\hline \multirow[t]{5}{*}{ MW10S } & $93-103$ & BAMW10S-W-22586 & $4 / 6 / 07$ & 20 & 1.4 & ND & - \\
\hline & & BAMW10S-W-22603 & $11 / 19 / 07$ & 11 & $0.7 \mathrm{~J}$ & ND & - \\
\hline & & BAMW10S-W-22649 & 3/10/08 & 56 & 2.0 & ND & - \\
\hline & & BAMW10S-W-22679 & $7 / 11 / 08$ & 49 & 1.8 & ND & - \\
\hline & & BAMW10S-W-27730 & $10 / 23 / 08$ & 68 & 2.3 & ND & - \\
\hline \multirow[t]{5}{*}{ MW10D } & $115-125$ & BAMW10D-W-22585 & $4 / 6 / 07$ & 2.4 & $0.2 \mathrm{~J}$ & ND & - \\
\hline & & BAMW10D-W-22604 & $11 / 19 / 07$ & 6.3 & $0.5 \mathrm{~J}$ & ND & - \\
\hline & & BAMW10D-W-22646 & $3 / 9 / 08$ & 5.7 & $0.5 \mathrm{~J}$ & ND & - \\
\hline & & BAMW10D-W-22680 & $7 / 11 / 08$ & 3.9 & $0.7 \mathrm{~J}$ & ND & - \\
\hline & & BAMW10D-W-27731 & $10 / 23 / 08$ & 4.4 & $0.6 \mathrm{~J}$ & ND & - \\
\hline \multirow[t]{5}{*}{ MW11S } & $40-50$ & BAMW11S-W-22570 & $4 / 4 / 07$ & ND & 1.1 & ND & - \\
\hline & & BAMW11S-W-22605 & $11 / 19 / 07$ & ND & $0.6 \mathrm{~J}$ & ND & - \\
\hline & & BAMW11S-W-22630 & $3 / 5 / 08$ & ND & $0.6 \mathrm{~J}$ & ND & - \\
\hline & & BAMW11S-W-22681 & 7/10/08 & ND & $0.4 \mathrm{~J}$ & ND & - \\
\hline & & BAMW11S-W-27732 & $10 / 23 / 08$ & ND & $0.3 \mathrm{~J}$ & ND & - \\
\hline \multirow[t]{5}{*}{ MW11M } & $90-100$ & BAMW11M-W-22572 & $4 / 5 / 07$ & ND & ND & ND & - \\
\hline & & BAMW11M-W-22606 & $11 / 19 / 07$ & 3.7 & ND & ND & - \\
\hline & & BAMW11M-W-22644 & $3 / 6 / 08$ & 2.4 & $0.5 \mathrm{~J}$ & ND & - \\
\hline & & BAMW11M-W-22682 & $7 / 10 / 08$ & 2.4 & $0.7 \mathrm{~J}$ & ND & - \\
\hline & & BAMW11M-W-27733 & 10/23/08 & 1.7 & 2.1 & ND & - \\
\hline \multirow[t]{3}{*}{ MW11D } & $125-135$ & BAMW11D-W-22571 & $4 / 4 / 07$ & 1.1 & ND & ND & - \\
\hline & & BAMW11D-W-22607 & $11 / 19 / 07$ & $0.8 \mathrm{~J}$ & ND & ND & - \\
\hline & & BAMW11D-W-22639 & $3 / 5 / 08$ & $0.4 \mathrm{~J}$ & ND & ND & - \\
\hline
\end{tabular}


TABLE 3.2 (Cont.)

\begin{tabular}{|c|c|c|c|c|c|c|c|}
\hline \multirow[b]{2}{*}{ Location } & \multirow[b]{2}{*}{$\begin{array}{c}\text { Depth } \\
\text { (ft BGL) }\end{array}$} & \multirow[b]{2}{*}{ Sample } & \multirow[b]{2}{*}{$\begin{array}{l}\text { Sample } \\
\text { Date }\end{array}$} & \multicolumn{3}{|c|}{ Concentration $(\mu \mathrm{g} / \mathrm{L})$} & \multirow[b]{2}{*}{$\begin{array}{l}\text { Pumping Status } \\
\text { of PWS Wells }\end{array}$} \\
\hline & & & & $\begin{array}{l}\text { Carbon } \\
\text { Tetrachloride }\end{array}$ & Chloroform & $\begin{array}{l}\text { Methylene } \\
\text { Chloride }\end{array}$ & \\
\hline \multicolumn{8}{|c|}{ CCC/USDA wells installed during the 2006-2007 investigation (cont.) } \\
\hline \multirow[t]{2}{*}{ MW11D } & $125-135$ & BAMW11D-W-22683 & $7 / 10 / 08$ & $0.9 \mathrm{~J}$ & ND & ND & - \\
\hline & & BAMW11D-W-27734 & $10 / 23 / 08$ & $0.9 \mathrm{~J}$ & $0.2 \mathrm{~J}$ & ND & - \\
\hline \multirow[t]{5}{*}{ MW12S } & $43-50$ & Not sampled (well dry) & $4 / 5 / 07$ & - & - & - & - \\
\hline & & Not sampled (well dry) & $11 / 19 / 07$ & - & - & - & - \\
\hline & & Not sampled (well dry) & $3 / 10 / 08$ & - & - & - & - \\
\hline & & Not sampled (well dry) & $7 / 10 / 08$ & - & - & - & - \\
\hline & & Not sampled (well dry) & $10 / 22 / 08$ & - & - & - & - \\
\hline \multirow[t]{5}{*}{ MW12M } & $90-100$ & BAMW12M-W-22580 & $4 / 5 / 07$ & 20 & 4.2 & ND & - \\
\hline & & BAMW12M-W-22609 & $11 / 19 / 07$ & 18 & 5.1 & ND & - \\
\hline & & BAMW12M-W-22651 & $3 / 10 / 08$ & 18 & 2.6 & ND & - \\
\hline & & BAMW12M-W-22685 & $7 / 10 / 08$ & 27 & 4.2 & ND & - \\
\hline & & BAMW12M-W-27736 & $10 / 22 / 08$ & 18 & 4.5 & ND & - \\
\hline \multirow[t]{5}{*}{ MW12D } & $115-125$ & BAMW12D-W-22576 & $4 / 5 / 07$ & $0.6 \mathrm{~J}$ & ND & ND & - \\
\hline & & BAMW12D-W-22610 & $11 / 18 / 07$ & 1.6 & ND & ND & - \\
\hline & & BAMW12D-W-22641 & $3 / 9 / 08$ & 1.0 & ND & ND & - \\
\hline & & BAMW12D-W-22686 & $7 / 11 / 08$ & $0.7 \mathrm{~J}$ & ND & ND & - \\
\hline & & BAMW12D-W-27737 & $10 / 22 / 08$ & $0.9 \mathrm{~J}$ & ND & ND & - \\
\hline \multirow[t]{5}{*}{ MW13S } & $112-122$ & BAMW13S-W-22575 & $4 / 5 / 07$ & 21 & 1.6 & ND & - \\
\hline & & BAMW13S-W-22611 & $11 / 19 / 07$ & 17 & 1.8 & ND & - \\
\hline & & BAMW13S-W-22650 & $3 / 10 / 08$ & 17 & 1.5 & ND & - \\
\hline & & BAMW13S-W-22687 & 7/9/08 & 17 & 1.9 & ND & - \\
\hline & & BAMW13S-W-27738 & $10 / 22 / 08$ & 20 & 1.6 & ND & - \\
\hline \multirow[t]{5}{*}{ MW13D } & $127-137$ & BAMW13D-W-22574 & $4 / 5 / 07$ & 3.5 & $0.4 \mathrm{~J}$ & ND & - \\
\hline & & BAMW13D-W-22612 & $11 / 19 / 07$ & 5.9 & $0.2 \mathrm{~J}$ & ND & - \\
\hline & & BAMW13D-W-22645 & $3 / 9 / 08$ & 11 & 1.1 & ND & - \\
\hline & & BAMW13D-W-22688 & $7 / 9 / 08$ & 5.9 & $0.9 \mathrm{~J}$ & ND & - \\
\hline & & BAMW13D-W-27739 & $10 / 22 / 08$ & 6.6 & $0.6 \mathrm{~J}$ & ND & - \\
\hline
\end{tabular}


TABLE 3.2 (Cont.)

\begin{tabular}{|c|c|c|c|c|c|c|c|}
\hline \multirow[b]{2}{*}{ Location } & \multirow[b]{2}{*}{$\begin{array}{c}\text { Depth } \\
\text { (ft BGL) }\end{array}$} & \multirow[b]{2}{*}{ Sample } & \multirow[b]{2}{*}{$\begin{array}{l}\text { Sample } \\
\text { Date }\end{array}$} & \multicolumn{3}{|c|}{ Concentration $(\mu \mathrm{g} / \mathrm{L})$} & \multirow[b]{2}{*}{$\begin{array}{l}\text { Pumping Status } \\
\text { of PWS Wells }\end{array}$} \\
\hline & & & & $\begin{array}{c}\text { Carbon } \\
\text { Tetrachloride }\end{array}$ & Chloroform & $\begin{array}{l}\text { Methylene } \\
\text { Chloride }\end{array}$ & \\
\hline \multicolumn{8}{|c|}{ CCC/USDA wells installed during the 2006-2007 investigation (cont.) } \\
\hline \multirow[t]{5}{*}{ MW14S } & $108-118$ & BAMW14S-W-22569 & $4 / 4 / 07$ & $0.9 \mathrm{~J}$ & ND & ND & - \\
\hline & & BAMW14S-W-22613 & $11 / 18 / 07$ & 1.2 & ND & ND & - \\
\hline & & BAMW14S-W-22640 & $3 / 8 / 08$ & 4.3 & $0.3 \mathrm{~J}$ & ND & - \\
\hline & & BAMW14S-W-22689 & $7 / 10 / 08$ & 5.6 & $0.3 \mathrm{~J}$ & ND & - \\
\hline & & BAMW14S-W-27740 & $10 / 22 / 08$ & 5.6 & $0.3 \mathrm{~J}$ & ND & - \\
\hline \multirow[t]{5}{*}{ MW14D } & $123-133$ & BAMW14D-W-22568 & $4 / 4 / 07$ & 1.2 & ND & ND & - \\
\hline & & BAMW14D-W-22614 & $11 / 18 / 07$ & $0.6 \mathrm{~J}$ & ND & ND & - \\
\hline & & BAMW14D-W-22638 & $3 / 8 / 08$ & $0.7 \mathrm{~J}$ & ND & ND & - \\
\hline & & BAMW14D-W-22690 & 7/10/08 & $0.5 \mathrm{~J}$ & ND & ND & - \\
\hline & & BAMW14D-W-27741 & $10 / 22 / 08$ & ND & ND & ND & - \\
\hline \multirow[t]{5}{*}{ MW15S } & $88-98$ & BAMW15S-W-22560 & $4 / 4 / 07$ & 1.5 & ND & ND & - \\
\hline & & BAMW15S-W-22615 & $11 / 18 / 07$ & 8.7 & $0.4 \mathrm{~J}$ & ND & - \\
\hline & & BAMW15S-W-22648 & $3 / 10 / 08$ & 1.8 & $0.2 \mathrm{~J}$ & ND & - \\
\hline & & BAMW15S-W-22691 & $7 / 12 / 08$ & 2.2 & $0.3 \mathrm{~J}$ & ND & - \\
\hline & & BAMW15S-W-27742 & $10 / 23 / 08$ & 1.9 & ND & ND & - \\
\hline \multirow[t]{5}{*}{ MW15D } & $105-115$ & BAMW15D-W-22561 & $4 / 4 / 07$ & ND & ND & ND & - \\
\hline & & BAMW15D-W-22616 & $11 / 18 / 07$ & ND & ND & ND & - \\
\hline & & BAMW15D-W-22631 & $3 / 8 / 08$ & $0.2 \mathrm{~J}$ & ND & ND & - \\
\hline & & BAMW15D-W-22692 & $7 / 12 / 08$ & ND & ND & ND & - \\
\hline & & BAMW15D-W-27743 & $10 / 24 / 08$ & ND & ND & ND & - \\
\hline \multirow{5}{*}{ MW16S } & $76-86$ & BAMW16S-W-22563 & $4 / 4 / 07$ & ND & ND & ND & - \\
\hline & & BAMW16S-W-22617 & $11 / 19 / 07$ & ND & ND & ND & - \\
\hline & & BAMW16S-W-22632 & $3 / 7 / 08$ & $0.4 \mathrm{~J}$ & ND & ND & - \\
\hline & & BAMW16S-W-22693 & 7/11/08 & ND & ND & ND & - \\
\hline & & BAMW16S-W-27744 & $10 / 23 / 08$ & $0.9 \mathrm{~J}$ & ND & ND & - \\
\hline \multirow[t]{3}{*}{ MW16D } & $90-100$ & BAMW16D-W-22562 & $4 / 4 / 07$ & ND & ND & ND & - \\
\hline & & BAMW16D-W-22618 & $11 / 19 / 07$ & ND & ND & ND & - \\
\hline & & BAMW16D-W-22633 & $3 / 7 / 08$ & ND & ND & ND & - \\
\hline
\end{tabular}


TABLE 3.2 (Cont.)

\begin{tabular}{|c|c|c|c|c|c|c|c|}
\hline \multirow[b]{2}{*}{ Location } & \multirow[b]{2}{*}{$\begin{array}{c}\text { Depth } \\
\text { (ft BGL) }\end{array}$} & \multirow[b]{2}{*}{ Sample } & \multirow[b]{2}{*}{$\begin{array}{l}\text { Sample } \\
\text { Date }\end{array}$} & \multicolumn{3}{|c|}{ Concentration $(\mu \mathrm{g} / \mathrm{L})$} & \multirow[b]{2}{*}{$\begin{array}{l}\text { Pumping Status } \\
\text { of PWS Wells }\end{array}$} \\
\hline & & & & $\begin{array}{l}\text { Carbon } \\
\text { Tetrachloride }\end{array}$ & Chloroform & $\begin{array}{l}\text { Methylene } \\
\text { Chloride }\end{array}$ & \\
\hline \multicolumn{8}{|c|}{ CCC/USDA wells installed during the 2006-2007 investigation (cont.) } \\
\hline \multirow[t]{2}{*}{ MW16D } & $90-100$ & BAMW16D-W-22694 & $7 / 11 / 08$ & ND & ND & ND & - \\
\hline & & BAMW16D-W-27745 & $10 / 23 / 08$ & ND & ND & ND & - \\
\hline \multirow[t]{5}{*}{ MW17 } & $120-130$ & BAMW17D-W-22566 & $4 / 4 / 07$ & ND & ND & ND & - \\
\hline & & BAMW17D-W-22619 & $11 / 19 / 07$ & ND & ND & ND & - \\
\hline & & BAMW17-W-22634 & $3 / 5 / 08$ & $0.3 \mathrm{~J}$ & ND & ND & - \\
\hline & & BAMW17-W-22695 & $7 / 9 / 08$ & $0.4 \mathrm{~J}$ & ND & ND & - \\
\hline & & BAMW17-W-27746 & $10 / 22 / 08$ & $0.7 \mathrm{~J}$ & ND & ND & - \\
\hline \multicolumn{8}{|c|}{ Private wells } \\
\hline \multirow[t]{7}{*}{ Oentrich } & 150 & BAOENT-W-21693 & $7 / 20 / 06$ & $0.3 \mathrm{~J}$ & ND & ND & - \\
\hline & & BAOENT-W-21713 & $8 / 2 / 06$ & $0.6 \mathrm{~J}$ & ND & ND & - \\
\hline & & BAOENTRICH-W-22579 & $4 / 5 / 07$ & $0.6 \mathrm{~J}$ & ND & ND & - \\
\hline & & BAOENTRICH-W-22622 & $11 / 19 / 07$ & $0.8 \mathrm{~J}$ & ND & ND & - \\
\hline & & BAOENTRICH-W-22654 & $3 / 6 / 08$ & 1.3 & ND & ND & - \\
\hline & & BAOENTRICH-W-22695 & $7 / 11 / 08$ & $0.3 \mathrm{~J}$ & ND & ND & - \\
\hline & & BAOENTRICH-W-27747 & $10 / 23 / 08$ & $0.9 \mathrm{~J}$ & ND & ND & - \\
\hline \multirow[t]{2}{*}{ Sedivy } & 138 & BACW-W-21849 & $8 / 22 / 06$ & ND & ND & ND & - \\
\hline & & BASED2-W-21913 & $9 / 13 / 06$ & ND & ND & ND & - \\
\hline Sedivy1 & 90 & Not sampled (well dry) & $9 / 13 / 06$ & - & - & - & - \\
\hline \multicolumn{8}{|c|}{ Public water supply wells } \\
\hline \multirow[t]{8}{*}{ PWS2 } & 155 & BAPWS2-W-22510 & 3/9/07 & ND & ND & ND & Well has been pumping today. \\
\hline & & BAPW2-W-22578 & $4 / 5 / 07$ & ND & ND & ND & Sampled after pump had run for $5-10$ in. \\
\hline & & BAPW2-W-22620 & $11 / 20 / 07$ & ND & ND & ND & Well on at time of sampling. \\
\hline & & BAPWS2-W-22655 & $3 / 6 / 08$ & ND & ND & ND & sampled. \\
\hline & & BAPWS2-W-22696 & $7 / 11 / 08$ & $0.8 \mathrm{~J}$ & ND & ND & Running for $30 \mathrm{~min}$. \\
\hline & & - & $7 / 14 / 08^{c}$ & 1.3 & - & - & Unknown. \\
\hline & & - & $8 / 3 / 08^{c}$ & 0.89 & - & - & Unkknown \\
\hline & & BAPW2-W-27748 & $10 / 23 / 08$ & 1.7 & ND & ND & Well was pumping for $5 \mathrm{~min}$. \\
\hline
\end{tabular}




\begin{tabular}{|c|c|c|c|c|c|c|c|}
\hline \multirow[b]{2}{*}{ Location } & \multirow[b]{2}{*}{$\begin{array}{l}\text { Depth } \\
\text { (ft BGL) }\end{array}$} & \multirow[b]{2}{*}{ Sample } & \multirow[b]{2}{*}{$\begin{array}{l}\text { Sample } \\
\text { Date }\end{array}$} & \multicolumn{3}{|c|}{ Concentration $(\mu \mathrm{g} / \mathrm{L})$} & \multirow[b]{2}{*}{$\begin{array}{l}\text { Pumping Status } \\
\text { of PWS Wells }\end{array}$} \\
\hline & & & & $\begin{array}{l}\text { Carbon } \\
\text { Tetrachloride }\end{array}$ & Chloroform & $\begin{array}{l}\text { Methylene } \\
\text { Chloride }\end{array}$ & \\
\hline \multicolumn{8}{|c|}{ Public water supply wells (cont.) } \\
\hline \multirow[t]{7}{*}{ PWS3 } & 160 & BAPWS3-W-22511 & $3 / 9 / 07$ & $0.2 \mathrm{~J}$ & ND & ND & Well has been pumping today. \\
\hline & & BAPW3-W-22577 & $4 / 5 / 07$ & ND & ND & ND & Well has been pumping all day. \\
\hline & & BAPW3-W-22621 & $11 / 20 / 07$ & ND & ND & ND & Well on at time of sampling. \\
\hline & & BAPWS3-W-22656 & $3 / 6 / 08$ & ND & ND & ND & $\begin{array}{l}\text { Sample collected from tap in well house. } \\
\text { Let water run from tap for } 2-3 \text { min, } \\
\text { then sampled. }\end{array}$ \\
\hline & & BAPWS3-W-22697 & 7/11/08 & $0.2 \mathrm{~J}$ & ND & ND & Running for $30 \mathrm{~min}$ \\
\hline & & - & $7 / 14 / 08^{c}$ & ND & - & - & Unknown. \\
\hline & & BAPW3-W-27749 & $10 / 23 / 08$ & ND & ND & ND & Well was pumping for 30 min. \\
\hline
\end{tabular}

a ND, contaminant not detected at an instrument detection limit of $0.1 \mu \mathrm{g} / \mathrm{L}$.

b Qualifier J indicates an estimated concentration below the purge-and-trap method quantitation limit of $1.0 \mu \mathrm{g} / \mathrm{L}$.

c Sample collected by City of Barnes. Complete results of the city's sampling in 1986-2008 are in Appendix E. 

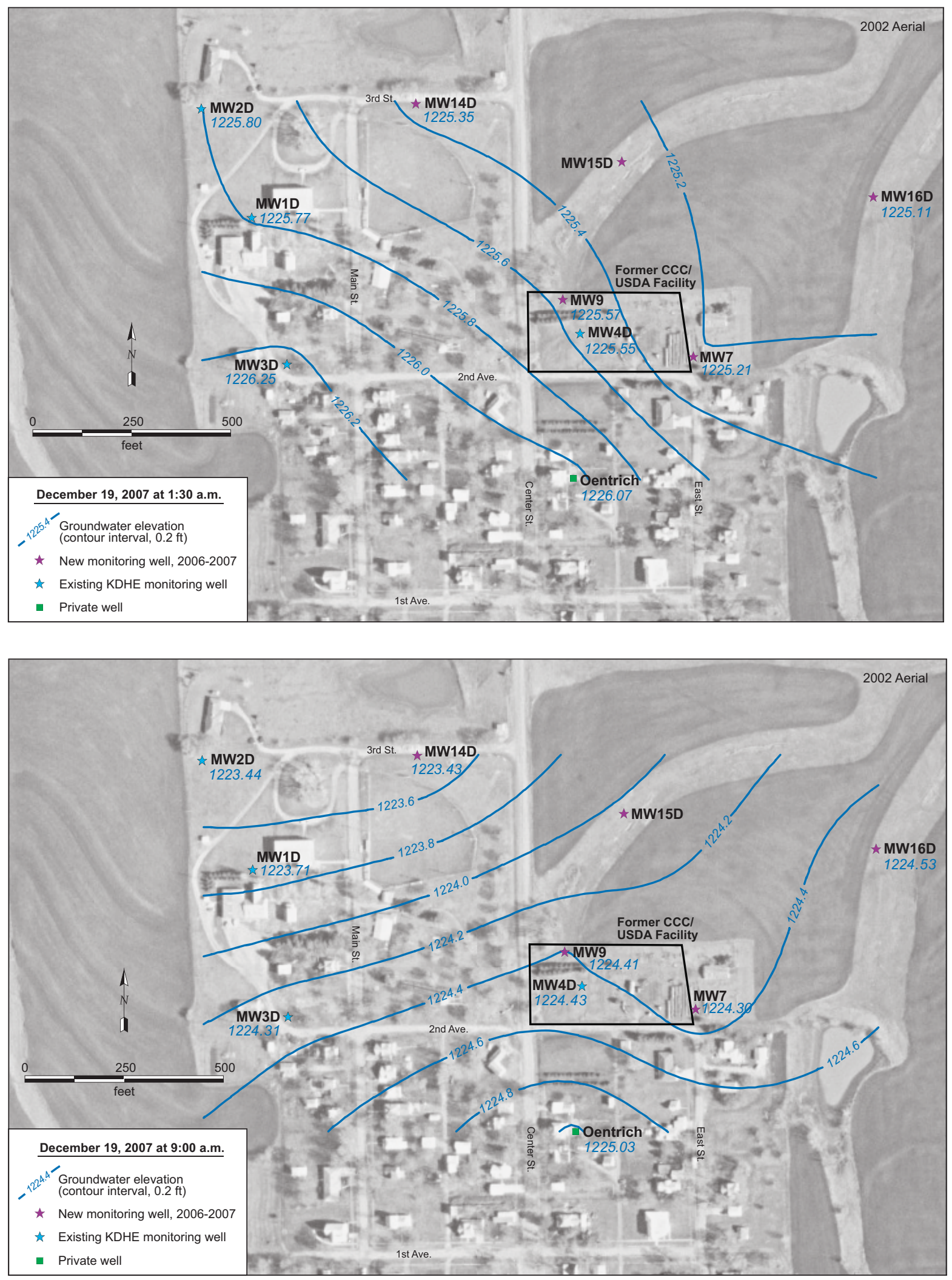

FIGURE 3.1 Potentiometric surface maps depicting the groundwater flow direction at Barnes in December 2007 and May 2008, before pumping of the public water supply wells (top panels) and during pumping (bottom panels). Source of photograph: NAPP (2002). 

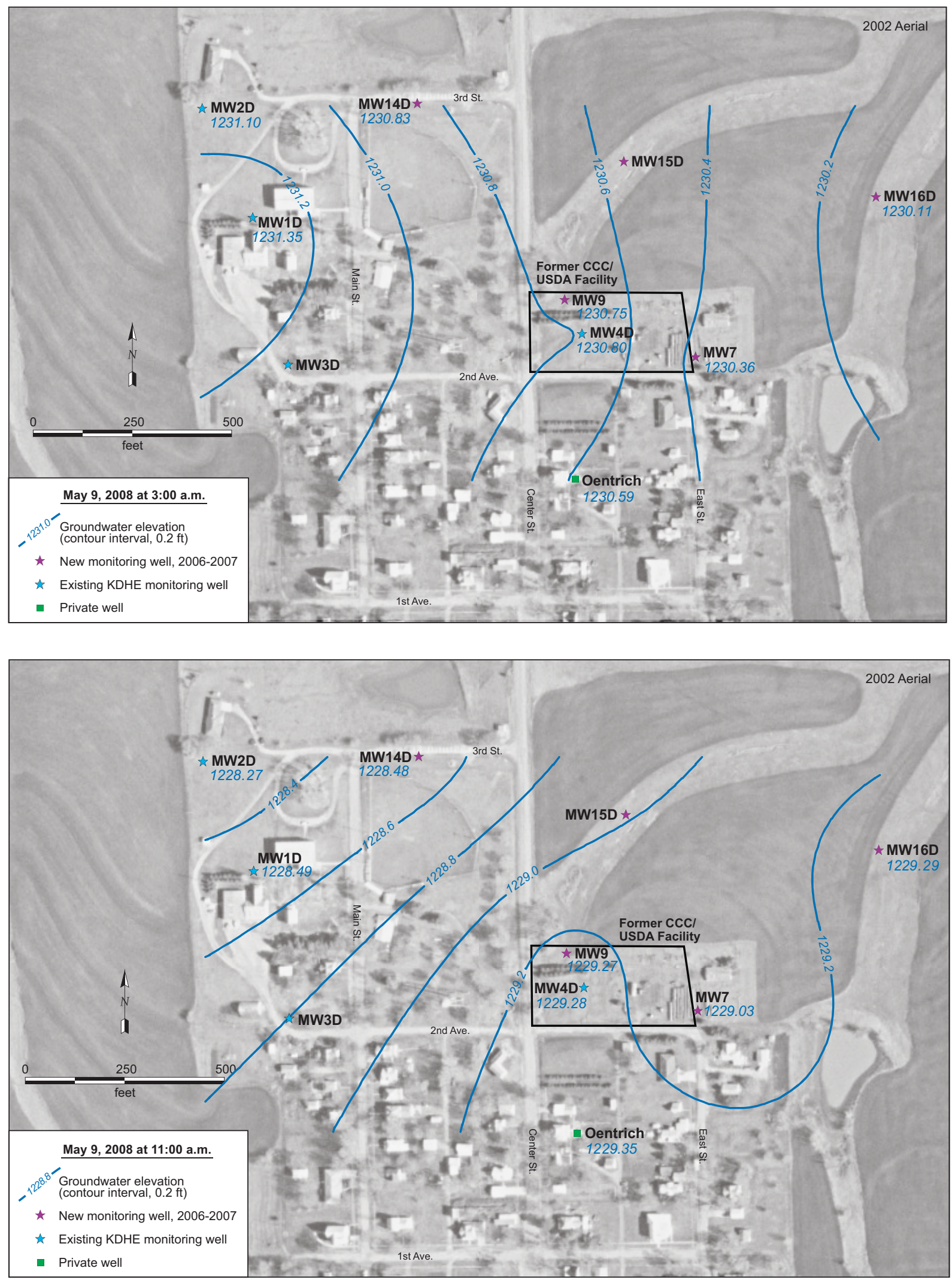

FIGURE 3.1 (cont.) 

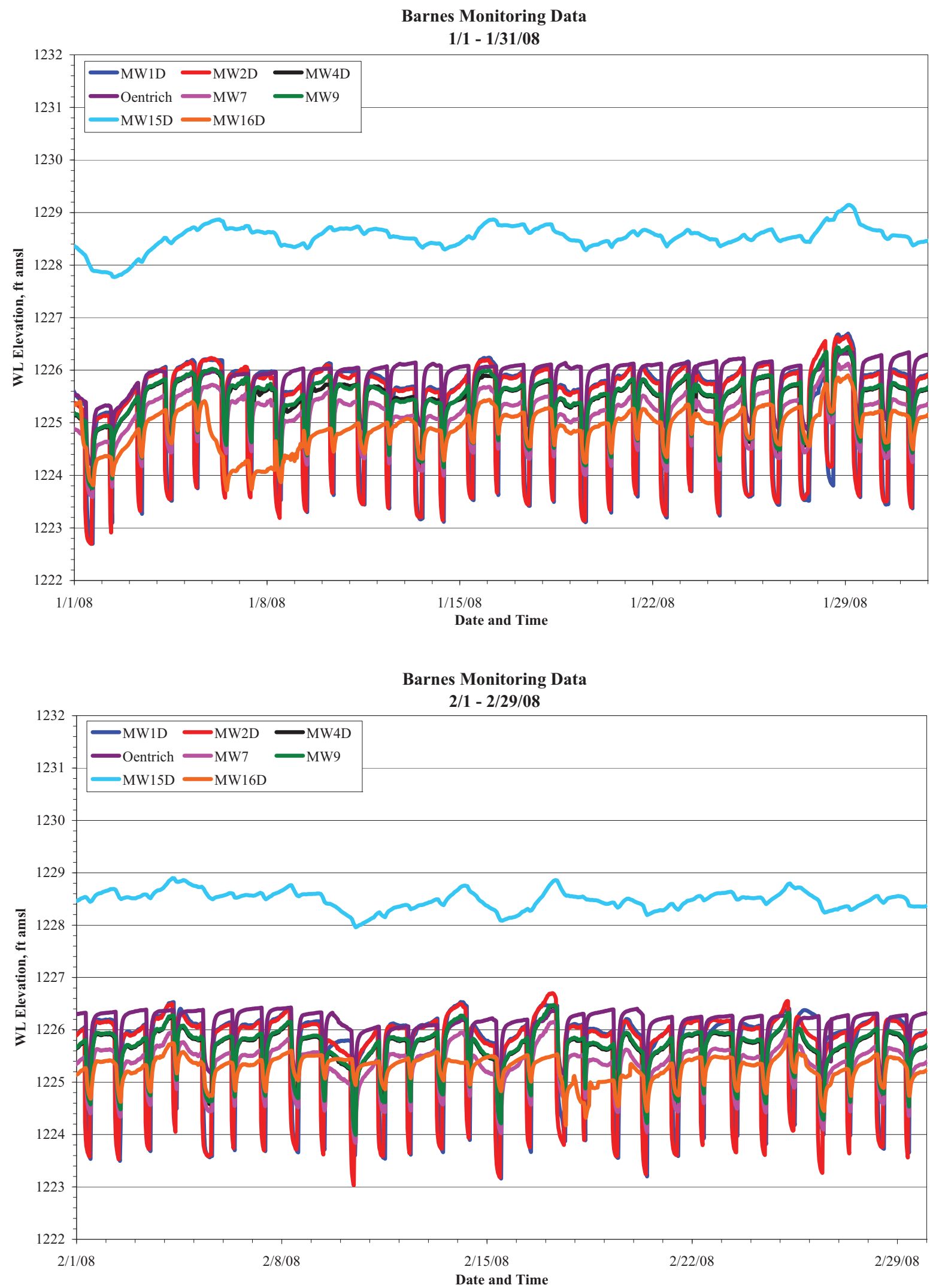

FIGURE 3.2 Hydrographs summarizing monthly results of long-term water level monitoring in wells at Barnes, January to November 2008. 

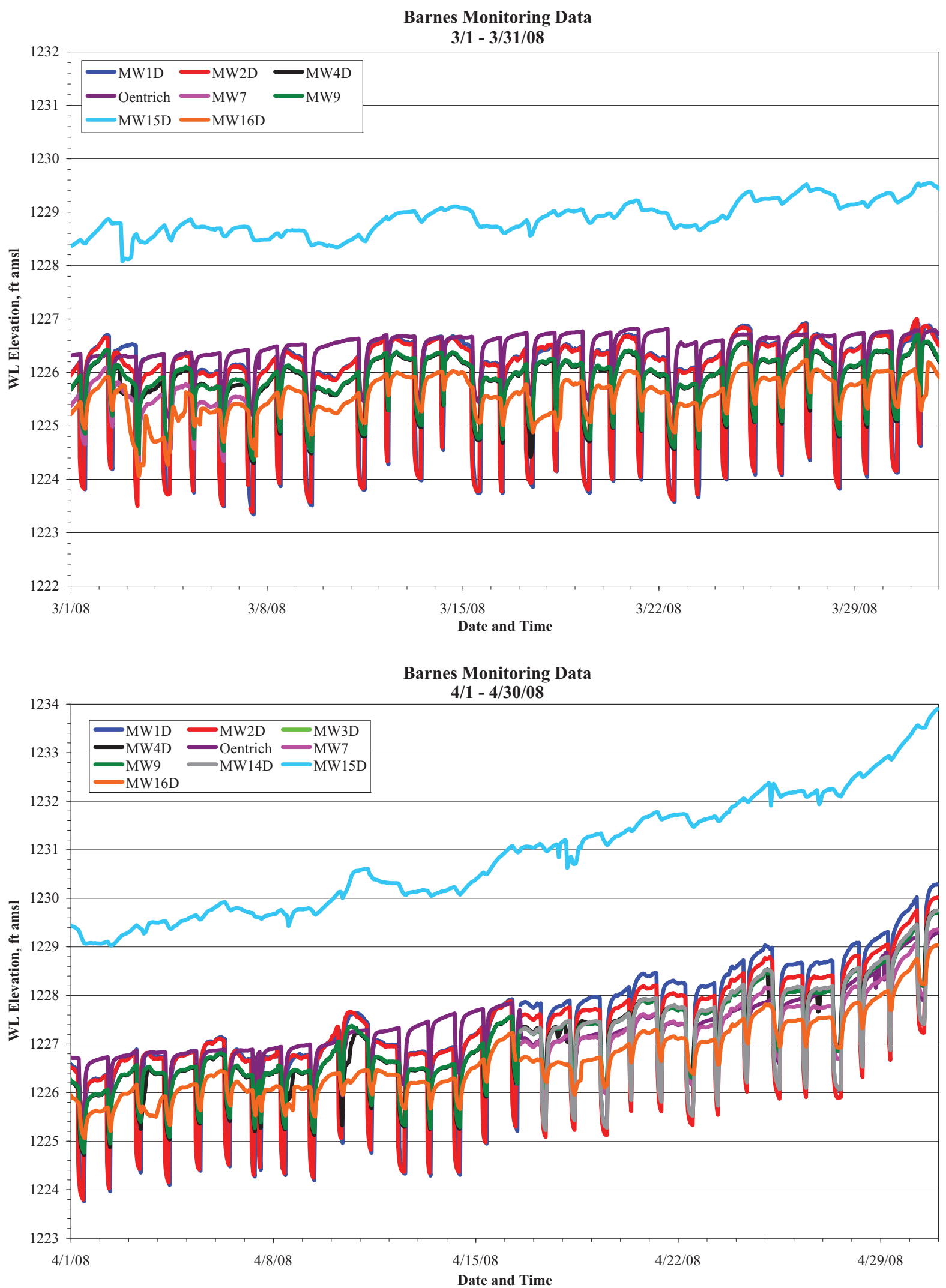

FIGURE 3.2 (cont.) 

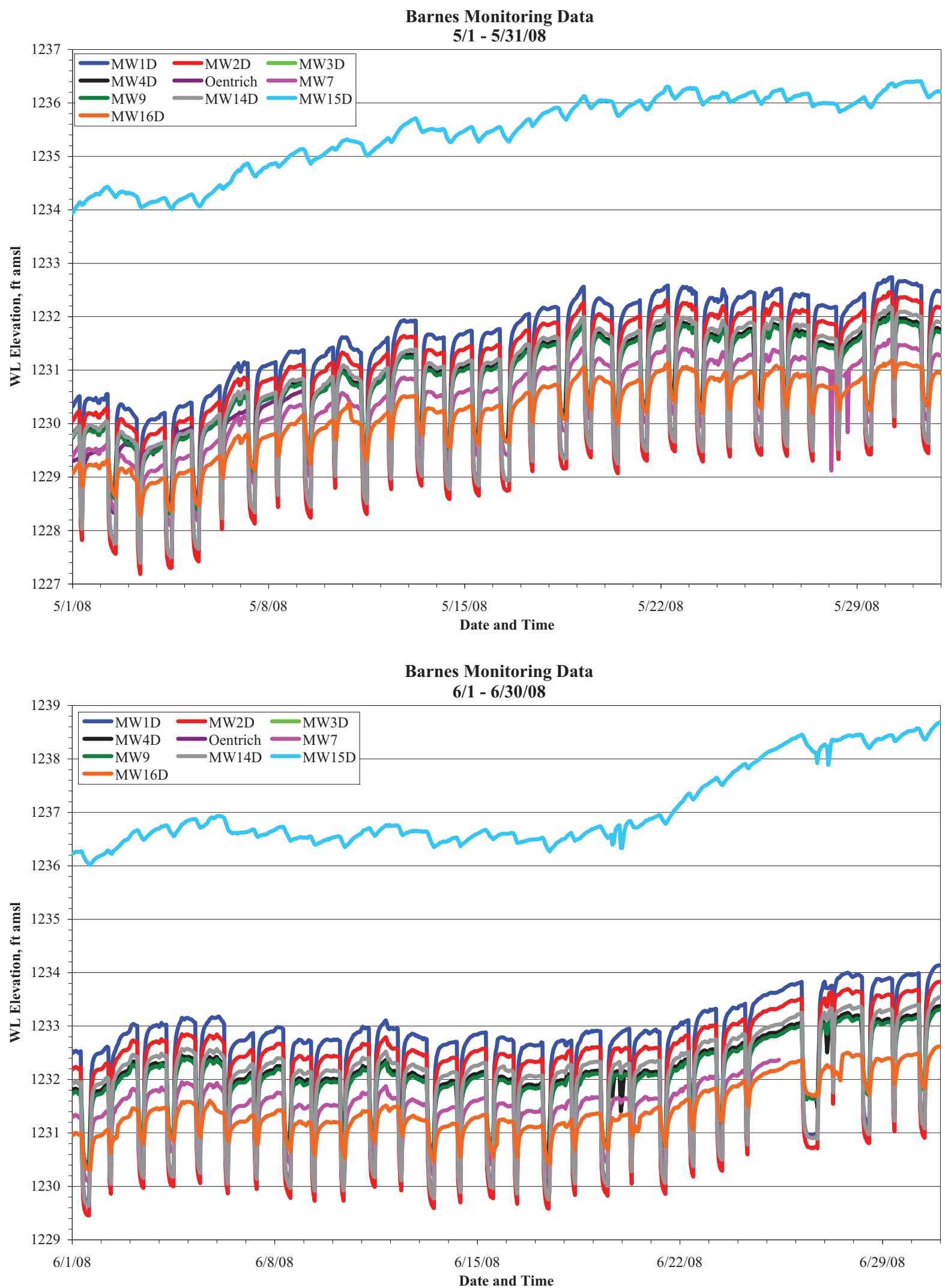

FIGURE 3.2 (cont.) 

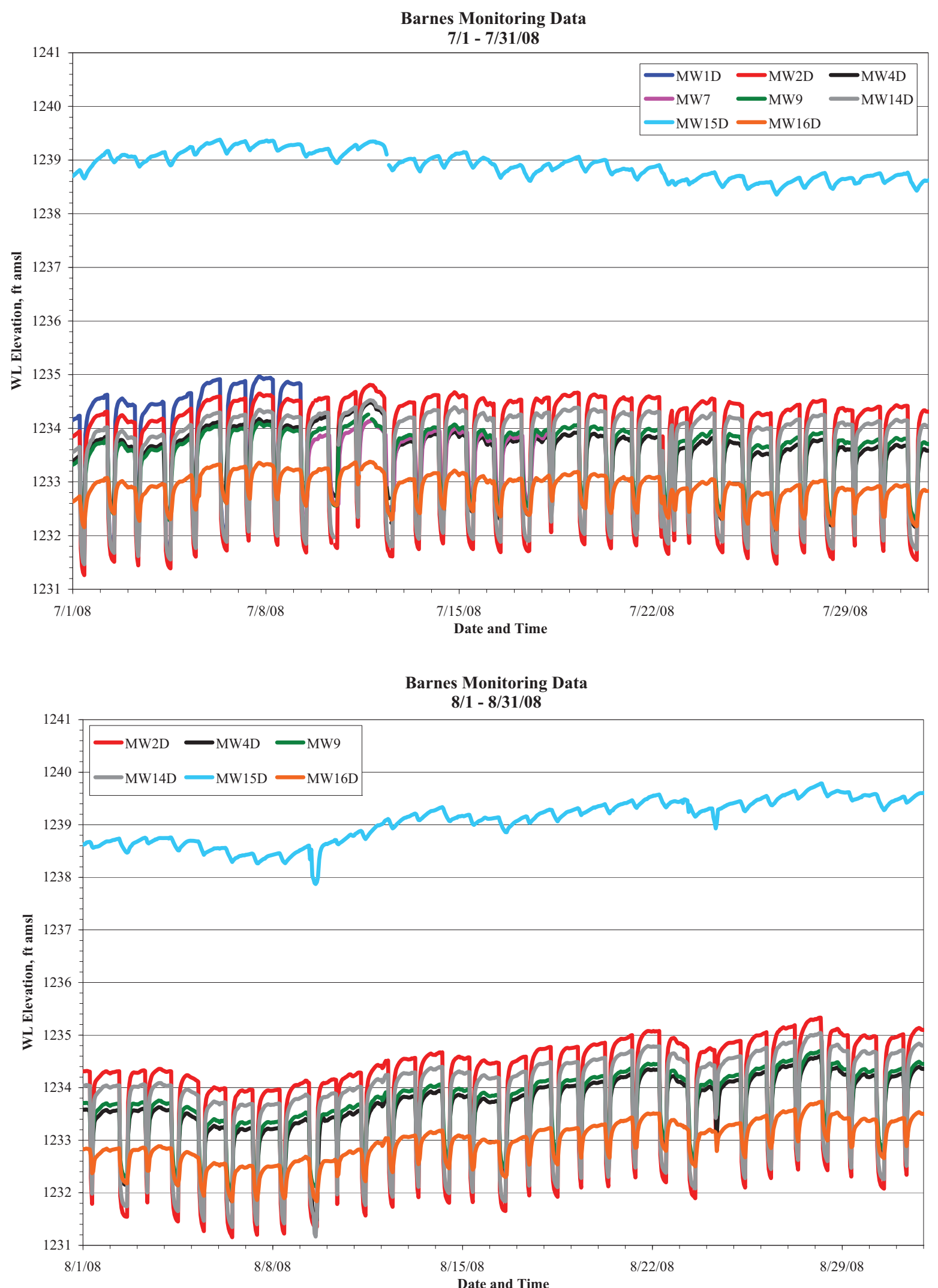

FIGURE 3.2 (cont.) 

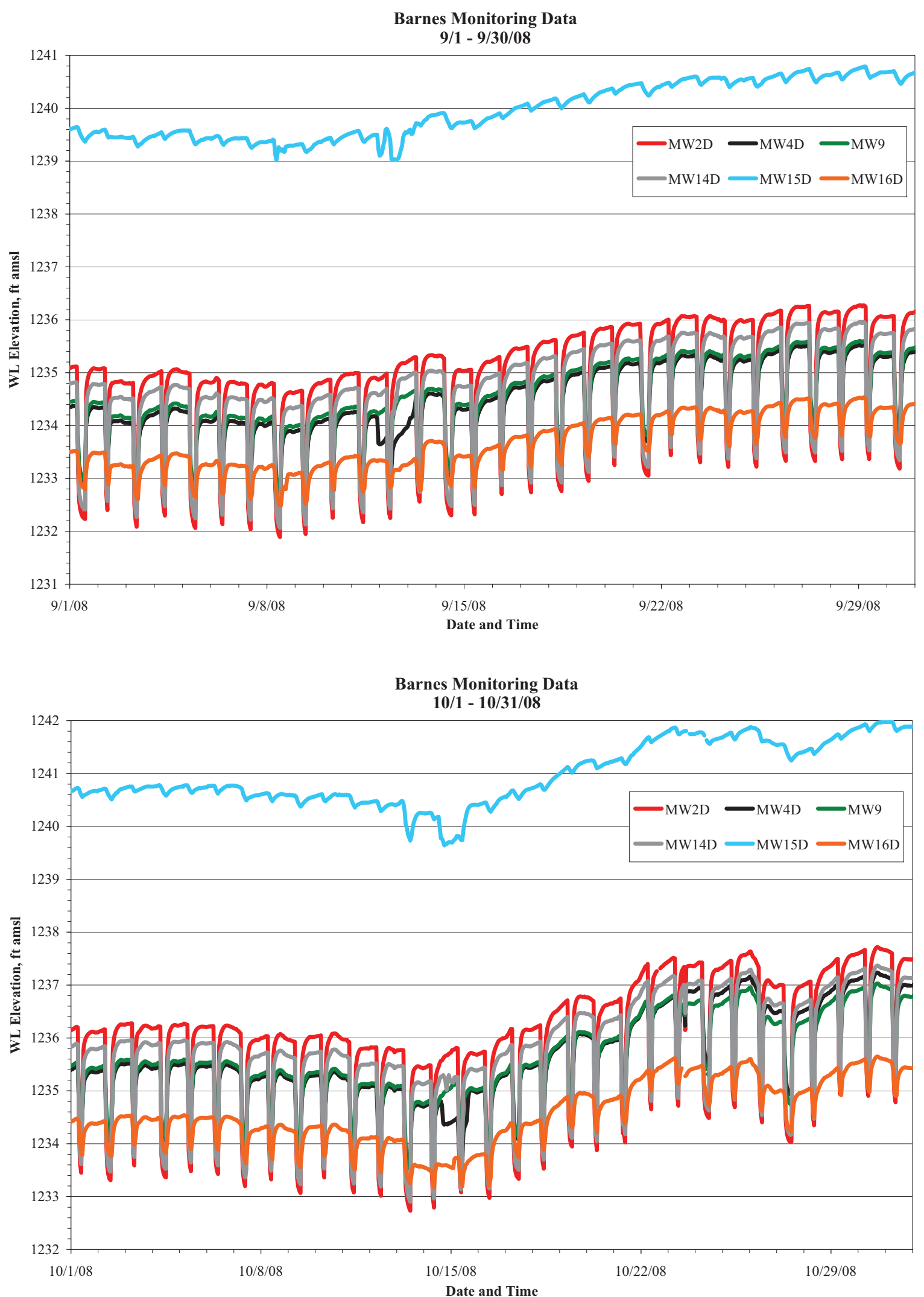

FIGURE 3.2 (cont.) 
October 2008 Monitoring Results for Barnes, Kansas

$3-21$

Version 00, 02/03/09

Barnes Monitoring Data 11/1 - 11/31/08

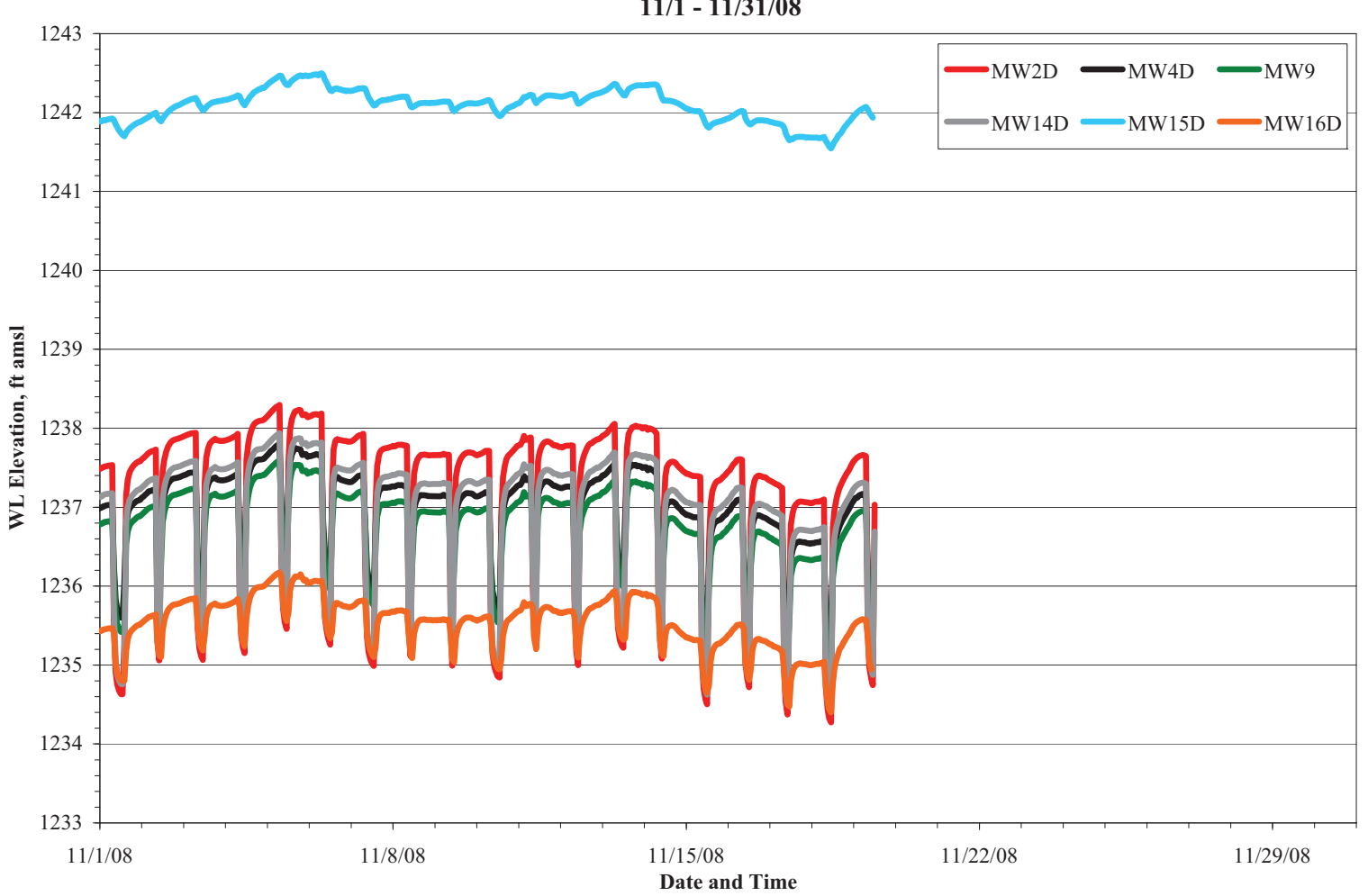

FIGURE 3.2 (cont.) 


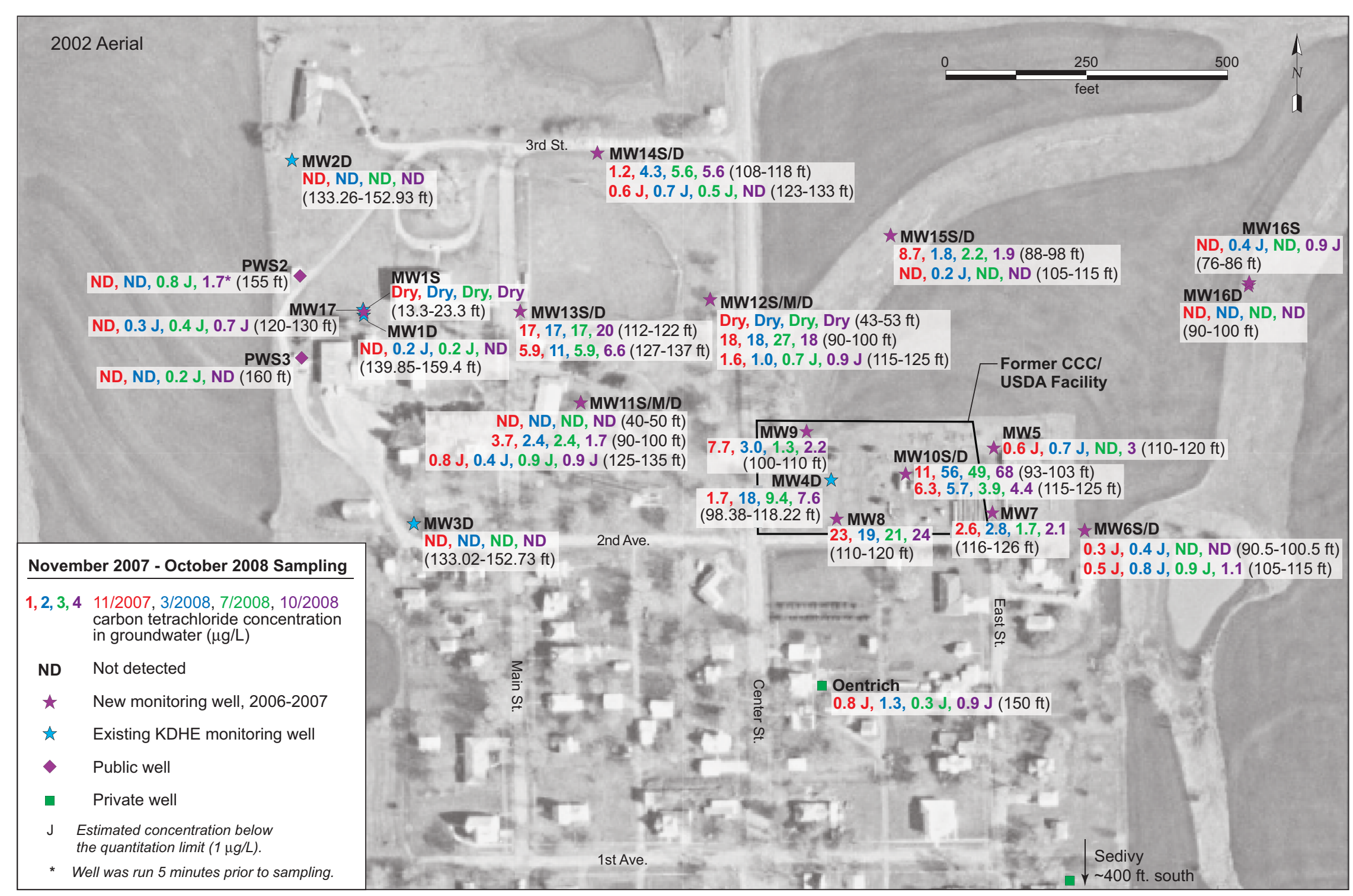

FIGURE 3.3 Analytical results for carbon tetrachloride in groundwater samples collected at Barnes in November 2007 to October 2008. Source of photograph: NAPP (2002). 


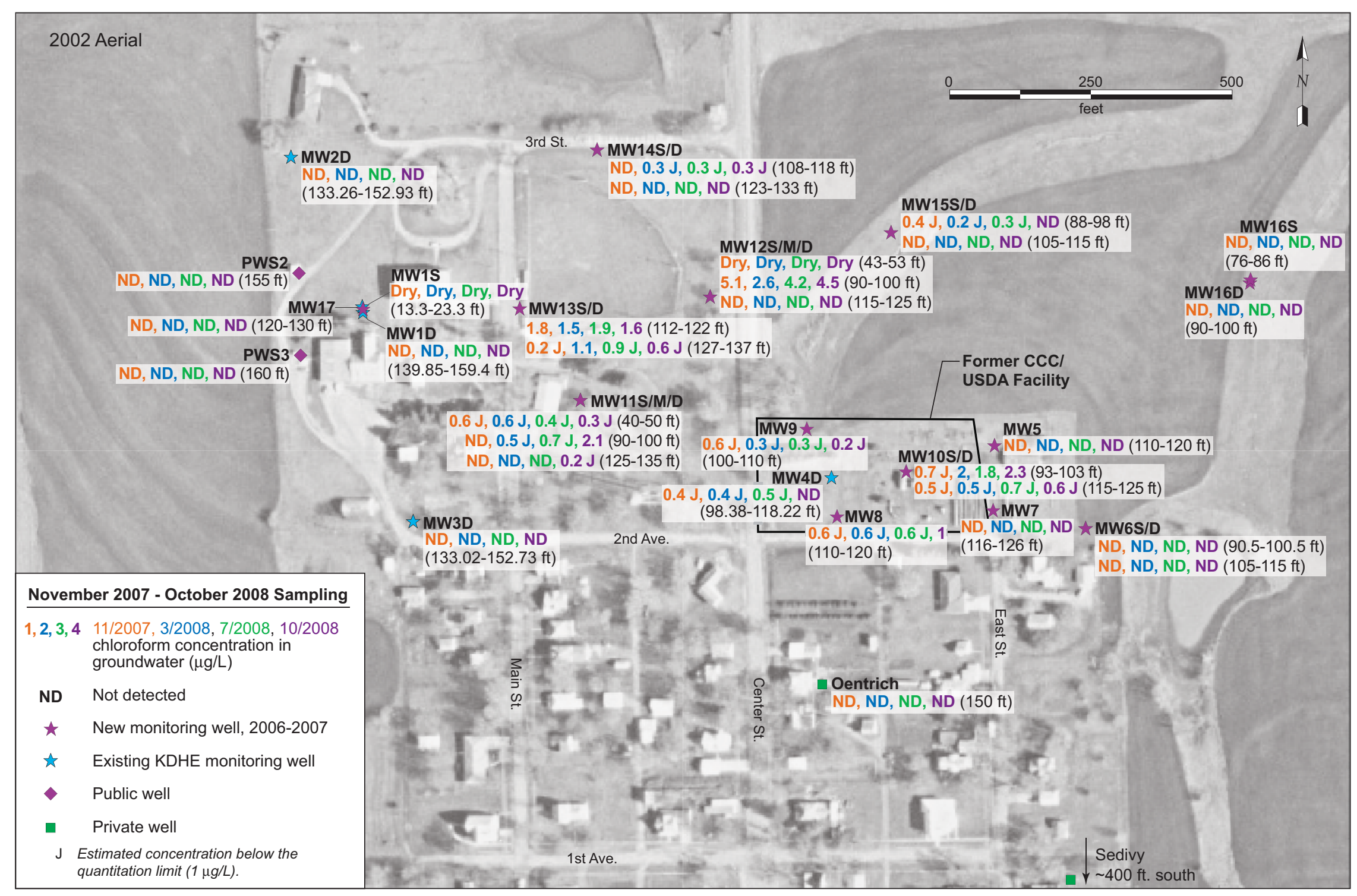

FIGURE 3.4 Analytical results for chloroform in groundwater samples collected at Barnes in November 2007 to October 2008. Source of photograph: NAPP (2002). 


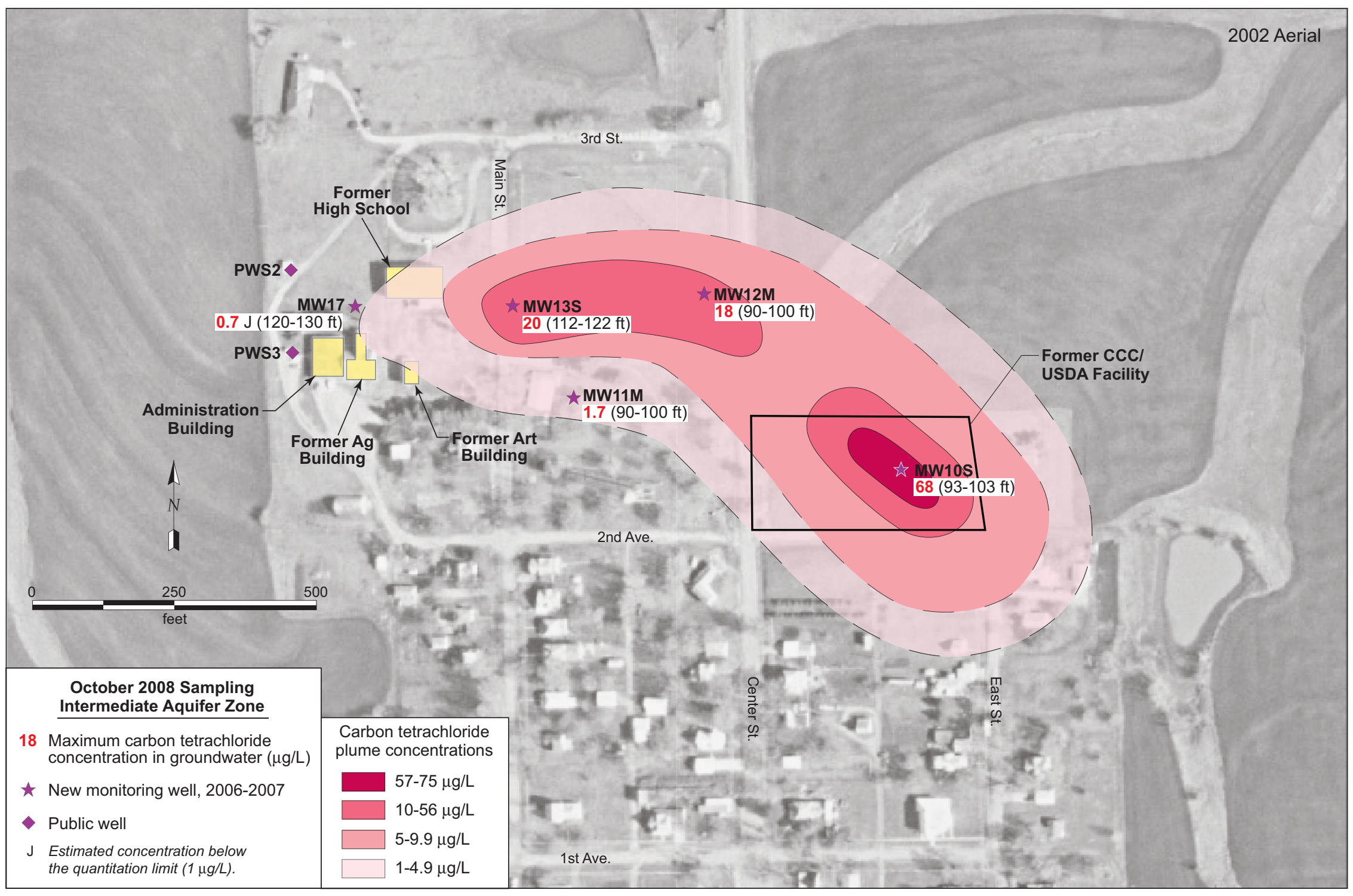

FIGURE 3.5 Interpreted carbon tetrachloride plume in October 2008 in wells screened in the intermediate aquifer zone (groundwater levels approximately 1,256-1,259 ft AMSL). Source of photograph: NAPP (2002). 


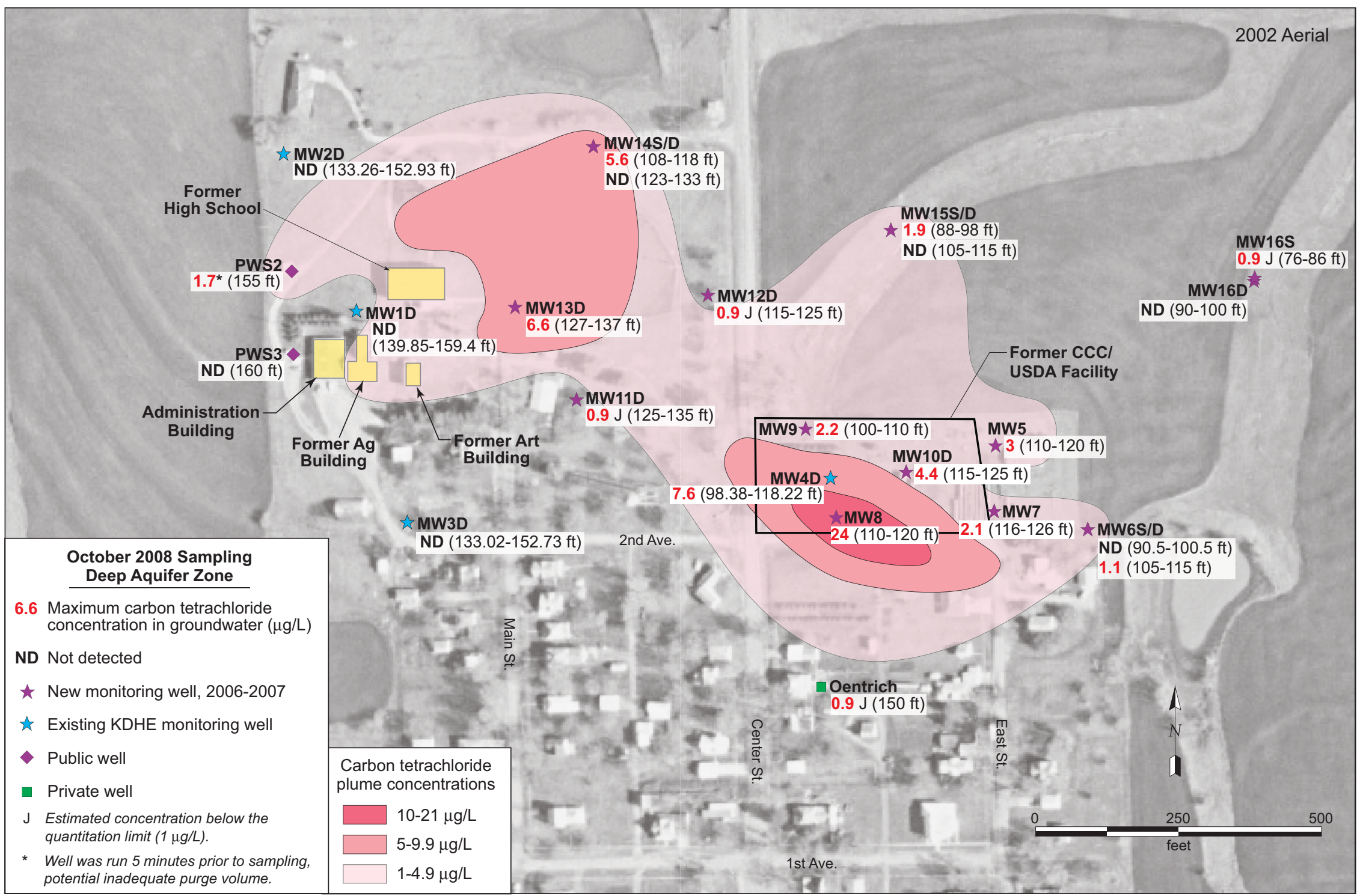

FIGURE 3.6 Interpreted carbon tetrachloride plume in October 2008 in wells screened in the deep aquifer zone (groundwater levels approximately 1,235-1,242 ft AMSL). Source of photograph: NAPP (2002). 


\section{Conclusions and Ongoing Tasks}

\subsection{Conclusions}

The findings of the October 2008 monitoring event at Barnes support the following conclusions:

- Measurements of groundwater levels obtained manually and through the use of automatic recorders have consistently indicated that the flow direction is strongly influenced by pumping of the public water supply wells. The results have demonstrated

- An apparent groundwater flow direction to the northeast when the public wells are not pumping and

- A northwesterly groundwater flow trend when the public wells are pumping.

- Evaluation of manual water level measurements and carbon tetrachloride concentrations continued to suggest that three vertically distinguishable aquifer zones are present at Barnes: a shallow zone with a potentiometric surface at approximately $1,314 \mathrm{ft}$ AMSL, an intermediate zone at approximately 1,256-1,259 ft AMSL, and a deep zone at approximately 1,235-1,242 ft AMSL (based on measurements taken in October 2008). As of November 2008, all 5 monitoring wells screened in the intermediate aquifer zone and 7 of 20 wells completed in the deep aquifer zone are equipped with automatic water level recorders Additional recorders will be added as warranted and as they become available from the manufacturer.

- The vertical distribution of the carbon tetrachloride in groundwater continues to indicate that the highest concentrations occur in the intermediate aquifer zone. Lower concentrations have been detected in the deep aquifer zone, and no carbon tetrachloride has been detected in the shallow zone. 
- Use of the low-flow sampling method during the three monitoring events from March to October 2008 indicated that the maximum level of carbon tetrachloride identified in sitewide monitoring has consistently been detected at intermediate-zone well MW10S, located in the eastern portion of the former CCC/USDA facility.

- Overall, the lateral distribution of carbon tetrachloride in groundwater in October 2008 is similar to the distribution during previous sampling events. The most significant changes are an increase in the concentration of carbon tetrachloride in public well PWS2 (from $<1 \mu \mathrm{g} / \mathrm{L}$ in July to $1.7 \mu \mathrm{g} / \mathrm{L}$ in October) and a decrease at PWS3 (from $<1 \mu \mathrm{g} / \mathrm{L}$ in July to no detection in October).

\subsection{Ongoing Tasks}

Ongoing tasks related to Barnes are as follows:

- Quarterly monitoring will continue, as recommended in the targeted investigation report (Argonne 2008a).

- In cooperation with the city, daily pumping rates are being recorded over a short time period to provide information for further evaluation of the aquifer characteristics. When this additional information is available, an attempt will be made to model the effects of the pumping (including rates and frequency) on the distribution of carbon tetrachloride in the subsurface. This effort is expected to include estimation of the capture zone for the pumping public water supply wells. The exact value of the effort will be predicated on the accuracy and level of detail of data provided by the city of Barnes for use in the modeling.

- The conceptual contingent interim measure work plan requested by the KDHE (2008) for protection of the public water supply wells is currently being prepared for submission. Cost estimates have been obtained for treatment at the wellhead through use of carbon filtration or air stripping. The CCC/USDA 
and Argonne have also contacted Washington County Rural Water District \#2 to determine the feasibility of connecting the city of Barnes to that water supply. 


\section{References}

Argonne, 2002, Final Master Work Plan: Environmental Investigations at Former CCC/USDA Facilities in Kansas, 2002 Revision, ANL/ER/TR-02/004, prepared for the Commodity Credit Corporation, U.S. Department of Agriculture, Washington, D.C., by Argonne National Laboratory, Argonne, Illinois, December.

Argonne, 2008a, Final Report: Results of the 2006-2007 Investigation of Potential Contamination at the Former CCC/USDA facility in Barnes, Kansas, ANL/EVS/AGEM/TR-0712, prepared for the Commodity Credit Corporation, U.S. Department of Agriculture, Washington, D.C., by Argonne National Laboratory, Argonne, Illinois, August.

Argonne, 2008b, November 2007 Monitoring Results for Barnes, Kansas, ANL/EVS/AGEM/TR-08-04, prepared for the Commodity Credit Corporation, U.S. Department of Agriculture, Washington, D.C., by Argonne National Laboratory, Argonne, Illinois, February.

Argonne, 2008c, March 2008 Monitoring Results for Barnes, Kansas, ANL/EVS/AGEM/TR-0811, prepared for the Commodity Credit Corporation, U.S. Department of Agriculture, Washington, D.C., by Argonne National Laboratory, Argonne, Illinois, August.

Argonne, 2008d, July 2008 Monitoring Results for Barnes, Kansas, ANL/EVS/AGEM/TR-0817, prepared for the Commodity Credit Corporation, U.S. Department of Agriculture, Washington, D.C., by Argonne National Laboratory, Argonne, Illinois, September.

EPA, 1995, Method 524.2: Measurement of Purgeable Organic Compounds in Water by Capillary Column Gas Chromatography/Mass Spectrometry, Revision 4.1, edited by J.W. Munch, National Exposure Research Laboratory, Office of Research and Development, U.S. Environmental Protection Agency, Cincinnati, Ohio.

KDHE, 2008, letter from C. Carey (Bureau of Environmental Remediation, Kansas Department of Health and Environment, Topeka, Kansas) to C. Roe (Commodity Credit Corporation, U.S. Department of Agriculture, Washington, D.C.), regarding the former CCC/USDA facility at Barnes, March 13. 
NAPP, 2002, aerial image NAPP 12897 222, National Aerial Photography Program, U.S. Geological Survey (available from Kansas Geospatial Community Commons [http://www.kansasgis.org/]), April 9.

Puls, R.W., and M.J. Barcelona, 1996, "Low-Flow (Minimal Drawdown) Ground-Water Sampling Procedures,” EPA/540/S-95/504, in Ground Water Issue, Superfund Technology Support Center for Ground Water, National Risk Management Research Laboratory, U.S. Environmental Protection Agency, Ada, Oklahoma, April (www.epa.gov/tio/tsp/download/ lwflw2a.pdf).

Yeskis, D., and B. Zavala, 2002, Ground-Water Sampling Guidelines for Superfund and RCRA Project Managers: Ground Water Forum Issue Paper, EPA 542-S-02-001, Technology Innovative Office, Office of Solid Waste and Emergency Response, U.S. Environmental Protection Agency, Washington, D.C., May (http://www.epa.gov/tio/tsp/download/ gw_sampling_guide.pdf). 


\section{Appendix A:}

Sampling Activities and Field Measurements at Barnes in October 2008 
TABLE A.1 Sequence of sampling activities at Barnes in October 2008. ${ }^{a}$

\begin{tabular}{|c|c|c|c|c|c|c|c|c|}
\hline Date & Time & Sample & Type $^{b}$ & Location & $\begin{array}{l}\text { DepthC } \\
\text { (ft BGL) }\end{array}$ & $\begin{array}{l}\text { Chain of } \\
\text { Custody }\end{array}$ & $\begin{array}{l}\text { Shipment } \\
\text { Date }\end{array}$ & Sample Description \\
\hline 10/22/08 & $12: 24$ & BAMW17-W-27746 & MW & MW17 & $120-130$ & 6151 & $10 / 22 / 08$ & $\begin{array}{l}\text { Depth to water }=95.15 \mathrm{ft} \text {. Depth of } 2 \text {-in. } \\
\text { well = } 130 \mathrm{ft} \text {. Sample collected by using } \\
\text { low-flow bladder pump after purging of } \\
6 \mathrm{~L} \text {. Pump intake positioned at } 125 \mathrm{ft} \text {. } \\
\text { Aliquots collected for verification analysis } \\
\text { by Test America. }\end{array}$ \\
\hline 10/22/08 & $12: 30$ & BAQCIR-W-27753 & RI & QC & - & 6151 & $10 / 22 / 08$ & $\begin{array}{l}\text { Rinsate of decontaminated sampling line } \\
\text { after collection of sample BAMW17-W- } \\
27746 \text {. }\end{array}$ \\
\hline 10/22/08 & 13:00 & BAMW1S-dry-Oct08 & MW & MW1S & 13.3-23.3 & - & - & Monitoring well MW1S was dry. \\
\hline 10/22/08 & 13:12 & BAMW3D-W-27722 & MW & MW3D & $133.02-152.73$ & 6151 & 10/22/08 & $\begin{array}{l}\text { Depth to water }=108.5 \mathrm{ft} \text {. Depth of } 2 \text {-in. } \\
\text { well }=152.73 \mathrm{ft} \text {. Sample collected by using } \\
\text { low-flow bladder pump after purging of } \\
10 \mathrm{~L} \text {. Pump intake positioned at } 142.88 \mathrm{ft} \text {. } \\
\text { Aliquots collected for verification analysis } \\
\text { by TestAmerica. }\end{array}$ \\
\hline 10/22/08 & $13: 40$ & - & - & MW1S & 13.3-23.3 & - & - & Monitoring Well MW1S was dry. \\
\hline 10/22/08 & $13: 48$ & BAMW1D-W-27720 & MW & MW1D & $139.85-159.4$ & 6151 & $10 / 22 / 08$ & $\begin{array}{l}\text { Depth to water = } 113.77 \mathrm{ft} \text {. Depth of } 2 \text {-in. } \\
\text { well = } 159.4 \mathrm{ft} \text {. Sample collected by using } \\
\text { low-flow bladder pump after purging of } \\
4 \mathrm{~L} \text {. Pump intake positioned at } 149.63 \mathrm{ft} \text {. }\end{array}$ \\
\hline 10/22/08 & $13: 49$ & BAMW1DDUP-W-27750 & MW & MW1D & $139.85-159.4$ & 6151 & $10 / 22 / 08$ & Replicate of sample BAMW1D-W-27720. \\
\hline $10 / 22 / 08$ & $14: 12$ & BAMW13S-W-27738 & MW & MW13S & $112-122$ & 6151 & $10 / 22 / 08$ & $\begin{array}{l}\text { Depth to water }=86 \mathrm{ft} \text {. Depth of } 2 \text {-in. well = } \\
122 \mathrm{ft} \text {. Sample collected by using low-flow } \\
\text { bladder pump after purging of } 4.5 \mathrm{~L} \text {. Pump } \\
\text { intake positioned at } 117 \mathrm{ft} \text {. }\end{array}$ \\
\hline 10/22/08 & $14: 13$ & $\begin{array}{l}\text { BAMW13SDUP-W- } \\
27751\end{array}$ & MW & MW13S & $112-122$ & 6151 & 10/22/08 & Replicate of sample BAMW13S-W-27738. \\
\hline 10/22/08 & $15: 30$ & BAMW13D-W-27739 & MW & MW13D & $127-137$ & 6151 & $10 / 22 / 08$ & $\begin{array}{l}\text { Depth to water = } 105.3 \mathrm{ft} \text {. Depth of } 2 \text {-in. } \\
\text { well = } 137 \mathrm{ft} \text {. Sample collected by using } \\
\text { low-flow bladder pump after purging of } \\
5 \mathrm{~L} \text {. Pump intake positioned at } 132 \mathrm{ft} \text {. }\end{array}$ \\
\hline 10/22/08 & $15: 40$ & BAQCIR-W-27752 & RI & QC & - & 6151 & $10 / 22 / 08$ & $\begin{array}{l}\text { Rinsate of decontaminated sampling line } \\
\text { after collection of sample BAMW13D-W- } \\
27739 \text {. }\end{array}$ \\
\hline 10/22/08 & $16: 00$ & BAQCTB-W-27754 & TB & QC & - & 6151 & $10 / 22 / 08$ & $\begin{array}{l}\text { Trip blank with water samples to the AGEM } \\
\text { Laboratory for organic analysis listed on } \\
\text { chain-of-custody form (COC) } 6151 \text {. }\end{array}$ \\
\hline
\end{tabular}


TABLE A.1 (Cont.)

\begin{tabular}{|c|c|c|c|c|c|c|c|c|}
\hline Date & Time & Sample & Type $^{b}$ & Location & $\begin{array}{l}\text { Depthc } \\
\text { (ft BGL) }\end{array}$ & $\begin{array}{l}\text { Chain of } \\
\text { Custody }\end{array}$ & $\begin{array}{l}\text { Shipment } \\
\text { Date }\end{array}$ & Sample Description \\
\hline 10/22/08 & $16: 22$ & BAMW2D-W-27721 & MW & MW2D & $133.26-152.93$ & 6151 & $10 / 22 / 08$ & $\begin{array}{l}\text { Depth to water = } 113.55 \mathrm{ft} \text {. Depth of } 2 \text {-in. } \\
\text { well = } 152.93 \mathrm{ft} \text {. Sample collected by using } \\
\text { low-flow bladder pump after purging of } \\
7 \mathrm{~L} \text {. Pump intake positioned at } 143.10 \mathrm{ft} \text {. }\end{array}$ \\
\hline 10/22/08 & $16: 46$ & BAMW14S-W-27740 & MW & MW14S & $108-118$ & 6153 & 10/23/08 & $\begin{array}{l}\text { Depth to water }=96.2 \mathrm{ft} \text {. Depth of } 2 \text {-in. } \\
\text { well = } 118 \mathrm{ft} \text {. Sample collected by using } \\
\text { low-flow bladder pump after purging of } \\
4.5 \mathrm{~L} \text {. Pump intake positioned at } 113 \mathrm{ft} \text {. }\end{array}$ \\
\hline 10/22/08 & $17: 00$ & BAMW12S-dry-Oct08 & MW & MW12S & $43-53$ & - & - & Monitoring well MW12S was dry. \\
\hline 10/22/08 & $17: 45$ & BAMW12M-W-27736 & MW & MW12M & $90-100$ & 6153 & 10/23/08 & $\begin{array}{l}\text { Depth to water }=69.72 \mathrm{ft} \text {. Depth of } 2 \text {-in. } \\
\text { well }=99 \mathrm{ft} \text {. Sample collected by using } \\
\text { low-flow bladder pump after purging of } \\
4 \mathrm{~L} \text {. Pump intake positioned at } 95 \mathrm{ft} \text {. }\end{array}$ \\
\hline $10 / 22 / 08$ & 18:02 & BAMW14D-W-27741 & MW & MW14D & $123-133$ & 6153 & 10/23/08 & $\begin{array}{l}\text { Depth to water = } 96.1 \mathrm{ft} \text {. Depth of } 2 \text {-in. } \\
\text { well = } 133 \mathrm{ft} \text {. Sample collected by using } \\
\text { low-flow bladder pump after purging of } \\
7.2 \mathrm{~L} \text {. Pump intake positioned at } 128 \mathrm{ft} \text {. }\end{array}$ \\
\hline 10/22/08 & $18: 48$ & BAMW12D-W-27737 & MW & MW12D & $115-125$ & 6153 & $10 / 23 / 08$ & $\begin{array}{l}\text { Depth to water }=91.12 \mathrm{ft} \text {. Depth of } 2 \text {-in. } \\
\text { well = } 125 \mathrm{ft} \text {. Sample collected by using } \\
\text { low-flow bladder pump after purging of } \\
6 \mathrm{~L} \text {. Pump intake positioned at } 120 \mathrm{ft} \text {. }\end{array}$ \\
\hline 10/23/08 & 10:04 & BAMW11S-W-27732 & MW & MW11S & $40-50$ & 6154 & 10/23/08 & $\begin{array}{l}\text { Depth to water }=22.5 \mathrm{ft} \text {. Depth of } 1 \mathrm{in} \text {. } \\
\text { well = } 50 \mathrm{ft} \text {. Sample collected by using } \\
\text { low-flow bladder pump after purging of } \\
2.5 \mathrm{~L} \text {. Pump intake positioned at } 45 \mathrm{ft} \text {. } \\
\text { Aliquots for collected verification analysis } \\
\text { by TestAmerica. }\end{array}$ \\
\hline 10/23/08 & $10: 18$ & BAMW7-W-27727 & MW & MW7 & $116-126$ & 6154 & 10/23/08 & $\begin{array}{l}\text { Depth to water = } 94.9 \mathrm{ft} \text {. Depth of } 2 \text {-in. } \\
\text { well = } 126 \mathrm{ft} \text {. Sample collected by using } \\
\text { low-flow bladder pump after purging of } \\
10 \mathrm{~L} \text {. Pump intake positioned at } 121 \mathrm{ft} \text {. } \\
\text { Very sandy water, tan in color. Aliquots } \\
\text { collected for verification analysis by } \\
\text { TestAmerica. }\end{array}$ \\
\hline
\end{tabular}


TABLE A.1 (Cont.)

\begin{tabular}{|c|c|c|c|c|c|c|c|c|}
\hline Date & Time & Sample & Type $^{b}$ & Location & $\begin{array}{l}\text { Depthc }^{\mathrm{C}} \\
\text { (ft BGL) }\end{array}$ & $\begin{array}{l}\text { Chain of } \\
\text { Custody }\end{array}$ & $\begin{array}{l}\text { Shipment } \\
\text { Date }\end{array}$ & Sample Description \\
\hline $10 / 23 / 08$ & $11: 03$ & BAMW11D-W-27734 & MW & MW11D & $125-135$ & 6154 & $10 / 23 / 08$ & $\begin{array}{l}\text { Depth to water }=101.03 \mathrm{ft} \text {. Depth of } 2 \text {-in. } \\
\text { well = } 135 \mathrm{ft} \text {. Sample collected by using } \\
\text { low-flow bladder pump after purging of } \\
6 \mathrm{~L} . \text { Pump intake positioned at } 130 \mathrm{ft} \text {. }\end{array}$ \\
\hline $10 / 23 / 08$ & $11: 28$ & BAMW5-W-27724 & MW & MW5 & $110-120$ & 6154 & $10 / 23 / 08$ & $\begin{array}{l}\text { Depth to water }=91.4 \mathrm{ft} \text {. Depth of } 2 \text {-in. } \\
\text { well = } 120 \mathrm{ft} \text {. Sample collected by using } \\
\text { low-flow bladder pump after purging of } \\
5.8 \mathrm{~L} \text {. Pump intake positioned at } 115 \mathrm{ft} \text {. }\end{array}$ \\
\hline $10 / 23 / 08$ & $12: 00$ & BAMW11M-W-27733 & MW & MW11M & $90-100$ & 6154 & 10/23/08 & $\begin{array}{l}\text { Depth to water }=77.8 \mathrm{ft} \text {. Depth of } 2 \text {-in. } \\
\text { well = } 100 \mathrm{ft} \text {. Sample collected by using } \\
\text { low-flow bladder pump after purging of } \\
4.5 \mathrm{~L} \text {. Pump intake positioned at } 95 \mathrm{ft} \text {. }\end{array}$ \\
\hline $10 / 23 / 08$ & $12: 40$ & BAMW10S-W-27730 & MW & MW10S & $93-103$ & 6154 & $10 / 23 / 08$ & $\begin{array}{l}\text { Depth to water }=72 \mathrm{ft} \text {. Depth of } 2 \text {-in. well = } \\
103 \mathrm{ft} \text {. Sample collected by using low-flow } \\
\text { bladder pump after purging of } 7 \mathrm{~L} \text {. Pump } \\
\text { intake positioned at } 98 \mathrm{ft} \text {. }\end{array}$ \\
\hline $10 / 23 / 08$ & $13: 16$ & BAMW16S-W-27744 & MW & MW16S & $76-86$ & 6154 & $10 / 23 / 08$ & $\begin{array}{l}\text { Depth to water }=64.8 \mathrm{ft} \text {. Depth of } 2 \text {-in. } \\
\text { well }=86 \mathrm{ft} . \text { Sample collected by using } \\
\text { low-flow bladder pump after purging of } \\
4.5 \mathrm{~L} \text {. Pump intake positioned at } 81 \mathrm{ft} \text {. }\end{array}$ \\
\hline $10 / 23 / 08$ & $13: 36$ & BAMW10D-W-27731 & MW & MW10D & $115-125$ & 6154 & $10 / 23 / 08$ & $\begin{array}{l}\text { Depth to water }=95 \mathrm{ft} \text {. Depth of } 2 \text {-in. well = } \\
125 \mathrm{ft} \text {. Sample collected by using low-flow } \\
\text { bladder pump after purging of } 5 \mathrm{~L} \text {. Pump } \\
\text { intake positioned at } 120 \mathrm{ft} \text {. }\end{array}$ \\
\hline $10 / 23 / 08$ & $14: 10$ & BAMW16D-W-27745 & MW & MW16D & $90-100$ & 6154 & 10/23/08 & $\begin{array}{l}\text { Depth to water = } 63.9 \mathrm{ft} \text {. Depth of } 2 \text {-in. } \\
\text { well = } 100 \mathrm{ft} \text {. Sample collected by using } \\
\text { low-flow bladder pump after purging of } \\
4.75 \mathrm{~L} \text {. Pump intake positioned at } 95 \mathrm{ft} \text {. }\end{array}$ \\
\hline $10 / 23 / 08$ & $14: 44$ & BAMW8-W-27728 & MW & MW8 & $110-120$ & 6154 & $10 / 23 / 08$ & $\begin{array}{l}\text { Depth to water = } 93.4 \mathrm{ft} \text {. Depth of } 2 \text {-in. } \\
\text { well = } 120 \mathrm{ft} \text {. Sample collected by using } \\
\text { low-flow bladder pump after purging of } \\
5 \mathrm{~L} \text {. Pump intake positioned at } 115 \mathrm{ft} \text {. }\end{array}$ \\
\hline $10 / 23 / 08$ & $15: 04$ & BAPW3-W-27749 & PW & PWS3 & 160 & 6154 & 10/23/08 & $\begin{array}{l}\text { Well was running for } 0.5 \mathrm{hr} \text {, then sampled at } \\
\text { tap in well shed. }\end{array}$ \\
\hline $10 / 23 / 08$ & $15: 10$ & BAPW2-W-27748 & PW & PWS2 & 155 & 6154 & $10 / 23 / 08$ & $\begin{array}{l}\text { Well was running for } 5 \text { min, then sampled at } \\
\text { tap in well shed. }\end{array}$ \\
\hline $10 / 23 / 08$ & $15: 20$ & BAOENTRICH-W-27747 & DW & Oentrich & 150 & 6154 & 10/23/08 & $\begin{array}{l}\text { Sampled from hydrant in back yard. Allowed } \\
\text { to run for } 5 \text { min and then sampled. }\end{array}$ \\
\hline
\end{tabular}


TABLE A.1 (Cont.)

\begin{tabular}{|c|c|c|c|c|c|c|c|c|}
\hline Date & Time & Sample & Type $^{b}$ & Location & $\begin{array}{l}\text { Depthc } \\
\text { (ft BGL) }\end{array}$ & $\begin{array}{l}\text { Chain of } \\
\text { Custody }\end{array}$ & $\begin{array}{l}\text { Shipment } \\
\text { Date }\end{array}$ & Sample Description \\
\hline 10/23/08 & $16: 03$ & BAMW4D-W-27723 & MW & MW4D & $98.38-118.22$ & 6154 & 10/23/08 & $\begin{array}{l}\text { Depth to water }=89.9 \mathrm{ft} \text {. Depth of } 2 \text {-in. } \\
\text { well }=118.22 \mathrm{ft} \text {. Sample collected by using } \\
\text { low-flow bladder pump after purging of } \\
6.5 \mathrm{~L} \text {. Pump intake positioned at } 108.30 \mathrm{ft} \text {. }\end{array}$ \\
\hline 10/23/08 & $16: 30$ & BAQCTB-W-27755 & TB & QC & - & 6153 & 10/23/08 & $\begin{array}{l}\text { Trip blank sent to the AGEM Laboratory for } \\
\text { organic analysis with water samples listed } \\
\text { on COCs } 6153 \text { and } 6154 \text {, as well as to } \\
\text { TestAmerica with verification samples } \\
\text { listed on COC } 6155 \text {. }\end{array}$ \\
\hline 10/23/08 & $16: 50$ & BAMW6D-W-27726 & MW & MW6D & $105-115$ & 6156 & $10 / 24 / 08$ & $\begin{array}{l}\text { Depth to water = } 87.15 \mathrm{ft} \text {. Depth of } 2 \text {-in. } \\
\text { well = } 115 \mathrm{ft} \text {. Sample collected by using } \\
\text { low-flow bladder pump after purging of } \\
4 \mathrm{~L} \text {. Pump intake positioned at } 110 \mathrm{ft} \text {. }\end{array}$ \\
\hline 10/23/08 & $16: 58$ & BAMW6S-W-27725 & MW & MW6S & $90.5-100.5$ & 6154 & $10 / 23 / 08$ & $\begin{array}{l}\text { Depth to water }=84.6 \mathrm{ft} \text {. Depth of } 2 \text {-in. } \\
\text { well = } 100.5 \mathrm{ft} \text {. Sample collected by using } \\
\text { low-flow bladder pump after purging of } \\
10 \mathrm{~L} \text {. Pump intake positioned at } 95.50 \mathrm{ft} \text {. }\end{array}$ \\
\hline 10/23/08 & $17: 42$ & BAMW15S-W-27742 & MW & MW15S & $88-98$ & 6156 & $10 / 24 / 08$ & $\begin{array}{l}\text { Depth to water }=73.2 \mathrm{ft} \text {. Depth of } 2 \text {-in. } \\
\text { well = } 98 \mathrm{ft} \text {. Sample collected by using } \\
\text { low-flow bladder pump after purging of } \\
12.5 \mathrm{~L} \text {. Pump intake positioned at } 93 \mathrm{ft} \text {. }\end{array}$ \\
\hline 10/24/08 & 9:00 & BAMW15D-W-27743 & MW & MW15D & $105-115$ & 6156 & $10 / 24 / 08$ & $\begin{array}{l}\text { Depth to water = } 67.6 \mathrm{ft} \text {. Depth of } 2 \text {-in. } \\
\text { well = } 115 \mathrm{ft} \text {. Sample collected by using } \\
\text { low-flow bladder pump after purging of } \\
5 \mathrm{~L} \text {. Pump intake positioned at } 110 \mathrm{ft} \text {. }\end{array}$ \\
\hline 10/24/08 & $10: 02$ & BAMW9-W-27729 & MW & MW9 & $100-110$ & 6156 & $10 / 24 / 08$ & $\begin{array}{l}\text { Depth to water = } 86.6 \mathrm{ft} \text {. Depth of } 2 \text {-in. } \\
\text { well = } 110 \mathrm{ft} \text {. Sample collected by using } \\
\text { low-flow bladder pump after purging of } \\
4.5 \mathrm{~L} \text {. Pump intake positioned at } 105 \mathrm{ft} \text {. }\end{array}$ \\
\hline 10/24/08 & $12: 00$ & BAQCTB-W-27756 & TB & QC & - & 6156 & $10 / 24 / 08$ & $\begin{array}{l}\text { Trip blank sent to the AGEM Laboratory for } \\
\text { organic analysis with water samples listed } \\
\text { on COC } 6156 \text {. }\end{array}$ \\
\hline
\end{tabular}


TABLE A.1 (Cont.)

All samples collected were water.

b Sample types: DW, domestic well; MW, monitoring well; PW, public water supply well; RI, rinsate; TB, trip blank.

c Depth is in feet below the top of the well casing 
TABLE A.2 Field measurements for groundwater samples collected at Barnes, July 2006 to October 2008.

\begin{tabular}{|c|c|c|c|c|c|c|c|}
\hline Well & $\begin{array}{l}\text { Screen Interval } \\
\quad(\mathrm{ft} B G L)\end{array}$ & $\begin{array}{l}\text { Sample } \\
\text { Date }\end{array}$ & $\begin{array}{c}\text { Temperature } \\
\left({ }^{\circ} \mathrm{C}\right)\end{array}$ & $\mathrm{pH}$ & $\begin{array}{c}\text { Conductivity } \\
(\mu \mathrm{S} / \mathrm{cm})\end{array}$ & $\begin{array}{c}\text { Dissolved } \\
\text { Oxygen } \\
(\mathrm{mg} / \mathrm{L})\end{array}$ & $\begin{array}{l}\text { Oxyge } \\
\text { Reduct } \\
\text { Potent } \\
(\mathrm{mV})\end{array}$ \\
\hline \multicolumn{8}{|c|}{ Previously existing KDHE monitoring wells } \\
\hline \multirow[t]{6}{*}{ MW1S } & $13.3-23.3$ & $7 / 19 / 06^{a}$ & - & - & - & - & - \\
\hline & & $4 / 4 / 07^{a}$ & - & _- & _- & _- & - \\
\hline & & $11 / 18 / 07^{a}$ & - & - & - & - & - \\
\hline & & $3 / 4 / 08^{a}$ & - & - & - & - & - \\
\hline & & $7 / 9 / 08^{a}$ & - & - & - & - & - \\
\hline & & $10 / 22 / 08^{a}$ & - & - & - & - & - \\
\hline \multirow[t]{6}{*}{ MW1D } & $139.85-159.4$ & 7/19/06 & 22.8 & 7.15 & 945 & - & - \\
\hline & & $4 / 4 / 07$ & 15.7 & 6.30 & 855 & - & - \\
\hline & & $11 / 18 / 07$ & 12.7 & 7.62 & 712 & - & - \\
\hline & & $3 / 4 / 08$ & 5.5 & 7.22 & 1167 & 11.6 & 244.2 \\
\hline & & $7 / 9 / 08$ & 18.1 & 7.05 & 992 & 16.2 & 97.5 \\
\hline & & $10 / 22 / 08$ & 12.6 & 7.07 & 937 & 8.95 & 107.9 \\
\hline \multirow[t]{6}{*}{ MW2D } & 133.26-152.93 & $7 / 19 / 06$ & 24.7 & 7.72 & 946 & - & - \\
\hline & & $4 / 4 / 07$ & 15.1 & 6.32 & 887 & - & - \\
\hline & & $11 / 18 / 07$ & 12.1 & 6.96 & 1448 & - & - \\
\hline & & $3 / 7 / 08$ & 6.5 & 7.22 & 1198 & 4.61 & 196.5 \\
\hline & & 7/10/08 & 18.4 & 6.91 & 1163 & 5.03 & 155.3 \\
\hline & & $10 / 22 / 08$ & 11.6 & 7.07 & 931 & 6.19 & 131.6 \\
\hline \multirow[t]{6}{*}{ MW3D } & $133.02-152.73$ & 7/19/06 & 23.0 & 7.06 & 976 & - & - \\
\hline & & $4 / 4 / 07$ & 15.6 & 6.37 & 989 & - & - \\
\hline & & $11 / 19 / 07$ & 10.5 & 7.16 & 1093 & - & - \\
\hline & & $3 / 7 / 08$ & 8.2 & 7.09 & 1195 & 5.34 & 254.8 \\
\hline & & 7/10/08 & 19.8 & 6.99 & 1177 & 13.8 & 109.9 \\
\hline & & $10 / 22 / 08$ & 13.5 & 7.01 & 1238 & 4.46 & 84.0 \\
\hline \multirow[t]{6}{*}{ MW4D } & $98.38-118.22$ & $7 / 20 / 06$ & 23.5 & 6.26 & 968 & - & - \\
\hline & & $4 / 6 / 07$ & 11.3 & 6.21 & 1018 & - & - \\
\hline & & $11 / 19 / 07$ & 15.7 & 6.98 & 1022 & - & - \\
\hline & & $3 / 9 / 08$ & 11.5 & 7.14 & 859 & 6.57 & 201.2 \\
\hline & & $7 / 12 / 08$ & 14.4 & 6.94 & 1001 & 6.77 & 148.7 \\
\hline & & $10 / 23 / 08$ & 13.0 & 7.02 & 973 & 5.56 & 93.5 \\
\hline
\end{tabular}

CCC/USDA wells installed during 2006-2007 investigation

\begin{tabular}{|c|c|c|c|c|c|c|c|}
\hline \multirow[t]{5}{*}{ MW5 } & $110-120$ & $4 / 6 / 07$ & 13.9 & 6.17 & 1705 & - & - \\
\hline & & $11 / 19 / 07$ & 15.2 & 6.74 & 3070 & - & - \\
\hline & & $3 / 8 / 08$ & 9.9 & 6.76 & 2770 & 0.66 & 123.2 \\
\hline & & 7/11/08 & 18.8 & 6.66 & 2930 & 1.32 & 36.6 \\
\hline & & 10/23/08 & 12.8 & 6.78 & 2384 & 0.67 & 20.4 \\
\hline \multirow[t]{5}{*}{ MW6S } & $90.5-100.5$ & $4 / 4 / 07^{a}$ & - & - & - & - & - \\
\hline & & $11 / 19 / 07$ & 12.0 & 7.60 & 723 & - & - \\
\hline & & $3 / 8 / 08$ & 4.7 & 7.77 & 673 & 6.72 & 272.2 \\
\hline & & 7/11/08 & 28.2 & 7.61 & 753 & 9.85 & 92.4 \\
\hline & & $10 / 23 / 08$ & 11.9 & $11.17^{b}$ & 582 & 5.12 & 91.0 \\
\hline
\end{tabular}


TABLE A.2 (Cont.)

\begin{tabular}{|c|c|c|c|c|c|c|c|}
\hline Well & $\begin{array}{l}\text { Screen Interval } \\
\quad \text { (ft BGL) }\end{array}$ & $\begin{array}{l}\text { Sample } \\
\text { Date }\end{array}$ & $\begin{array}{c}\text { Temperature } \\
\left({ }^{\circ} \mathrm{C}\right)\end{array}$ & $\mathrm{pH}$ & $\begin{array}{l}\text { Conductivity } \\
(\mu \mathrm{S} / \mathrm{cm})\end{array}$ & $\begin{array}{l}\text { Dissolved } \\
\text { Oxygen } \\
\text { (mg/L) }\end{array}$ & $\begin{array}{r}\text { Oxyge } \\
\text { Reduct } \\
\text { Potent } \\
(\mathrm{mV})\end{array}$ \\
\hline \multicolumn{8}{|c|}{ CCC/USDA wells installed during 2006-2007 investigation (cont.) } \\
\hline \multirow[t]{5}{*}{ MW6D } & $105-115$ & $4 / 5 / 07$ & 6.2 & 6.11 & 936 & - & - \\
\hline & & $11 / 19 / 07$ & 13.6 & 7.00 & 1103 & - & - \\
\hline & & $3 / 8 / 08$ & 9.1 & 7.15 & 908 & 5.56 & 241.0 \\
\hline & & $7 / 11 / 08$ & 19.8 & 7.05 & 999 & 12.8 & 99.9 \\
\hline & & $10 / 23 / 08$ & 12.0 & 7.18 & 957 & 6.27 & 127.5 \\
\hline \multirow[t]{5}{*}{ MW7 } & $116-126$ & $4 / 6 / 07$ & 14.1 & 6.30 & 1051 & - & - \\
\hline & & $11 / 19 / 07$ & 14.6 & 7.16 & 890 & - & - \\
\hline & & $3 / 9 / 08$ & 13.1 & 7.10 & 1068 & 4.24 & 186.4 \\
\hline & & $7 / 12 / 08$ & 14.4 & 6.95 & 1238 & 4.36 & 98.3 \\
\hline & & $10 / 23 / 08$ & 12.7 & 7.16 & 1191 & 8.61 & 121.8 \\
\hline \multirow[t]{5}{*}{ MW8 } & $110-120$ & $4 / 6 / 07$ & 12.1 & 6.23 & 974 & - & - \\
\hline & & $11 / 19 / 07$ & 14.6 & 7.03 & 909 & - & - \\
\hline & & $3 / 10 / 08$ & 13.1 & 7.09 & 961 & 6.71 & 182.1 \\
\hline & & $7 / 11 / 08$ & 18.6 & 6.38 & 1049 & 6.19 & 152.2 \\
\hline & & $10 / 23 / 08$ & 12.9 & 7.06 & 948 & 8.74 & 96.6 \\
\hline \multirow{5}{*}{ MW9 } & $100-110$ & $4 / 5 / 07$ & 12.9 & 6.20 & 976 & - & - \\
\hline & & 11/19/07 & 16.5 & 7.21 & 1066 & - & - \\
\hline & & $3 / 9 / 08$ & 11.2 & 7.07 & 928 & 5.80 & 239.0 \\
\hline & & $7 / 11 / 08$ & 17.7 & 6.58 & 1010 & 5.63 & 188.7 \\
\hline & & $10 / 24 / 08$ & 13.0 & 7.06 & 888 & 5.42 & 78.5 \\
\hline \multirow[t]{5}{*}{ MW10S } & $93-103$ & $4 / 6 / 07$ & 13.2 & 6.36 & 1004 & - & - \\
\hline & & 11/19/07 & 14.5 & 7.22 & 942 & - & - \\
\hline & & $3 / 10 / 08$ & 12.7 & 7.08 & 912 & 5.18 & 176.1 \\
\hline & & $7 / 11 / 08$ & 17.3 & 6.91 & 975 & 12.8 & 118.6 \\
\hline & & $10 / 23 / 08$ & 13.4 & 7.08 & 913 & 5.39 & 77.9 \\
\hline \multirow[t]{5}{*}{ MW10D } & $115-125$ & $4 / 6 / 07$ & 12.1 & 6.21 & 992 & - & - \\
\hline & & $11 / 19 / 07$ & 14.5 & 7.42 & 1175 & - & - \\
\hline & & $3 / 9 / 08$ & 13.7 & 7.01 & 1024 & 5.07 & 235.9 \\
\hline & & $7 / 11 / 08$ & 17.4 & 6.78 & 1090 & 12.6 & 117.1 \\
\hline & & $10 / 23 / 08$ & 13.5 & 7.01 & 1000 & 5.00 & 92.8 \\
\hline \multirow[t]{5}{*}{ MW11S } & $40-50$ & $4 / 4 / 07$ & 12.8 & 6.14 & 1027 & - & - \\
\hline & & $11 / 19 / 07$ & 11.2 & 7.15 & 1174 & - & - \\
\hline & & $3 / 5 / 08$ & 9.4 & 6.81 & 1122 & 2.26 & 240.8 \\
\hline & & $7 / 10 / 08$ & 19.5 & 6.47 & 1224 & 1.86 & 166.2 \\
\hline & & $10 / 23 / 08$ & 10.2 & 6.99 & 1085 & 5.80 & 146.4 \\
\hline \multirow[t]{5}{*}{ MW11M } & $90-100$ & $4 / 5 / 07$ & 7.5 & 7.60 & 1097 & - & - \\
\hline & & $11 / 19 / 07$ & 11.9 & 7.17 & 1144 & - & - \\
\hline & & 3/6/08 & 10.8 & 7.06 & 997 & 2.65 & 254.1 \\
\hline & & $7 / 10 / 08$ & 31.9 & 7.08 & 1124 & 3.88 & 148.9 \\
\hline & & $10 / 23 / 08$ & 12.4 & 7.06 & 962 & 3.19 & 116.1 \\
\hline
\end{tabular}


TABLE A.2 (Cont.)

\begin{tabular}{|c|c|c|c|c|c|c|c|}
\hline Well & $\begin{array}{l}\text { Screen Interval } \\
\quad(\mathrm{ft} B G L)\end{array}$ & $\begin{array}{l}\text { Sample } \\
\text { Date }\end{array}$ & $\begin{array}{c}\text { Temperature } \\
\left({ }^{\circ} \mathrm{C}\right)\end{array}$ & $\mathrm{pH}$ & $\begin{array}{l}\text { Conductivity } \\
(\mu \mathrm{S} / \mathrm{cm})\end{array}$ & $\begin{array}{l}\text { Dissolved } \\
\text { Oxygen } \\
\text { (mg/L) }\end{array}$ & $\begin{array}{l}\text { Oxygen } \\
\text { Reduction } \\
\text { Potential } \\
(\mathrm{mV})\end{array}$ \\
\hline \multicolumn{8}{|c|}{ CCC/USDA wells installed during 2006-2007 investigation (cont.) } \\
\hline \multirow[t]{5}{*}{ MW11D } & $125-135$ & $4 / 4 / 07$ & 13.8 & 6.18 & 990 & - & - \\
\hline & & $11 / 19 / 07$ & 13.1 & 7.22 & 987 & - & - \\
\hline & & 3/5/08 & 6.0 & 7.06 & 872 & 6.85 & 252.0 \\
\hline & & 7/10/08 & 17.5 & 6.25 & 957 & 7.14 & 176.6 \\
\hline & & 10/23/08 & 12.3 & 7.11 & 863 & 8.71 & 123.0 \\
\hline \multirow[t]{5}{*}{ MW12S } & $43-53$ & $4 / 5 / 07^{\mathrm{a}}$ & - & - & - & - & - \\
\hline & & $11 / 19 / 07^{a}$ & - & - & - & - & - \\
\hline & & $3 / 10 / 08^{a}$ & - & - & - & - & - \\
\hline & & $7 / 10 / 08^{a}$ & - & - & - & - & - \\
\hline & & $10 / 22 / 08^{a}$ & - & - & - & - & - \\
\hline \multirow{5}{*}{ MW12M } & $90-100$ & $4 / 5 / 07$ & 12.6 & 6.42 & 867 & - & - \\
\hline & & $11 / 19 / 07$ & 14.9 & 7.13 & 835 & - & - \\
\hline & & 3/10/08 & 12.6 & 7.13 & 665 & 1.81 & 211.5 \\
\hline & & 7/10/08 & 16.9 & 7.09 & 878 & 8.17 & 87.1 \\
\hline & & $10 / 22 / 08$ & 12.2 & 7.20 & 785 & 8.93 & 125.8 \\
\hline \multirow[t]{5}{*}{ MW12D } & $115-125$ & $4 / 5 / 07$ & 14.0 & 6.36 & 930 & - & - \\
\hline & & $11 / 18 / 07$ & 15.6 & 6.95 & 571 & - & - \\
\hline & & $3 / 9 / 08$ & 8.8 & 7.13 & 881 & 5.25 & 237.3 \\
\hline & & 7/11/08 & 19.9 & 6.01 & 987 & 4.72 & 196.5 \\
\hline & & 10/22/08 & 12.3 & 7.09 & 873 & 7.96 & 135.6 \\
\hline \multirow[t]{5}{*}{ MW13S } & $112-122$ & $4 / 5 / 07$ & 9.8 & 6.42 & 946 & - & - \\
\hline & & $11 / 19 / 07$ & 16.5 & 7.21 & 893 & - & - \\
\hline & & 3/10/08 & 12.2 & 7.13 & 810 & 6.21 & 199.3 \\
\hline & & 7/9/08 & 17.4 & 6.99 & 875 & 7.72 & 115.7 \\
\hline & & $10 / 22 / 08$ & 13.5 & 7.08 & 793 & 5.08 & 100.4 \\
\hline \multirow[t]{5}{*}{ MW13D } & $127-137$ & $4 / 5 / 07$ & 14.9 & 6.25 & 397 & - & - \\
\hline & & 11/19/07 & 17.0 & 7.00 & 763 & - & - \\
\hline & & $3 / 9 / 08$ & 13.1 & 7.09 & 758 & 5.95 & 212.9 \\
\hline & & 7/9/08 & 18.6 & 7.07 & 848 & 18.1 & 56.9 \\
\hline & & $10 / 22 / 08$ & 13.3 & 7.06 & 824 & 5.28 & 97.9 \\
\hline \multirow[t]{5}{*}{ MW14S } & $108-118$ & $4 / 4 / 07$ & 13.4 & 6.50 & 704 & - & - \\
\hline & & $11 / 18 / 07$ & 12.9 & 7.26 & 966 & - & - \\
\hline & & $3 / 8 / 08$ & 13.2 & 7.20 & 729 & 6.59 & 207.8 \\
\hline & & 7/10/08 & 17.4 & 7.16 & 775 & 16.4 & 86.7 \\
\hline & & $10 / 22 / 08$ & 13.2 & 7.11 & 716 & 5.81 & 90.2 \\
\hline \multirow{5}{*}{ MW14D } & $123-133$ & $4 / 4 / 07$ & 14.7 & 6.34 & 932 & - & - \\
\hline & & $11 / 18 / 07$ & 13.2 & 7.47 & 739 & - & - \\
\hline & & $3 / 8 / 08$ & 12.0 & 7.06 & 1424 & 1.95 & 282.4 \\
\hline & & $7 / 10 / 08$ & 17.7 & 7.07 & 1459 & 14.4 & 85.7 \\
\hline & & $10 / 22 / 08$ & 13.0 & 7.00 & 1212 & 1.41 & 78.7 \\
\hline
\end{tabular}


TABLE A.2 (Cont.)

\begin{tabular}{|c|c|c|c|c|c|c|c|}
\hline Well & $\begin{array}{l}\text { Screen Interval } \\
\quad \text { (ft BGL) }\end{array}$ & $\begin{array}{l}\text { Sample } \\
\text { Date }\end{array}$ & $\begin{array}{c}\text { Temperature } \\
\left({ }^{\circ} \mathrm{C}\right)\end{array}$ & $\mathrm{pH}$ & $\begin{array}{l}\text { Conductivity } \\
(\mu \mathrm{S} / \mathrm{cm})\end{array}$ & $\begin{array}{c}\text { Dissolved } \\
\text { Oxygen } \\
\text { (mg/L) }\end{array}$ & $\begin{array}{l}\text { Oxygen } \\
\text { Reduction } \\
\text { Potential } \\
(\mathrm{mV})\end{array}$ \\
\hline \multicolumn{8}{|c|}{ CCC/USDA wells installed during 2006-2007 investigation (cont.) } \\
\hline \multirow[t]{5}{*}{ MW15S } & $88-98$ & $4 / 4 / 07$ & 13.1 & 8.03 & 854 & - & - \\
\hline & & 11/18/07 & 13.9 & - & 1883 & - & - \\
\hline & & 3/10/08 & 12.1 & 8.67 & 697 & 5.49 & 173.2 \\
\hline & & 7/12/08 & 14.0 & 8.88 & 660 & 11.6 & 93.7 \\
\hline & & $10 / 23 / 08$ & 13.0 & 8.00 & 789 & 5.27 & 66.5 \\
\hline \multirow{5}{*}{ MW15D } & $105-115$ & $4 / 4 / 07$ & 14.8 & 6.15 & 2980 & - & - \\
\hline & & 11/18/07 & 13.1 & 6.85 & 2190 & - & - \\
\hline & & $3 / 8 / 08$ & 9.0 & 6.85 & 2912 & 0.57 & 130.6 \\
\hline & & $7 / 12 / 08$ & 14.1 & 6.80 & 3067 & 1.05 & 89.6 \\
\hline & & $10 / 24 / 08$ & 13.0 & 6.81 & 2876 & 0.34 & 27.0 \\
\hline \multirow[t]{5}{*}{ MW16S } & $76-86$ & $4 / 4 / 07$ & 12.8 & 6.35 & 1708 & - & - \\
\hline & & 11/19/07 & 15.0 & 6.94 & 1616 & - & - \\
\hline & & 3/7/08 & 7.3 & 6.96 & 1968 & 3.45 & 183.5 \\
\hline & & 7/11/08 & 18.8 & 6.71 & 2883 & 1.13 & 52.0 \\
\hline & & 10/23/08 & 11.6 & 7.01 & 1350 & 6.01 & 136.2 \\
\hline \multirow{5}{*}{ MW16D } & $90-100$ & $4 / 4 / 07$ & 14.1 & 6.17 & 2910 & - & - \\
\hline & & 11/19/07 & 12.5 & 6.78 & 2400 & - & - \\
\hline & & $3 / 7 / 08$ & 7.0 & 6.86 & 2866 & 0.50 & 140.0 \\
\hline & & 7/11/08 & 18.9 & 6.64 & 3134 & 0.41 & 31.5 \\
\hline & & 10/23/08 & 11.3 & 6.79 & 2791 & 0.45 & 37.4 \\
\hline \multirow[t]{5}{*}{ MW17 } & $120-130$ & $4 / 4 / 07$ & 16.0 & 6.44 & 861 & - & - \\
\hline & & 11/19/07 & 8.3 & 7.15 & 610 & - & - \\
\hline & & 3/5/08 & 5.5 & 7.12 & 804 & 7.02 & 239.4 \\
\hline & & $7 / 9 / 08$ & 17.5 & 7.11 & 843 & 20.6 & 89.4 \\
\hline & & $10 / 22 / 08$ & 13.1 & 7.10 & 777 & 8.35 & 109.7 \\
\hline \multicolumn{8}{|c|}{ Private wells } \\
\hline \multirow[t]{7}{*}{ Oentrich } & 150 & $7 / 20 / 06$ & - & - & - & - & - \\
\hline & & $8 / 2 / 06$ & - & - & - & - & - \\
\hline & & $4 / 5 / 07$ & - & - & - & - & - \\
\hline & & 11/19/07 & 12.1 & 8.26 & 1830 & - & - \\
\hline & & $3 / 6 / 08$ & - & - & - & - & - \\
\hline & & 7/11/08 & - & - & - & - & - \\
\hline & & $10 / 23 / 08$ & - & - & - & - & - \\
\hline \multirow[t]{2}{*}{ Sedivy } & 138 & $8 / 22 / 06$ & - & - & - & - & - \\
\hline & & 9/13/06 & 22.5 & 6.57 & 739 & - & - \\
\hline
\end{tabular}

a Not sampled; well dry.

b Questionable result. Value climbed steadily from 7.91 to 11.17 during the purging. 


\section{Appendix B:}

\section{Analytical Results for Wastewater Samples from Pace Analytical Services, Inc.}




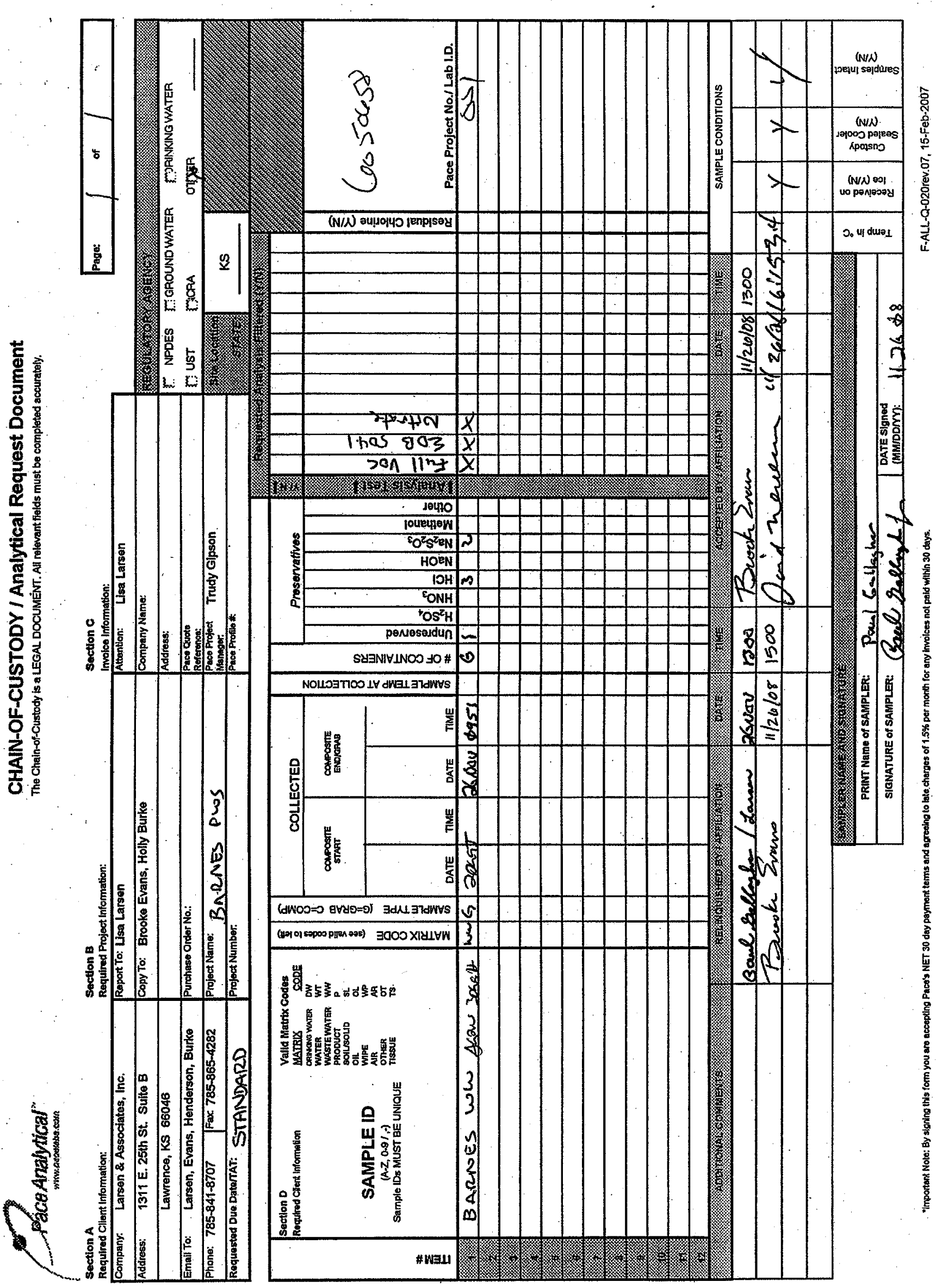


PaceAnalytical

Client Name:

Courier: $\square$ Fed Ex $\square$ uPs $\square$ USPS GClient $\square$ Commercial

Tracking \#:

Custody Seal on Cooler/Box Present: Gyes $\square$ no. Seals intact: 4 yes $\square$ no

Packing Material: $\square$ Bubble Wrap $\square$ bubble Bags $\square$ None $\triangle$ other for

Thermometer Used T-142y T-184

Cooler Temperature $\quad 3,4$

Temp should be above freezing to $6^{\circ} \mathrm{C}$

Chain of Custody Present:

Chain of Custody Filled Out:

Chain of Custody Relinquished:

Sampler Name \& Signature on $\mathrm{COC}$ :

Samples Arrived within Hold Time:

Short Hold Time Analysis ( $<72 \mathrm{hr})$ :

Rush Turn Around Time Requested:

Sufficient Volume:

Correct Containers Used:

-Pace Containers Used:

Containers intact:

Filtered volume received for Dissolved tests

Sample Labels match COC:

-Includes date/time/ID/Analysis

Matrix: $4 \mathrm{~J}$

All containers needing preservation have been checked.

All containers needing preservation are found to be in compliance with EPA recommendation.

exceptions: VøA, colfform, TOC, O\&G, wi-DRO (water)

Samples checked for dechlorination:

Headspace in VOA Vials $(>6 \mathrm{~mm})$ :

Trip Blank Present:

Trip Blank Custody Seals Present

Pace Trip Blank Lot \# (if purchased):

\section{Cllent Notification/ Resolution:}

Person Contacted:

Comments/ Resolution:
Project \# C Cos 6658

\section{Optional}

Proj. Due Date:

Proj. Name: $/ 2 / 10$
Biological Tissue is Frozen: Yos No

Comments:
Samples on ice, cooling process has begun Date and Initials of person examining contents:__olitalar Gres DNo DNA 1 . GYes 口No DNA 2. Gyes DNO DNA 3. GYes [No DN/A 4. $G_{\text {Yes }}$ 口No QNA 5. DYos ÉNo DN/A 6. 口Yes GNo INAA 7. Gyos पno DNa 8.

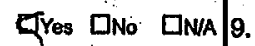
Eves QNo DNA Q DYes DNo Dina 11.

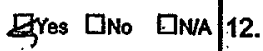
ares DNo GNA 13.

QYes पno \&ina

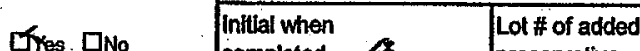

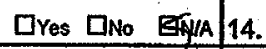
口Yes Q ZNo DNA 15.

DYes Zू/No DN/A 16.

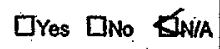
completed \& preservalive

Comments.

Date:

Note: Whenever there is a discrepancy affecting North Carolina compliance samples, a copy of this form will be sent to the North Carolina DEHNR Certification Office ( lie out of hold, incorrect preservative, out of temp, incorrect containers) 
December 10, 2008

Lisa Larsen

Larsen \& Associates, Inc.

1311 E. 25th St.

Suite B

Lawrence, KS 66046

RE: Project: BARNES PWS

Pace Project No.: 6050658

Dear Lisa Larsen:

Enclosed are the analytical results for sample(s) received by the laboratory on November 26, 2008. The results relate only to the samples included in this report. Results reported herein conform to the most current NELAC standards, where applicable, unless otherwise narrated in the body of the report.

If you have any questions concerning this report, please feel free to contact me.

Sincerely,

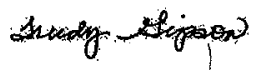

Trudy Gipson

trudy.gipson@pacelabs.com

Project Manager

Enclosures

cc: Holly Burke, Larsen \& Associates, Inc.

Brooke Evans, Larsen \& Associates, Inc. 
Pace Analytical Services, lnc. 9608 Loiret Blvd. Lenexa, KS 66219 (913)599-5665

\section{CERTIFICATIONS}

Project: BARNES PWS

Pace Project No.: 6050658

Kansas Certification IDs

Utah Certification Number: 9135995665

Texas Certification Number: T104704407-08-TX

Oklahoma Certification Number: $9205 / 9935$

Nevada Certification Number: KS000212008A

lowa Certification Number: 118

Illinois Certification Number: 001191

Arkansas Certification Number: 05-008-0.

A2LA Certification Number: 2456.01

Louisiana Certification Number: 03055

Kansas/NELAP Certification Number: E-10116

This report shall not be reproduced, except in full, without the written consent of Pace Analytical Services, Inc. 
Pace Analytical Services, Inc. 9608 Loiret Bivd. Lenexa, KS 66219

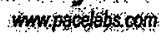
(913)599-5665

SAMPLE SUMMARY

Project: BARNES PWS

Pace Project No.: 6050658

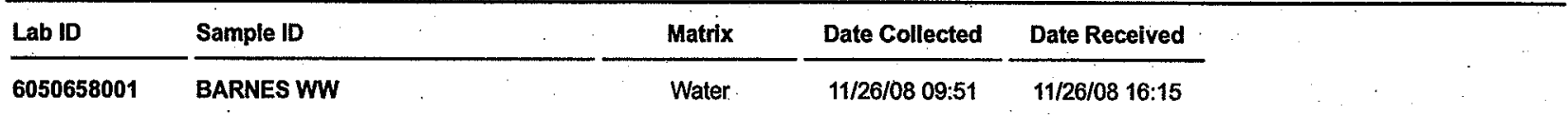




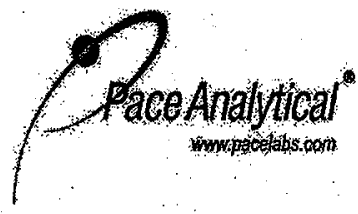

Pace Analytical Services, Inc. 9608 Loiret Bivd. Lenexa, KS 66219 (913)599-5665

\section{SAMPLE ANALYTE COUNT}

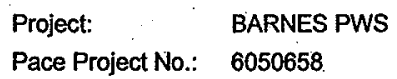

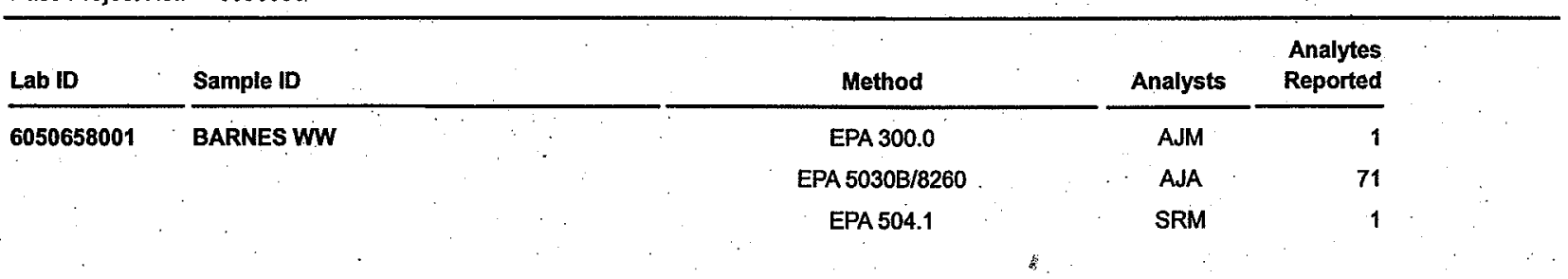

This report shall not be reproduced, except in full, without the written consent of Pace Analytical Services, inc.. sinela 


\section{ANALYTICAL RESULTS}

Project: BARNES PWS

Pace Project No.: 6050658

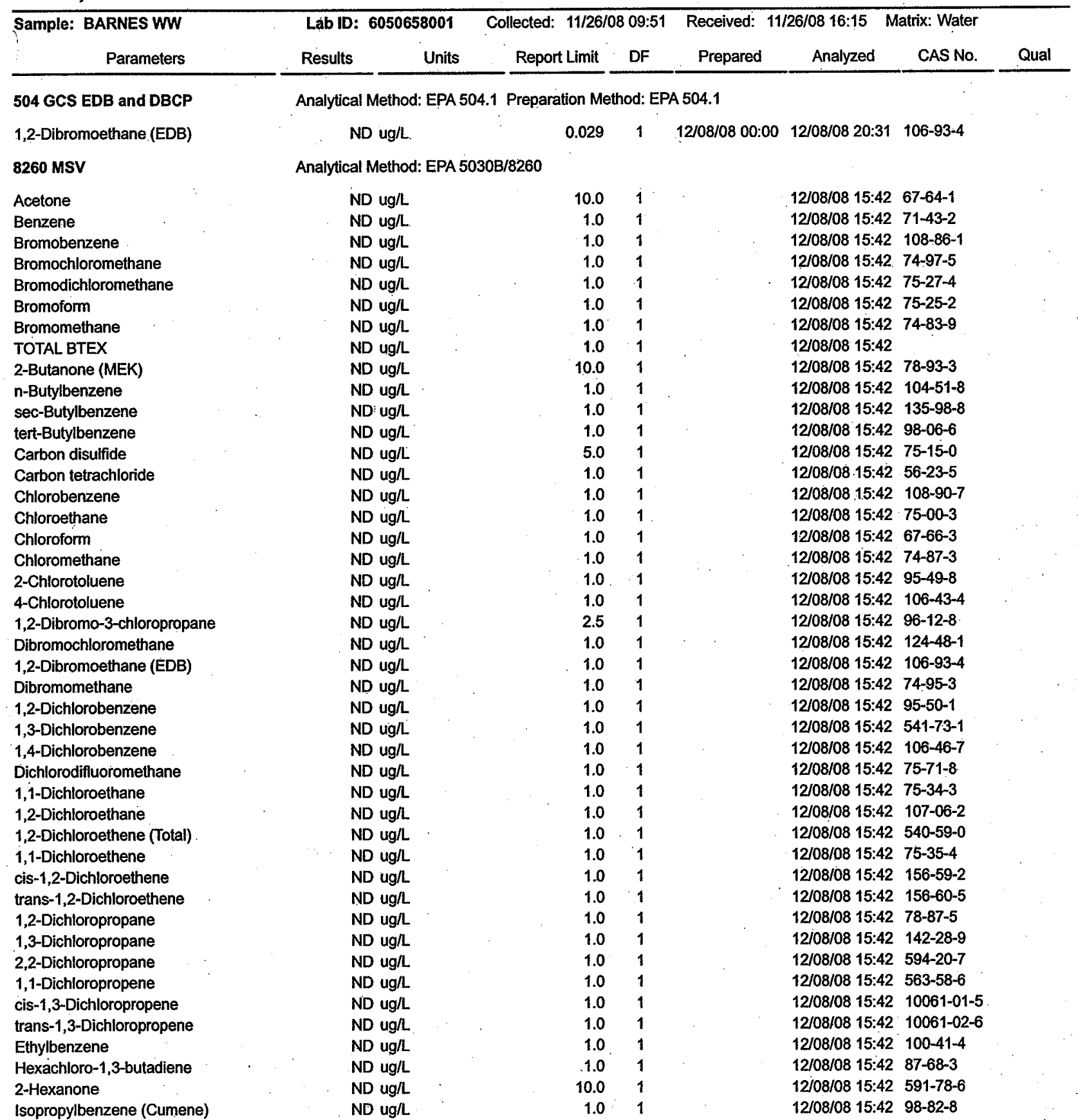

\section{REPORT OF' LABORATORY ANALYSIS}

This report shall not be reproduced, except in full,

without the written consent of Pace Analytical Services, Inc. 


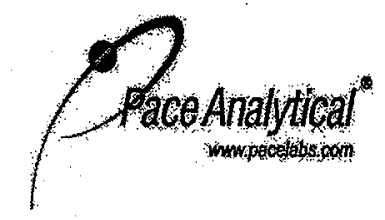

Pace Analytical Services, Inc. 9608 Loiret Blvd. Lenexa; KS 66219 (913)599-5665

\section{ANALYTICAL RESULTS}

Project: BARNES PWS

Pace Project No.: 6050658

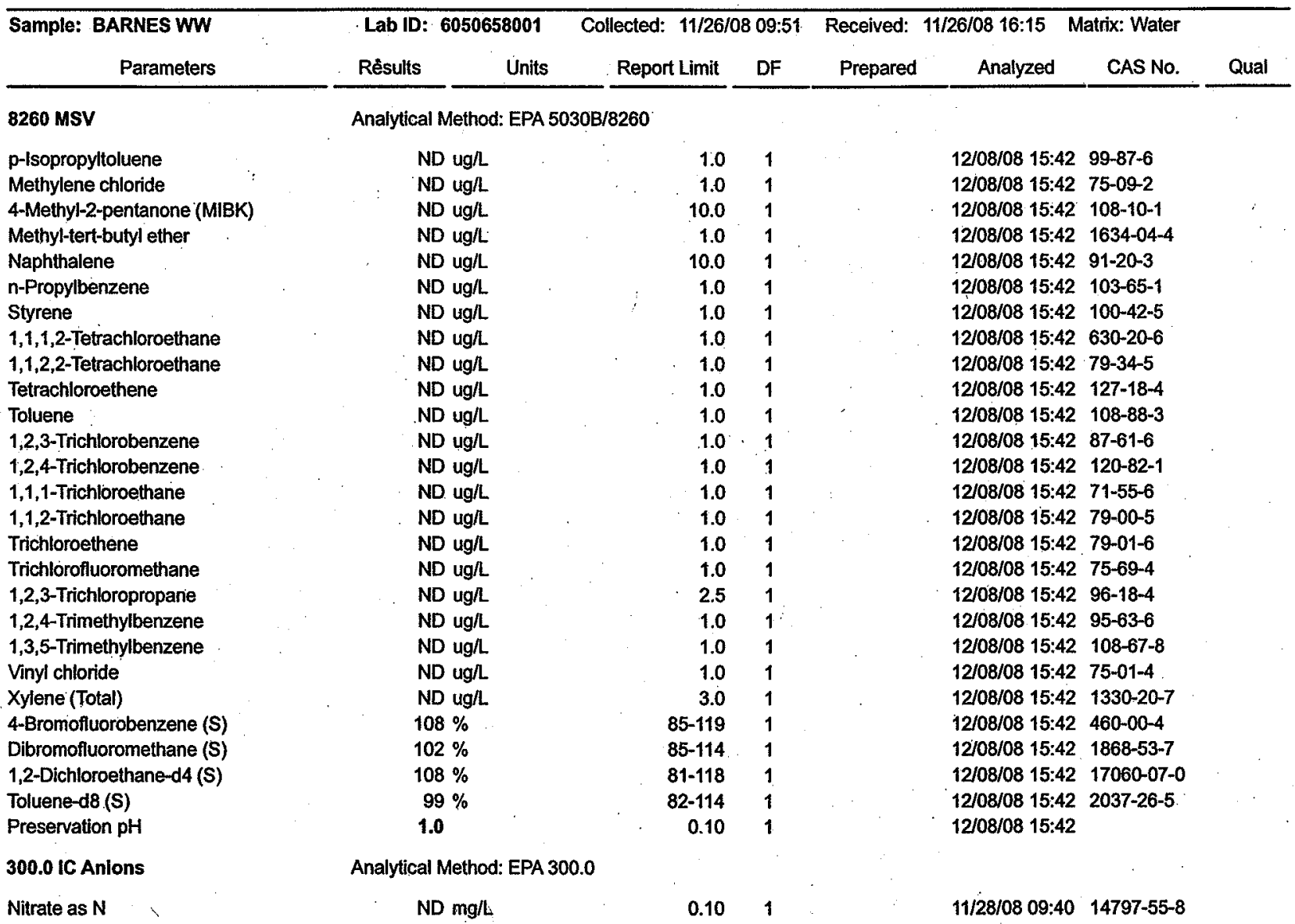




\section{QUALITY CONTROL DATA}

\section{Project: BARNES PWS \\ Pace Project No.: 6050658}

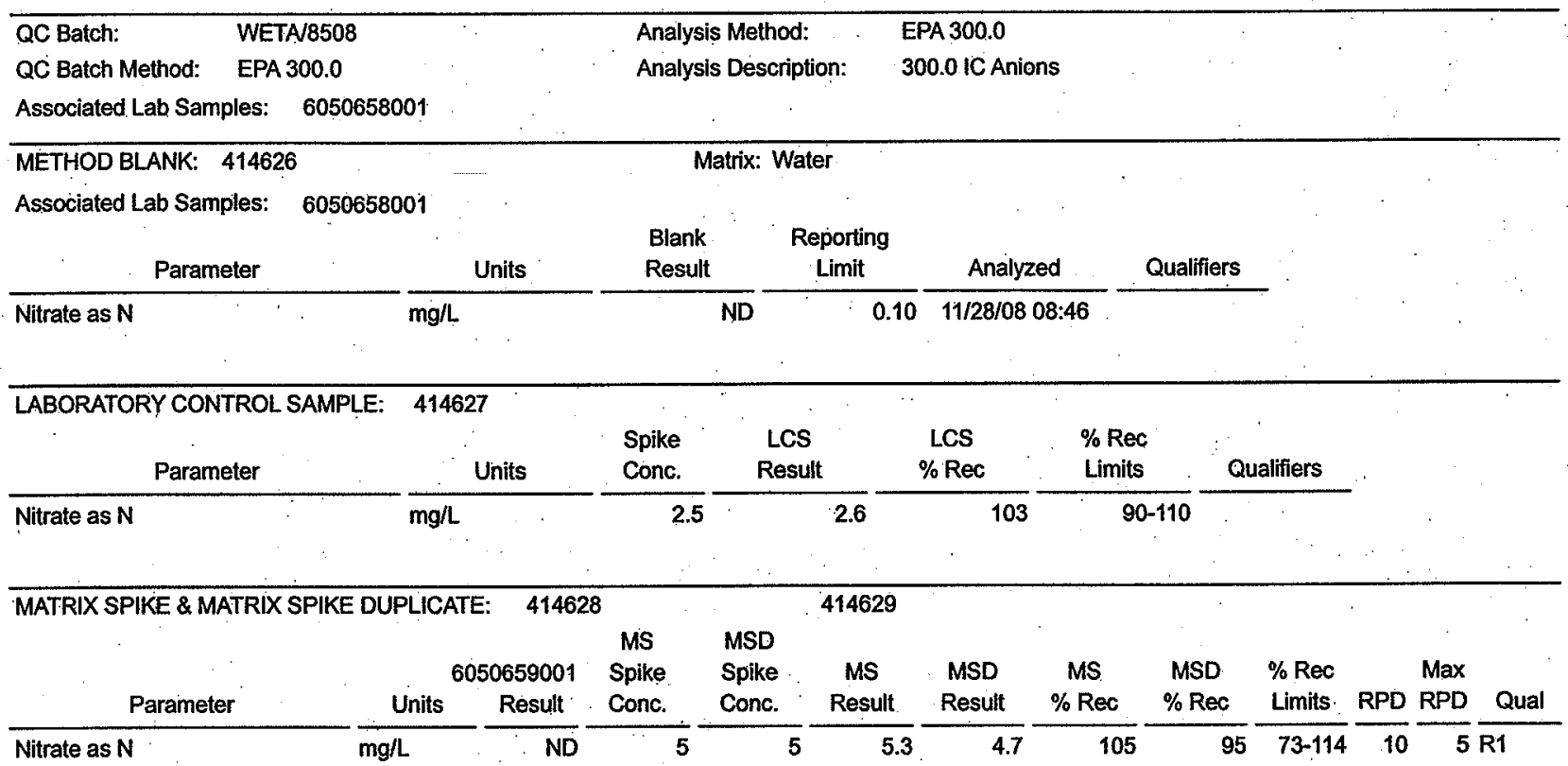




\section{QUALITY CONTROL DATA}

Project: $\quad$ BARNES PWS

Pace Project No.: $\quad 6050658$

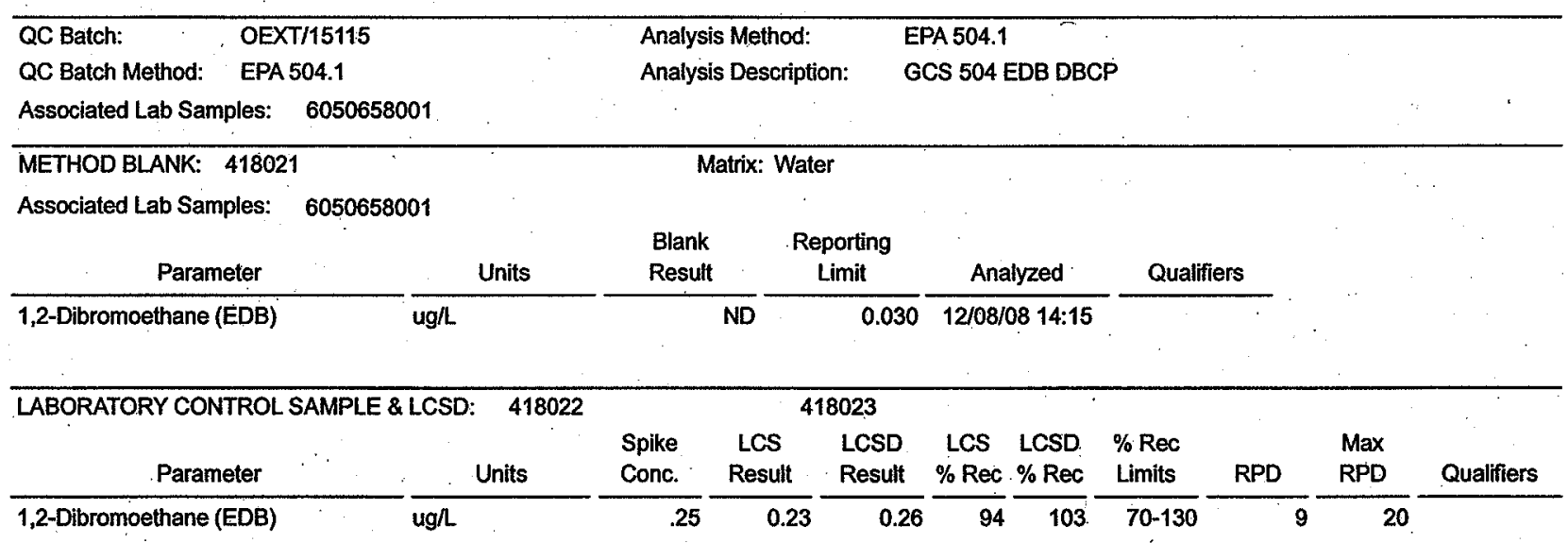


QUALITY CONTROL DATA

Project: BARNES PWS

Pace Project No.: 6050658

\begin{tabular}{|c|c|c|c|}
\hline QC Batch: & MSV/18481 & Analysis Method: & EPA 5030B/8260 \\
\hline QC Batch Method: & $\mathrm{EPA} 5030 \mathrm{~B} / 8260$ & Analysis Description: & 8260 MSV Water $10 \mathrm{~mL}$ Purge \\
\hline
\end{tabular}

METHOD BLANK: 418050

Associated Lab Samples: $\quad 6050658001$

\section{Parameter}

1,1,1,2-Tetrachloroethane

1,1,1-Trichloroethane

1,1,2,2-Tetrachloroethane

1,1,2-Trichloroethane

1,1-Dichloroethane

1,1-Dichloroethene

1,1-Dichloropropene

1,2,3-Trichlorobenzene

1,2,3-Trichloropropane

1,2,4-Trichlorobenzene

1,2,4-Trimethylbenzene

1,2-Dibromo-3-chloropropane

1,2-Dibromoethane (EDB)

1,2-Dichlorobenzene

1,2-Dichloroethane

1,2-Dichloroethene (Total)

1,2-Dichloropropane

1,3,5-Trimethylbenzene

1,3-Dichlorobenzene

1,3-Dichloropropane

1,4-Dichlorobenzene

2,2-Dichloropropane

2-Butanone (MEK)

2-Chlorotoluene

2-Hexanone

4-Chlorotoluene

4-Methyl-2-pentanone (MIBK)

Acetone

Benzene

Bromobenzene

Bromochloromethane

Bromodichloromethane

Bromoform

Bromomethane

Carbon disutide

Carbon tetrachioride

Chlorobenzene

Chloroethane

Chloroform

Chloromethane

cis-1,2-Dichloroethene

cis-1,3-Dichloropropene

Dibromochloromethane

Date: 12/10/2008 03:01 PM
Matrix: Water

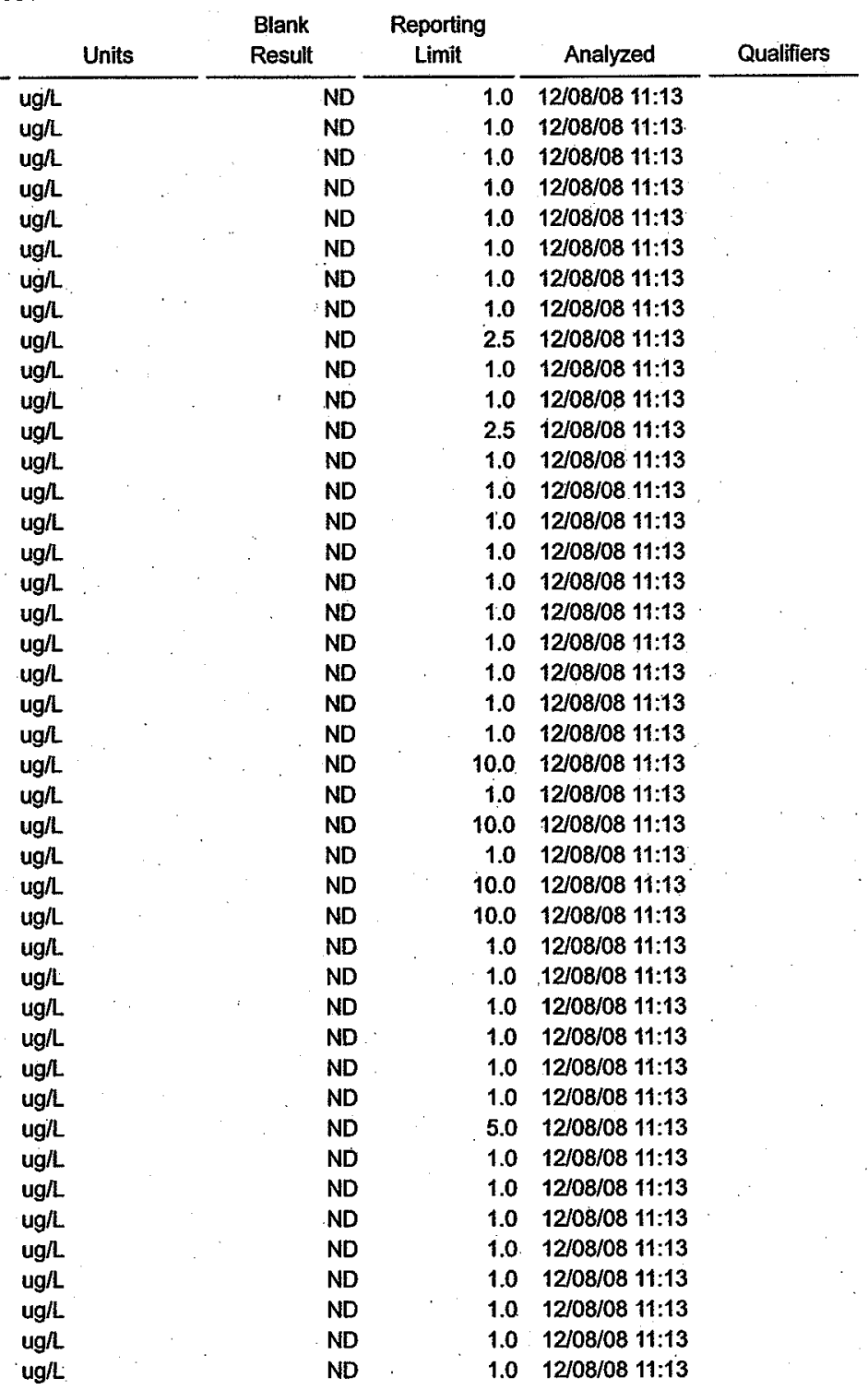

\section{REPORT OF LABORATORY ANALYSIS}

This report shall not be reproduced, except in full, without the written consent of Pace Analytical Services, Inc.. 


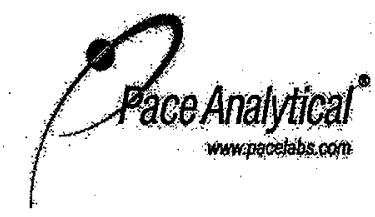

Pace Analytical Services, Inc. 9608 Loiret Bivd. Lenexa, KS 66219 (913)599-5665

\section{QUALITY CONTROL DATA}

Project: $\quad$ BARNES PWS

Pace Project No.: 6050658

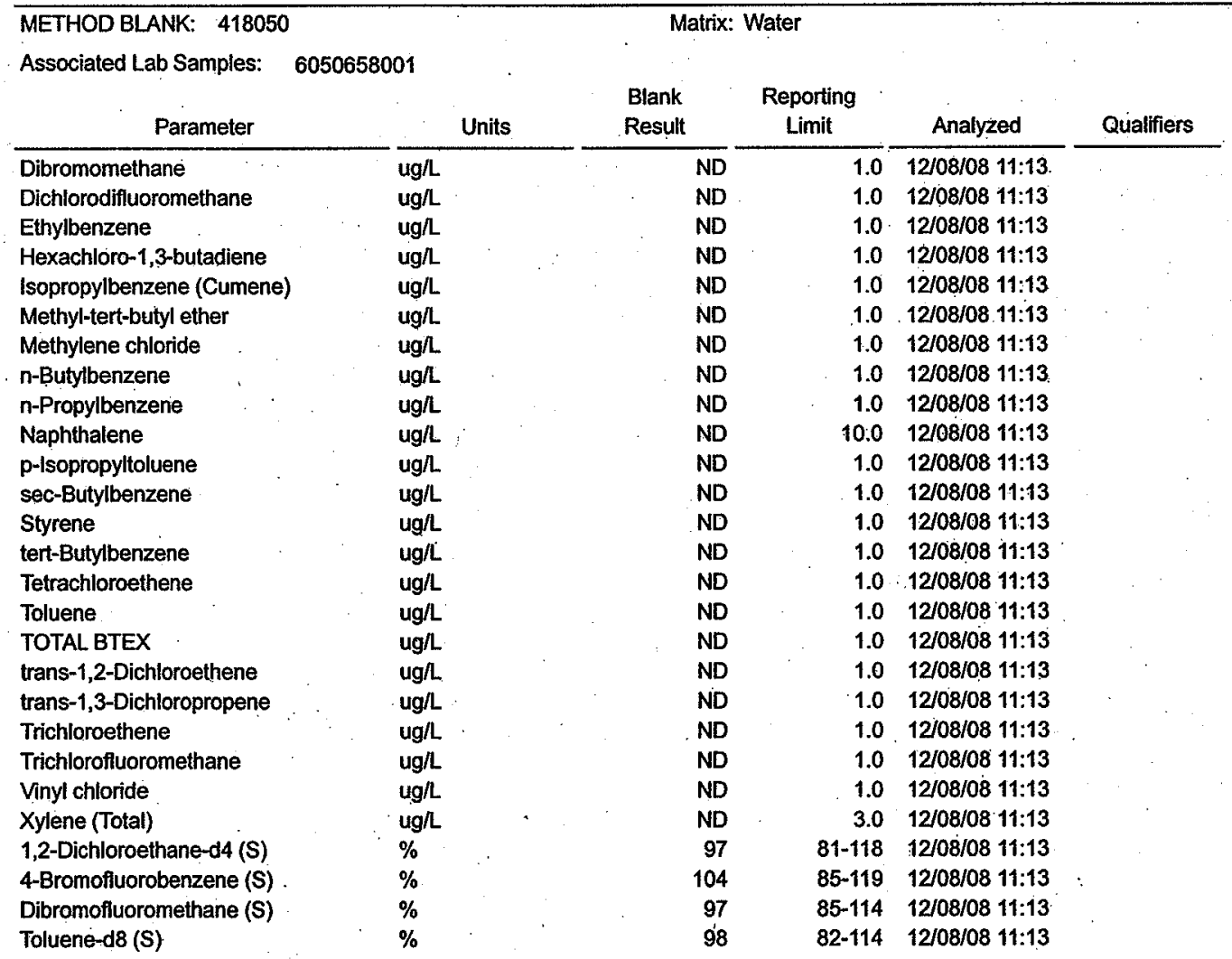

\begin{tabular}{|c|c|c|c|c|c|c|}
\hline \multirow{2}{*}{$\begin{array}{c}\text { LABORATORY CONTROL SAMPLE: } \\
\text { Parameter }\end{array}$} & \multicolumn{6}{|l|}{418051} \\
\hline & Units. & $\begin{array}{l}\text { Spike } \\
\text { Conc. }\end{array}$ & $\begin{array}{c}\text { LCS } \\
\text { Result }\end{array}$ & $\begin{array}{l}\text { LCS } \\
\% \operatorname{ReC}\end{array}$ & $\begin{array}{l}\% \operatorname{Rec} \\
\text { Limits }\end{array}$ & Qualifiers \\
\hline 1,1,1,2-Tetrachloroethane & $\overline{u g} / \mathrm{L}$ & 10 & 10.9 & 109 & $77-127$ & $\because$ \\
\hline 1,1,1-Trichloroethane & $u g / L$ & 10 & 10.6 & 106 & $78-130$ & \\
\hline 1,1,2,2-Tetrachloroethane & $\mathrm{ug} / \mathrm{L}$ & 10 & 10.2 & 102 & $73-131$ & \\
\hline 1,1,2-Trichloroethane & ug/L & 10 & 11.2 & 112 & $85-126$ & \\
\hline 1,1-Dichloroethane & $u g / L$ & 10 & 10.7 & 107 & $76-124$ & \\
\hline 1,1-Dichloroethene & $\mathrm{ug} / \mathrm{L}$ & 10 & 10.8 & 108 & $76-129$ & \\
\hline 1,1-Dichloropropene & ug/L & 10 & 11.1 & 111 & $83-125$ & \\
\hline 1,2,3-Trichlorobenzene & $\mathrm{ug} / \mathrm{L}$ & 10 & 11.6 & 116 & $78-129$ & \\
\hline 1,2,3-Trichloropropane & $u g / L$ & 10 & 11.0 & 110 & $69-117$ & \\
\hline 1,2,4-Trichlorobenzene & ug/L & 10 & 11.6 & 116 & $79-127$ & \\
\hline 1,2,4-Trimethylbenzene & ug/L & 10 & 10.3 & 103 & $82-124$ & \\
\hline 1,2-Dibromo-3-chloropropane & $\mathrm{ug} / \mathrm{L}$ & 10 & 13.0 & 130 & $62-141$ & \\
\hline 1,2-Dibromoethane (EDB). & $u g / L$ & 10 & 10.9 & 109 & $85-124$ & \\
\hline 1,2-Dichlorobenzene & $u g / L$ & 10 & 9.2 & 92 & $85-123$ & \\
\hline
\end{tabular}

Date: 12/10/2008 03:01 PM

REPORT OF LABORATORY ANALYSIS

Page 10 of 14

This report shall not be reproduced, except in full,

without the written consent of Pace Analytical Services, Inc.

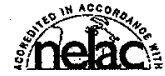




\section{QUALITY CONTROL DATA}

Project:

BARNES PWS

Pace Project No.: 6050658

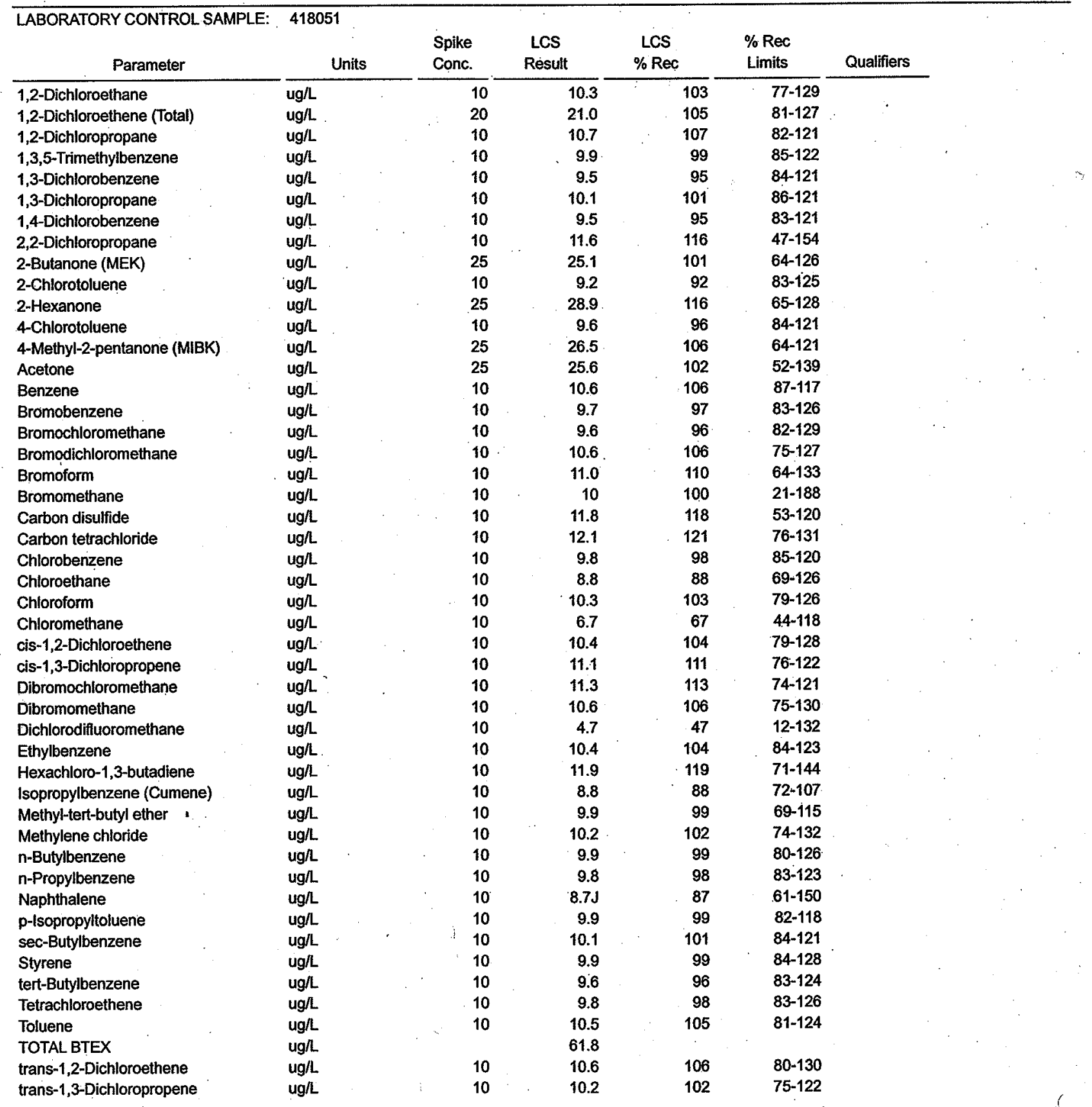

Date: 12/10/2008 03:01 PM 


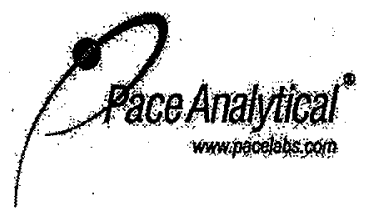

Pace Analytical Services, Inc.

9608 Loiret Blvd.

Lenexa, KS 66219

(913)599-5665

QUALITY CONTROL DATA

$\begin{array}{ll}\text { Project: } & \text { BARNES PWS } \\ \text { Pace Project No: } & 6050658\end{array}$

LABORATORY CONTROL SAMPLE: 418051

\begin{tabular}{|c|c|c|c|c|c|c|}
\hline Parameter & & $\begin{array}{l}\text { Spike } \\
\text { Conc. }\end{array}$ & $\begin{array}{c}\text { LCS } \\
\text { Result }\end{array}$ & $\begin{array}{c}\text { LCS } \\
\% \text { Rec }\end{array}$ & $\begin{array}{l}\% \text { Rec } \\
\text { Limits }\end{array}$ & Qualifiers \\
\hline Trichloroethene & ug/L & 10 & 10.6 & 106 & $80-130$ & \\
\hline Trichlorofluoromethane & ug $/ \mathrm{L}$ & 10 & 9.7 & 97 & $65-113$ & \\
\hline Vinyl chleride & ug/L & 10 & 9.7 & 97. & $59-124$ & \\
\hline Xylene (Total) & ug/L & 30 & 30.3 & 101 & $83-125$ & \\
\hline 1,2-Dichloroethane-d4 (S) & $\%$ & & & 106 & $81-118$ & \\
\hline 4-Bromofluorobenzene (S) & $\%$ & & & 102 & $85-119$ & \\
\hline Dibromofluoromethane (S) & $\%$ & & & 101 & $85-114$ & \\
\hline Toluene-d8 (S) & $\%$ & : & & 101 & $.82-114$ & \\
\hline
\end{tabular}




\title{
QUALIFIERS
}

\author{
Project: $\quad$ BARNES PWS \\ Pace Project No.: $\quad 6050658$
}

\section{DEFINITIONS}

DF - Dilution Factor, if reported, represents the factor applied to the reported data due to changes in sample preparation, dilution of the sample aliquot, or moisture content.

ND - Not Detected at or above adjusted reporting limit.

$\mathrm{J}$ - Estimated concentration above the adjusted method detection limit and below the adjusted reporting limit.

MDL - Adjusted Method Detection Limit.

S - Surrogate

1,2-Diphenylhydrazine ( 8270 listed analyte) decomposés to Azobenzene.

Consistent with EPA guidelines, unrounded data are displayed and have been used to calculate \% recovery and RPD values.

LCS(D) - Laboratory Control Sample (Duplicate)

MS(D) - Matrix Spike (Duplicate)

DUP - Sample Duplicate

RPD - Relative Percent Difference

Pace Analytical is NELAP accredited. Contact your Pace PM for the current list of accredited analytes.

\section{BATCH QUALIFIERS}

Batch: MSV/18481

[1] A matrix spike/matrix spike duplicate was not performed for this batch due to insufficient sample volume.

\section{ANALYTE QUALIFIERS}

R1 RPD value was outside control limits. 


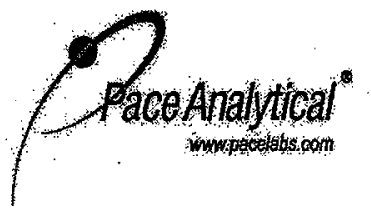

Pace Analytical Services, Inc. 9608 Loiret Blvd. Lenexa, KS 66219 (913)599-5665

QUALITY CONTROL DATA CROSS REFERENCE TABLE

$\begin{array}{ll}\text { Project: } & \text { BARNES PWS } \\ \text { Pace Project No.: } & 6050658\end{array}$

\begin{tabular}{|c|c|c|c|c|c|c|}
\hline Lab ID & Sample ID & & QC Batch Method & QC Batch & Analytical Method & $\begin{array}{l}\text { Analytical } \\
\text { Batch }\end{array}$ \\
\hline 6050658001 & BARNES WW & & EPA 300.0 & WETA/8508 & & \\
\hline 6050658001 & BARNES WW & . & EPA 504.1 & OEXT/15115 & EPA 504.1 & GCSV/6219 \\
\hline 6050658001 & BARNES WW & . & EPA $5030 \mathrm{~B} / 8260$ & MSV/18481 & . & \\
\hline
\end{tabular}


AppendixC:

Results from the AGEM Laboratory for Dual Analyses of Samples Collected at Barnes in October 2008 and for Quality Control Samples 
TABLE C.1 Analytical results for samples and replicates collected at Barnes in October 2008 and for quality control samples.

\begin{tabular}{|c|c|c|c|c|c|c|c|}
\hline \multirow[b]{2}{*}{$\begin{array}{l}\text { Sample } \\
\text { Date }\end{array}$} & \multirow[b]{2}{*}{ Location } & \multirow[b]{2}{*}{$\begin{array}{l}\text { Depth } \\
\text { (ft BGL) }\end{array}$} & \multirow[b]{2}{*}{ Sample } & \multirow[b]{2}{*}{ Analysis Type } & \multicolumn{3}{|c|}{ Concentration $(\mu \mathrm{g} / \mathrm{L})$} \\
\hline & & & & & $\begin{array}{c}\text { Carbon } \\
\text { Tetrachloride }\end{array}$ & Chloroform & $\begin{array}{c}\text { Methylene } \\
\text { Chloride }\end{array}$ \\
\hline 10/22/08 & MW1D & $139.85-159.4$ & BAMW1D-W-27720 & Primary & $N D^{a}$ & ND & ND \\
\hline $10 / 22 / 08$ & MW1D & $139.85-159.4$ & BAMW1DDUP-W-27750 & Replicate & ND & ND & ND \\
\hline 10/23/08 & MW10D & $115-125$ & BAMW10D-W-27731 & Primary & 4.4 & $0.6 \mathrm{~J}^{\mathrm{b}}$ & ND \\
\hline $10 / 23 / 08$ & MW10D & $115-125$ & BAMW10D-W-27731DUP & Duplicate & 4.5 & $0.5 \mathrm{~J}$ & ND \\
\hline 10/22/08 & MW13S & $112-122$ & BAMW13S-W-27738 & Primary & 20 & 1.6 & ND \\
\hline 10/22/08 & MW13S & $112-122$ & BAMW13SDUP-W-27751 & Replicate & 23 & 1.8 & ND \\
\hline 10/22/08 & MW17 & $120-130$ & BAMW17-W-27746 & Primary & $0.7 \mathrm{~J}$ & ND & ND \\
\hline 10/22/08 & MW17 & $120-130$ & BAMW17-W-27746DUP & Duplicate & $0.6 \mathrm{~J}$ & ND & ND \\
\hline 10/22/08 & QC & - & BAQCIR-W-27752 & Rinsate after MW13D & ND & ND & ND \\
\hline 10/22/08 & QC & - & BAQCIR-W-27753 & Rinsate after MW17 & ND & ND & ND \\
\hline 10/22/08 & QC & - & BAQCTB-W-27754 & Trip blank & ND & ND & ND \\
\hline $10 / 23 / 08$ & QC & - & BAQCTB-W-27755 & Trip blank & ND & ND & ND \\
\hline $10 / 24 / 08$ & $\mathrm{QC}$ & - & BAQCTB-W-27756 & Trip blank & ND & $0.4 \mathrm{~J}$ & ND \\
\hline
\end{tabular}

a ND, contaminant not detected at an instrument detection limit of $0.1 \mu \mathrm{g} / \mathrm{L}$.

${ }^{\mathrm{b}}$ Qualifier $\mathrm{J}$ indicates an estimated concentration below the method quantitation limit of $1.0 \mu \mathrm{g} / \mathrm{L}$. 


\section{Appendix D:}

Sample Documentation from TestAmerica Laboratories, Inc. 


\section{TestAmerica \\ THE LEADER IN ENVIRONMENTAL TESTING}

November 6, 2008

TestAmerica Laboratories, Inc.

Mr. Clyde Dennis

Argonne National Laboratory

9700 S. Cass Avenue

Building 203, Office B149

Argonne, IL 60439

Re: Laboratory Project No. 21005

Case: BARNES; SDG: 128393

Dear Mr. Dennis:

Enclosed are analytical results for samples that were received by TestAmerica Burlington on

October $24^{\text {th }}, 2008$. Laboratory identification numbers were assigned, and designated as follows:

$\begin{array}{llll}\text { Lab ID } & \begin{array}{l}\text { Client } \\ \text { Sample ID }\end{array} & \begin{array}{l}\text { Sample } \\ \text { Date }\end{array} & \begin{array}{l}\text { Sample } \\ \text { Matrix }\end{array} \\ & \begin{array}{llll}\text { Received: 10/24/08 ETR No: } \\ 128393\end{array} & \\ 772906 & \text { BAMW3D-W-27722 } & 10 / 22 / 08 & \text { WATER } \\ 772907 & \text { BAMW17-W-27746 } & 10 / 22 / 08 & \text { WATER } \\ 772908 & \text { BAMW7-W-27727 } & 10 / 23 / 08 & \text { WATER } \\ 772909 & \text { BAMW11S-W-27732 } & 10 / 23 / 08 & \text { WATER } \\ 772910 & \text { BAPWS2-W-27748 } & 10 / 23 / 08 & \text { WATER } \\ 772911 & \text { BAQCTB-W-27755 } & 10 / 23 / 08 & \text { WATER } \\ 772912 & \text { VHBLK01 } & 10 / 24 / 08 & \text { WATER }\end{array}$

Documentation of the condition of the samples at the time of their receipt and any exception to the laboratory's Sample Acceptance Policy is documented in the Sample Handling section of this submittal. The samples, as received, were not acid preserved. On that basis the laboratory did provide for the analytical work to be performed within seven days of sample collection.

In order to accommodate field length limitations in processing the data summary forms, the laboratory did, in certain instances, abbreviate the sample identifier. The electronically formatted data provides for the full sample identifier.

\section{SOM01.2 Volatile Organics (Trace Level Water)}

A storage blank was prepared for volatile organics analysis, and stored in association with the

SDG: 128393

TestAmerica Burlington

Page 1.1 of 179

30 Community Drive Suite 11 South Burlington, VT 05403 tel 802.660.1990 fax 802.660.1919 www.testamericainc.com 


\section{TestAmerica}

THE LEADER IN ENVIRONMENTAL TESTING

storage of the samples. That storage blank, identified as VHBLK01, was carried through the holding period with the samples, and analyzed.

Each of the analyses associated with the sample set exhibited an acceptable internal standard performance. There was an acceptable recovery of each deuterated monitoring compound (DMC) in the analysis of the method blank associated with the analytical work, and in the analysis of the storage blank associated with the sample set. The analysis of the samples in this sample set did meet the technical acceptance criteria specific to DMC recoveries, although not all DMC recoveries were within the control range in each analysis. The technical acceptance criteria does provide for the recovery of up to three DMCs to fall outside of the control range in the analysis of field samples. The recoveries of 2-butanone- $d_{5}$ and 2 -hexanone- $d_{5}$. were elevated in the analysis of several of the field samples. Matrix spike and matrix spike duplicate analyses were not performed on samples in this sample set. A trace concentration of acetone was identified in the analysis of the method blank associated with the analytical work. The concentration of acetone in that analysis was below the established reporting limit, and the analysis did meet the technical acceptance criteria for a compliant method blank analysis. A trace concentration of acetone was identified in the analysis of the storage blank associated with the sample set. The concentration of acetone in that analysis was below the established reporting limit, and the analysis did meet the technical acceptance criteria for a compliant storage blank analysis. Present in the method blank and storage blank analyses was a non-target constituent that represents a compound that is related to the DMC formulation. The fact that the presence of this compound is not within the laboratory's control is at issue. The derived results for that compound have been qualified with an " $\mathrm{X}$ " qualifier to refiect the source of the contamination.

The responses for each target analyte met the relative standard deviation criterion in each initial calibration. The response for each target analyte met the percent difference criterion in the continuing calibration check acquisition. The response for each target analyte met the 50.0 percent difference criterion in the closing calibration check acquisition.

The primary quantitation mass for methylcyclohexane that is specified in the Statement of Work is mass 83 . The laboratory did identify a contribution to mass 83 from 1,2-dichloropropane-d. one of the deuterated monitoring compounds (DMCs). The laboratory did change the primary quantitation mass assignment to mass 55 for the quantification of methylcyclohexane.

Manual integration was employed in deriving certain of the analytical results. The values that have been derived from manual integration are qualified on the quantitation reports. Extracted ion current profiles for each manual integration are included in the data package, and further documented in the Sample Preparation section of this submittal.

Any reference within this report to Severn Trent Laboratories, Inc. or STL, should be understood to refer to TestAmerica Laboratories, Inc. (formerly known as Severn Trent Laboratories, Inc.) The analytical results associated with the samples presented in this test report were generated under a quality system that adheres to requirements specified in the NELAC standard. Release of the data in this test report and any associated electronic deliverables is authorized by the Laboratory Director's designee as verified by the following signature. 


\section{TestAmerica \\ THE LEADER IN ENVIRONMENTAL TESTING}

If there are any questions regarding this submittal, please contact me at 802 660-1990.

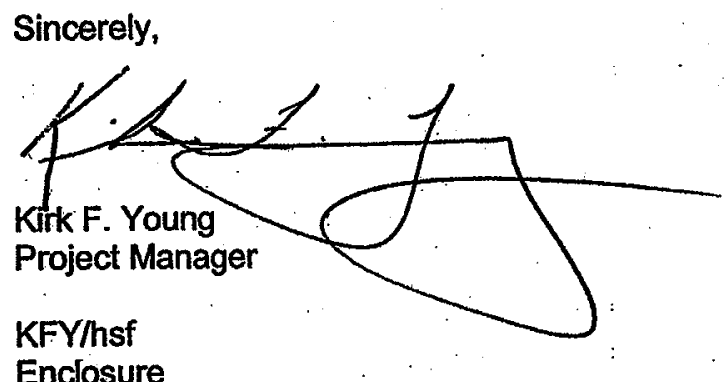

Enclosure 


\section{TestAmerica Burlington Data Qualifier Definitions}

\section{Organic}

U: Compound analyzed but not detected at a concentration above the reporting limit.

J: Estimated value.

N: Indicates presumptive evidence of a compound. This flag is used only for tentatively identified compounds (TICS) where the identification of a compound is based on a mass spectral library search.

P: SW-846: The relative percent difference for detected concentrations between two GC columns is greater than $40 \%$. Unless otherwise specified the higher of the two values is reported on the Form $l$.

CLP SOW: Greater than $25 \%$ difference for detected concentrations between two GC columns. Unless otherwise specified the lower of the two values is reported on the Form I.

C: Pesticide result whose identification has been confirmied by GCMS.

B: Analyte is found in the sample and the associated method blank. The flag is used for tentatively identified compounds as well as positively identified compounds.

E: Compounds whose concentrations exceed the upper limit of the callibation range of the instrument for that specific analysis.

D: Concentrations identified from analysis of the sample at a secondary dilution.

A: Tentatively identified compound is a suspected aldol condensation product.

$X, Y, Z$ : Laboratory defined flags that may be used alone or combined, as needed. If used, the description of the flag is defined in the project narrative.

\section{Inorganic/Metals}

E: Reported value is estimated due to the presence of interference.

N: Matrix spike sample recovery is not within control limits.

- Duplicate sample analysis is not within control limits.

B: The result reported is less than the reporting limit but greater than the instrument detection limit.

U: Analyte was analyzed for but not detected above the reporting limit.

Method Codes:

P ICPAES

MS ICP-MS

CV Cold Vapor AA

AS Semi-Automated Spectrophotometric

FQA009:02.18.0844

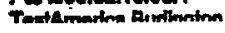




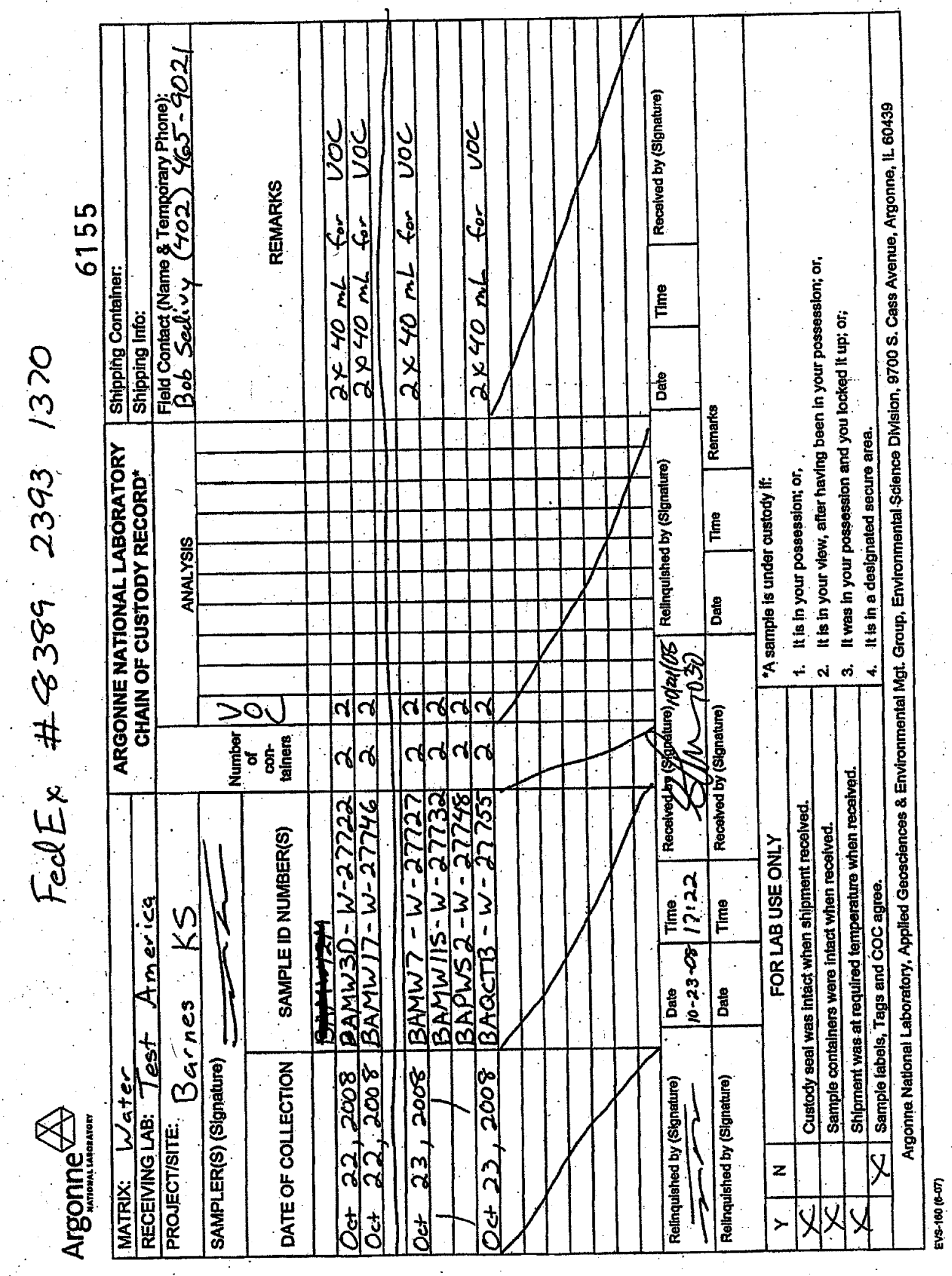

SDG: 128393

TestAmerica Burlington

Page 4 of 179 


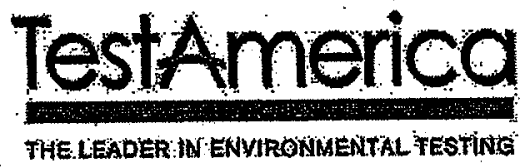

\section{Supportive Documentation - SOM01.2 \\ Volatiles - Trace}


IA - FORM I VOA-I

VOLATILE ORGANICS ANALYSIS DATA SHEET

Iab Name: TESTAMERICA BURLINGTON Lab Code: STLV Case No.: BARIES Matrix: (SOIL/SED/WATER) Water Sample wt/vol: 25.0 $(g / m L) \mathrm{mL}$

Level: (TRACE/LOW/MED) TRACE

\% Moisture: not dec.

GC Columin: $D B-624$

Soil Extract Volume:

ID: 0.53

(mm)

(uL)

Contract: 21005

Mod. Ref No.:

SDG NO.: 128393

Lab Sample ID: 772909

Lab File ID: 772909

Date Received: $10 / 24 / 2008$

Date Analyzed: $10 / 27 / 2008$

Dilution Factor: 1.0

Purge Volume: 25.0

(mL)

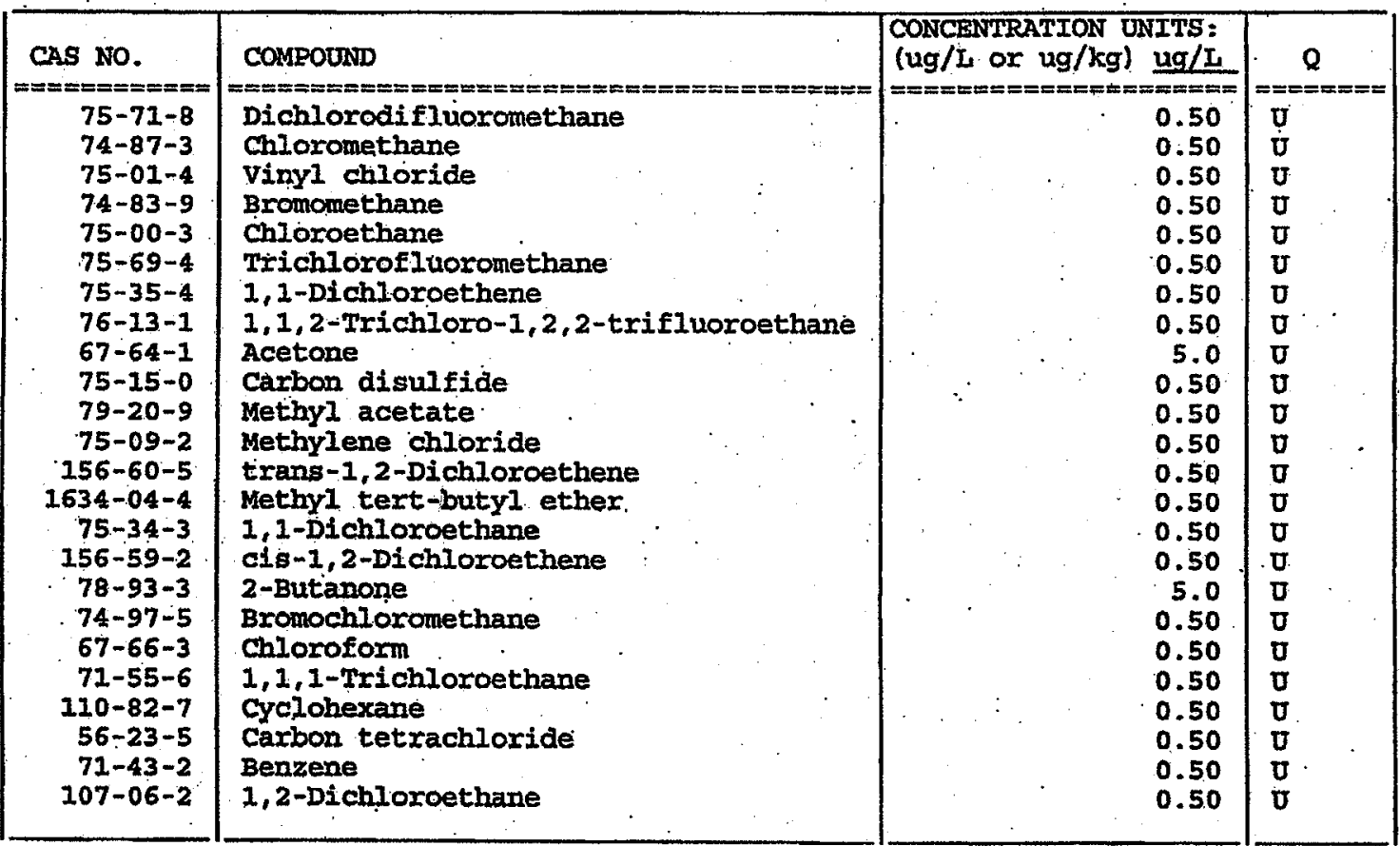

Report 1,4-Dioxane for LOW-Medium VOA analysis only

SOM01.2 
IB - FORM I VOA-2

VOLATILE ORGANICS ANALYSIS DATA SHEET

EPA SAMPLE NO.

Iab Name: TESTAMERICA BURIINGTON

Contract: 21005

Iab code: STLV Case No.: BARNES

Mod. Ref No.:

SDG NO.: 128393

Matrix: (SOIL/SED/WATER) Water

Sample wt/vol: $25.0 \quad(\mathrm{~g} / \mathrm{mL}) \mathrm{mL}$

Level: (TRACE/LOW/MED) TRACE

\%oisture: not dec.

GC Column: DB-624

Soil Extract Volume:

$I D: 0.53 \quad(\mathrm{~mm})$

(uIt)

(mL)

Purge volume: 25.0
Lab Sample ID: 772909

Iab File ID: 772909

Date Reçeived: $10 / 24 / 2008$

Date Analyzed: $10 / 27 / 2008$

Dilution Factor: 1.0

Soil aliquot volume:

(uIJ)

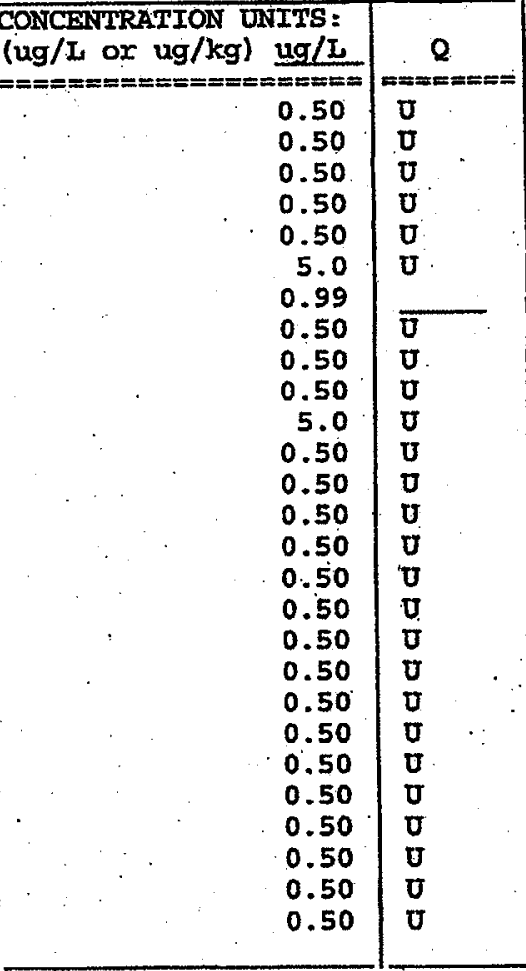

SOMO1.2 
$1 A$ - FORM I VOA-1

VOIATILE ORGANICS ANALYSIS DATA SHEET

EPA SAMPLE NO.

Lab Name: TESTAMERICA BURIINGTON

Contract: 21005

Lab Code: STLV Case No.: BARNES

Mod. Ref NTo.:

SDG NO.: 128393

Matrix: (SOIL/SED/KATER) Water

Lab Sample ID: $\mathbf{7 7 2 9 0 7}$

Sample wt/vol: $25.0 \quad(\mathrm{~g} / \mathrm{mL}) \mathrm{mL}$

Iab File ID: 772907

Level: (TRACE/IOW/MED) TRACE

Date Received: $10 / 24 / 2008$

: Moisture: not dec.

Date Analyzed: 10/27/2008

GC Column: DB-624

ID: 0.53

(ma)

Dilution Factor: 1.0

Soil Extract Volume:

(UL)

Soil Aliquot volume:

(uL)

Purge volume: 25.0

(mis)

\begin{tabular}{|c|c|c|c|c|}
\hline $\begin{array}{r}\text { CAS } 10 . \\
75-71-8 \\
74-87-3 \\
75-01-4 \\
74-83-9 \\
75-00-3 \\
75-69-4 \\
75-35-4 \\
76-13-1 \\
67-64-1 \\
75-1.5-0 \\
79-20-9 \\
75-09-2 \\
156-60-5 \\
1634-04-4 \\
75-34-3 \\
156-59-2 \\
78-93-3 \\
74-97-5 \\
67-66-3 \\
71-55-6 \\
110-82-7 \\
56-23-5 \\
71-43-2 \\
107-06-2\end{array}$ & 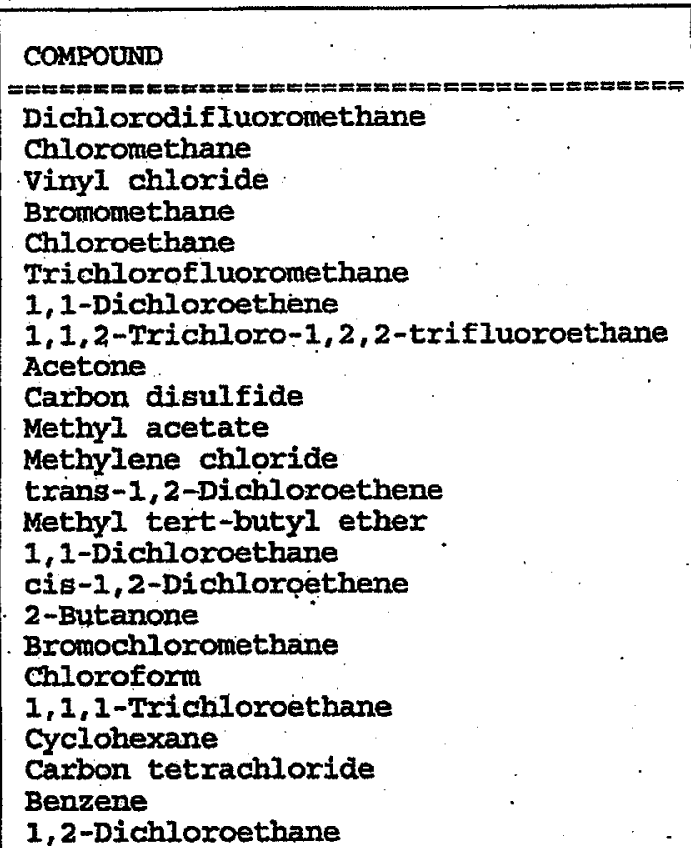 & $\begin{array}{l}\text { CONCENTRATION } \\
\mathrm{lug} / \mathrm{L} \text { or } \mathrm{ug} / \mathrm{k} \\
=========\end{array}$ & $\begin{array}{c}\text { UNITS: } \\
\mathrm{kg}) \underline{\mathrm{ug} / \mathrm{L}} \\
======= \\
0.50 \\
0.50 \\
0.50 \\
0.50 \\
0.50 \\
0.50 \\
0.50 \\
0.50 \\
1.9 \\
0.50 \\
0.50 \\
0.50 \\
0.50 \\
0.50 \\
0.50 \\
0.50 \\
5.0 \\
0.50 \\
0.50 \\
0.50 \\
0.50 \\
0.50 \\
0.50 \\
0.50\end{array}$ & 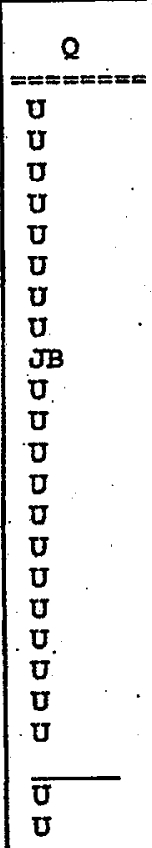 \\
\hline
\end{tabular}

Report 1,4-Dioxane for Iow-Medium VOA analysis only

SOMO1.2 
$I B$ - FORM I VOA-2

VOLATILE ORGANICS ANALYSIS DATA SHEET

EPA SAMPLE NO.

Lab Name: TESTAMERICA BURLINGTON

Lab Code: STLV Case No.: BARNES

Matrix: (SOIL/SED/WATER) Water

Sample wt/vol: 25.0

(g/mL) mL

Ievel: (TRACE/IOW/MED) TRACE

* Moisture: not dec.

GC Column: DB-624

ID: 0.53

Soil Extract Volume:

Purge volume: 25.0
Contract: $\mathbf{2 1 0 0 5}$

Mod. Ref No.:

SDG No.: 128393

Lab Sample ID: 772907

Lab File ID: 772907

Date Received: 10/24/2008

Date Analyzed: $10 / 27 / 2008$

Dilution Factor: 1.0

Soil Aliquot volume:

(ur)

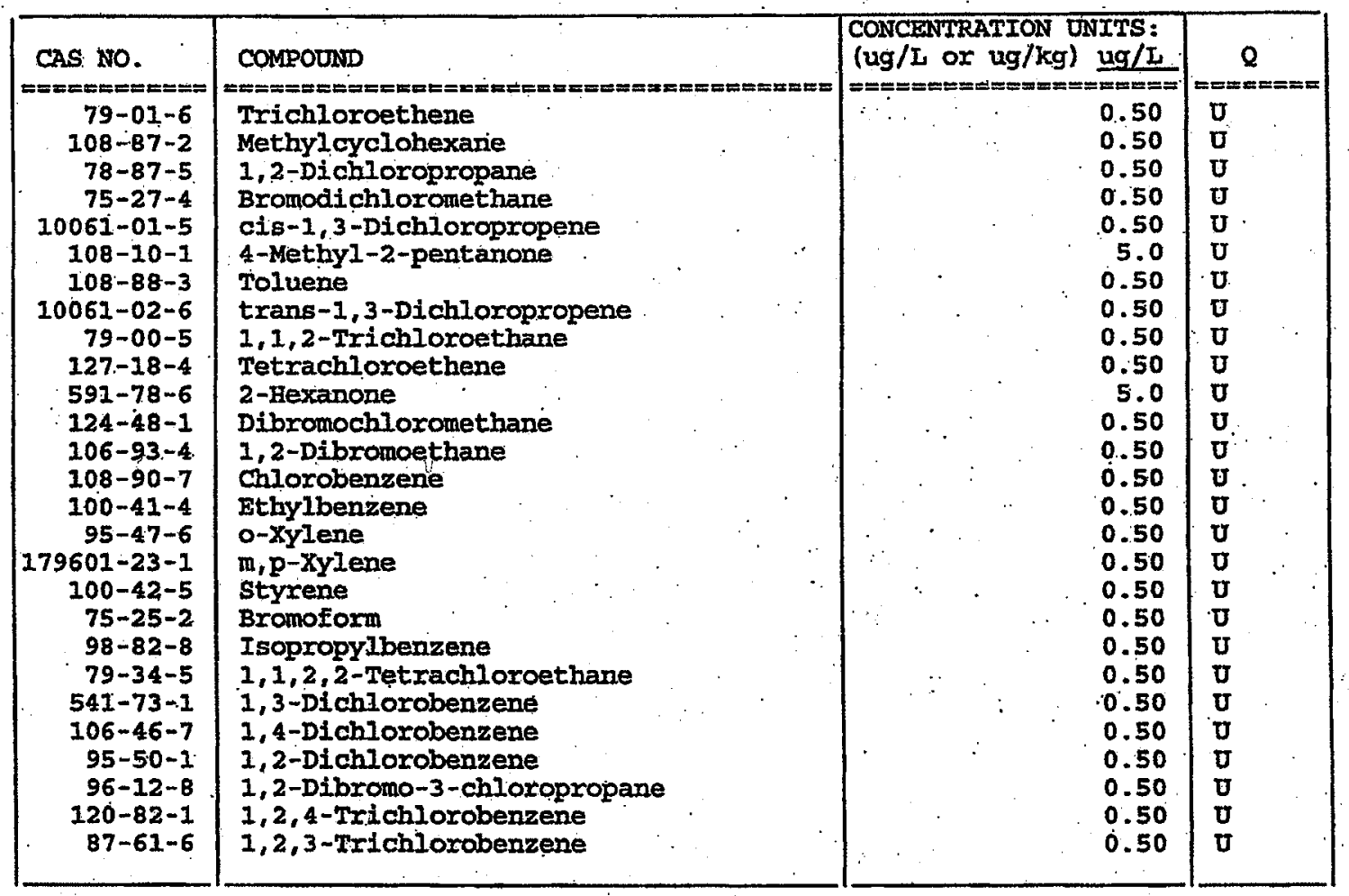

SOMO1.2 
$1 A$ - FORM I VOA-1

VOLATILE ORGANICS ANALYSIS DATA SHEET

EPA SAMPLE NO.

Lab Name: TESTAMERICA BURIINGTON

Lab Code: STLV Case NO.: BARNES

Matrix: (SOII/SED/WATER) Water

Sample wt/vol: $25.0 \quad(\mathrm{~g} / \mathrm{mL}) \mathrm{mL}$

LeVel: (TRACE/LOW/MED) TRACEE

\% Moisture: not dec.

GC Column: DB-624

ID: 0.53

Soil Extract Volume:

Purge volume: 25.0
Contract: 21005

Mod. Ref No. :

SDG NO. : 128393
Lab Sample ID: 772906

Lab. File ID: 772906

Date Received: 10/24/2008

Date Analyzed: 10/27/2008

Dilution Factor: 1.0

Soil Aliquot Volume:

(uL)

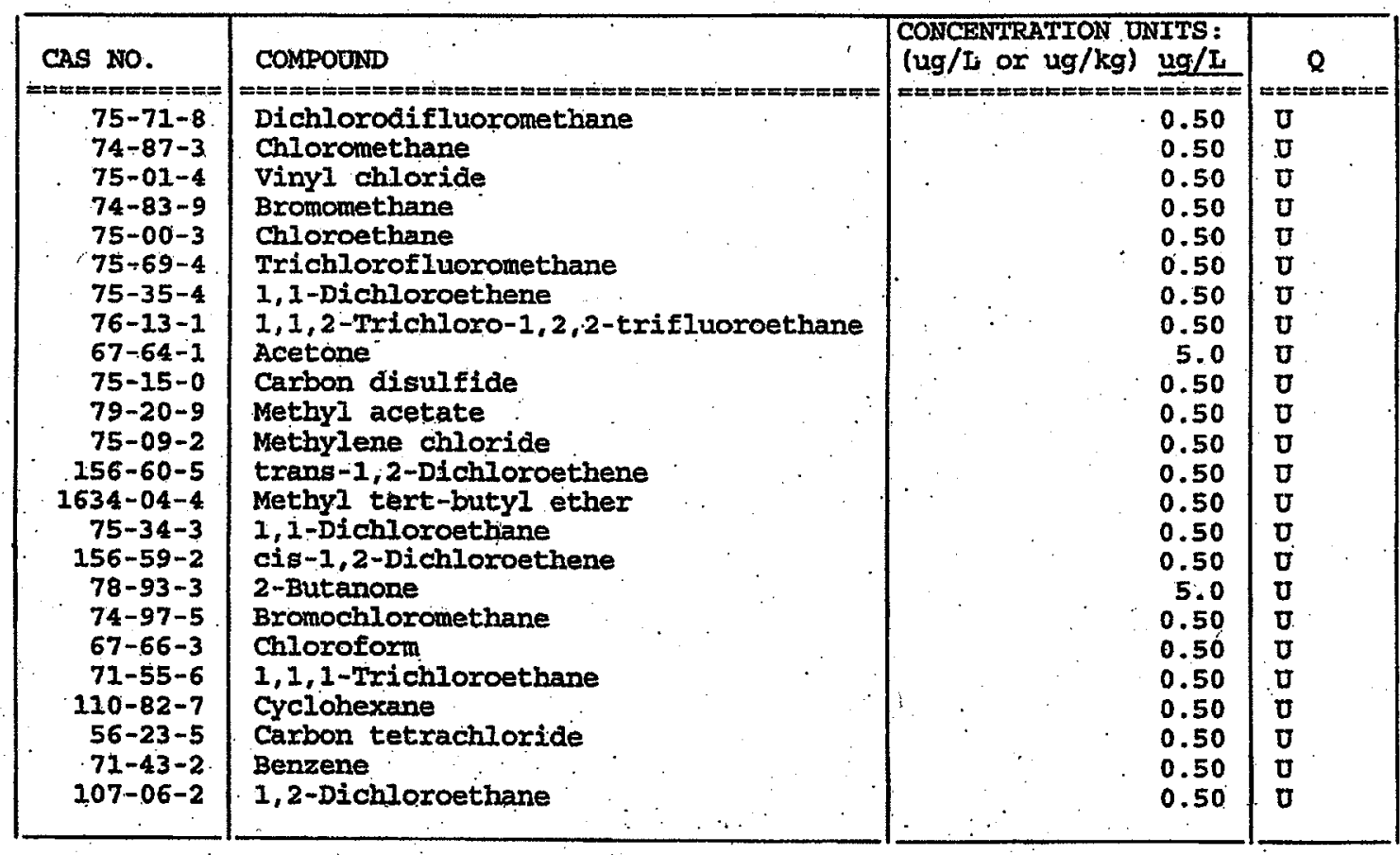

Report 1,4-Dioxane for Low-Medium VOA analysis only

SOMOI.2 
1B - EORM I VOA-2

VOIATIIE ORGANICS ANALYSIS DATA SEEET

EPA SAMPLE NO.

Lab Name: TESTAMERICA BURIINGTON

Contract: 21005

Lab code: STLV Case No.: BARNES

Mod. Ref No.:

SDG NO.: 128393

Matrix: (SOIY/SED/WATER) Water

Sample wt/vol: $25.0 \quad(\mathrm{~g} / \mathrm{mL}) \mathrm{mL}$

LEVeI: (TRACE/LOW/MRD) TRACE

\% Moisture: not dec.

GC Column: DB-624

soil Extract Volume:

ID: 0.53

(mm)

(uL)

(mL)

Punge volume: 25.0
Lab Sample ID: 772906

Lab File ID: 772906

Date Received: $10 / 24 / 2008$

Date Analyzed: 10/27/2008

Dilution Factor: 1.0

Soil Aliquot Volume:

(uL)

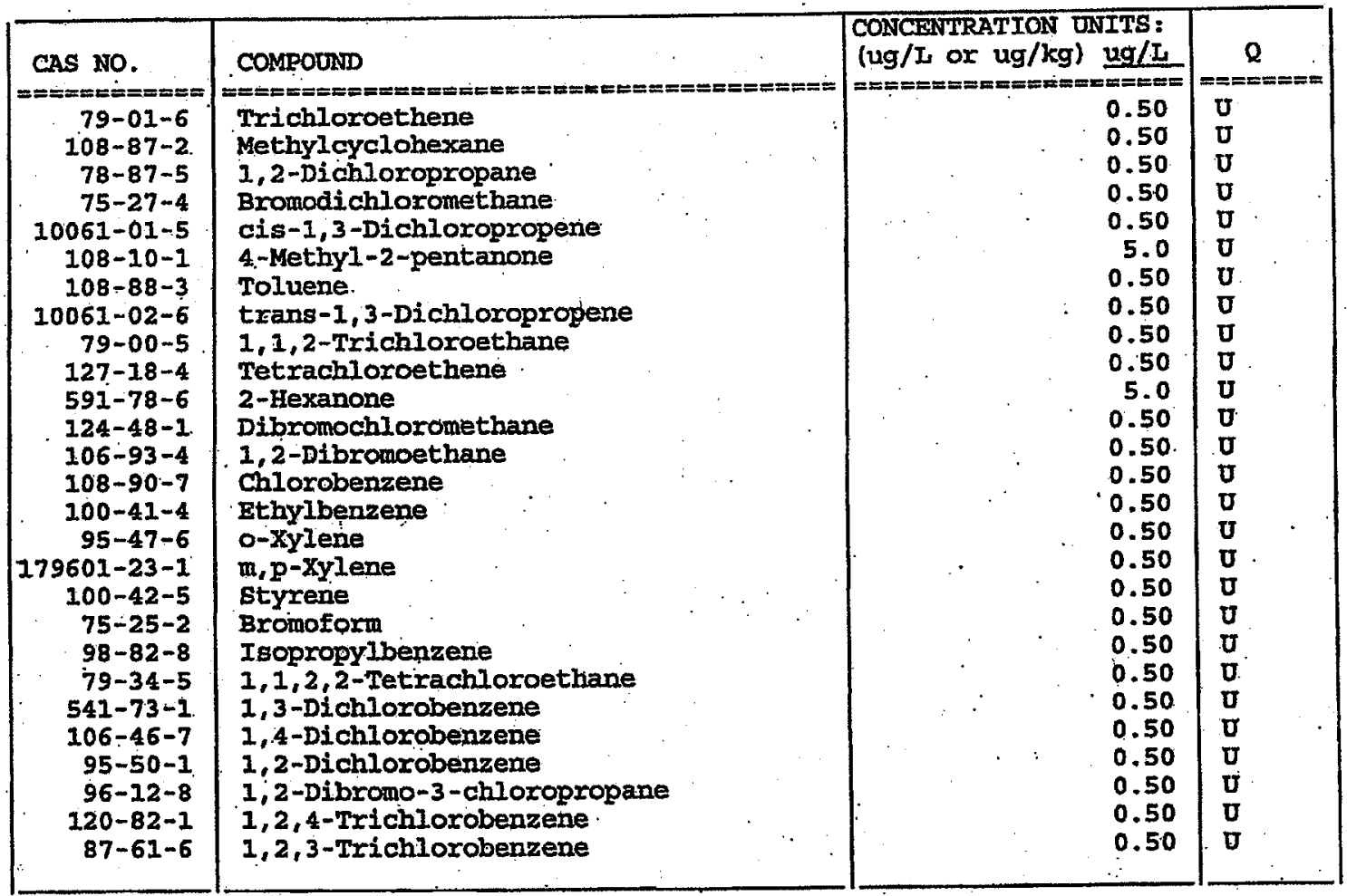

SOMOI. 2 
$1 A$ - FORM I VOA-1

VOIAATIIE ORGANICS ANALYSIS DATA SHEET

EPA SAMPLE NO.

BAMW7W27727

Lab Name: TESTAMERICA BURLINGTON

Contract: 21005

Lab Code: STLV Case No.: BARNES

Mod. ReE No.:

SDG NO.: 128393

Matrix: (SOII/SED/WATER) Water

Sample wt/vol: 25.0

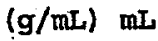

Level: (TRACE/LOW/MED) TRACE

\%oisture: not dec.

ID: 0,53

GC Column: DB-624

Soil Extract Volume:

Purge volume: 25.0
Lạb Sample ID: 77.2908

Lab File ID: 772908

Date Received: 10/24/2008

Date Analyzed: $10 / 27 / 2008$

(mm) Dilution Factor: 1.0

(iu) Soil Aliquot volume:

(UiT)

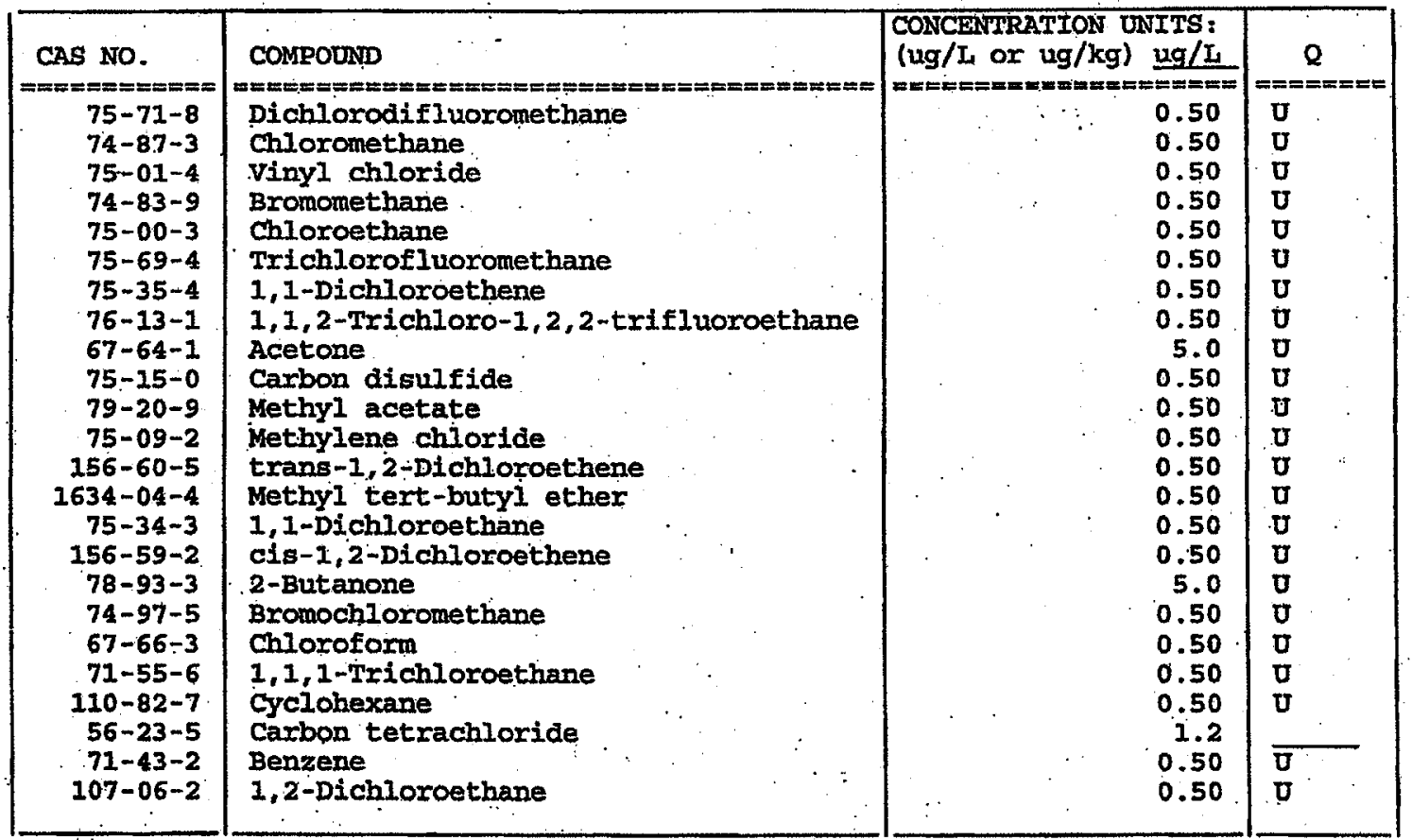

Report 1,4-Dioxane fox Low-Medium VOA analysis only 
1B - FORM I VOA-2

EPA SAMPLE NO.

VOTATILE ORGANICS ANALYSIS DATA SHEET

BAMW7W27727

Lab Name: TESTAMERICA BURLINGTON

contract: 21005

Lab code: STLV Case No.: BARNES

Mod. Ref No.:

SDG NO.: 128393

Matrix: (SOIL/SED/WATER) Water

Sample wt/vol: 25.0

$(\mathrm{g} / \mathrm{mL}) \mathrm{mL}$

Level: (TRACE/LOW/MED) TRACB

\% Moisture: not dec.

GC Column: $D B-624$

ID: 0.53

Soil Extract Volume:

Purge Volume: 25.0
Lab Sample ID: $\mathbf{7 7 2 9 0 8}$

Lab File ID: 772908

Date Received: $10 / 24 / 2008$

Date Analyzed: $10 / 27 / 2008$

(mm) Dilution Factor: 1.0

(uL)

Soil Aliquot volume:

(uT)

(mI)

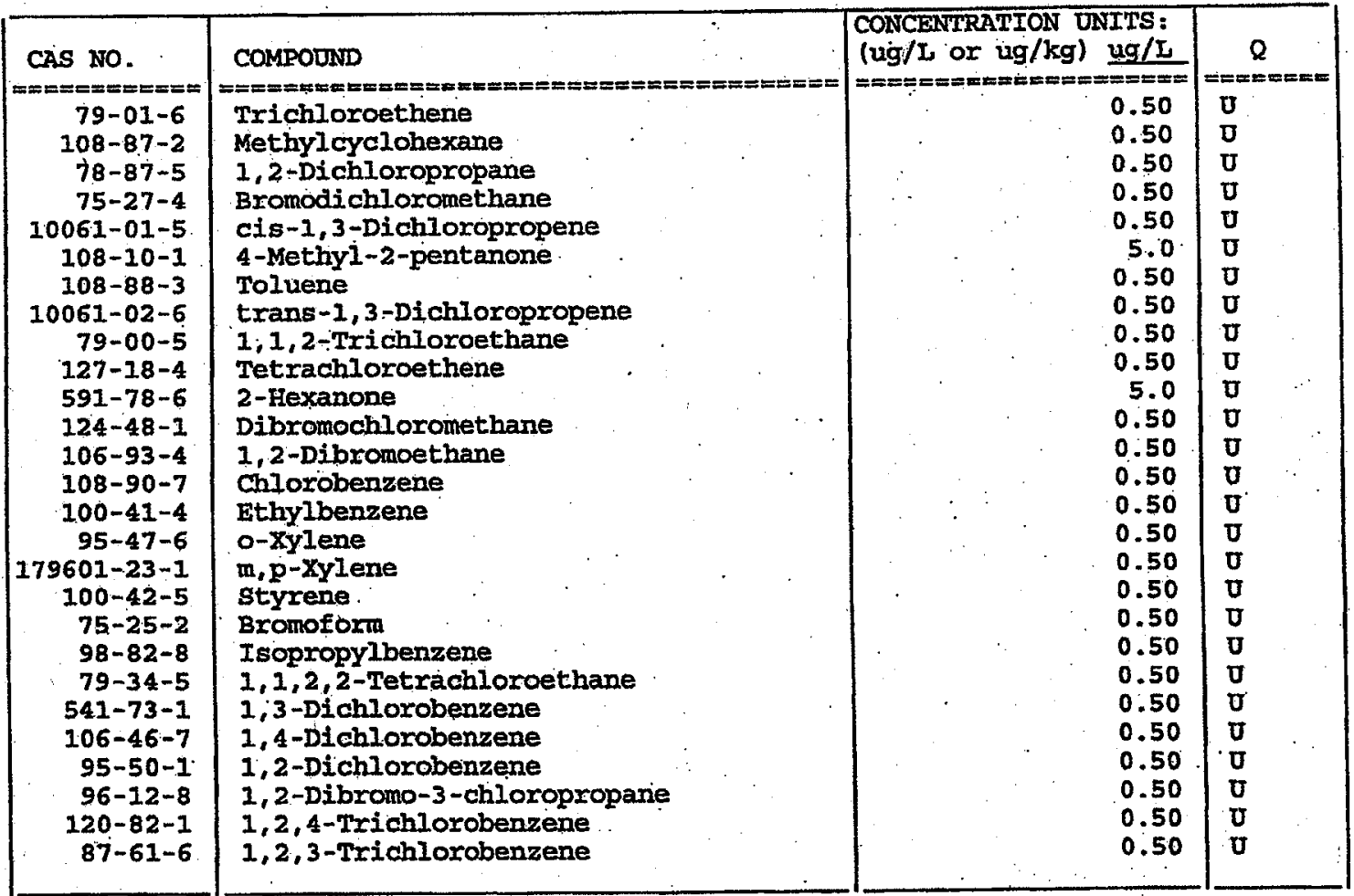

SOMO1.2 
$1 A$ - FORM I VOA-1

VOLATILE ORGANICS ANALYSIS DATA SHEET

EPA SAMPLE NO.

BAPWS2W27748

Iab Name: TESTAMERICA BURIINGTON

Contract: 21005

Lab code: STLV Case No.: BARNES

Mod. Ref No.:

SDG No.: 128393

Matrix: (SOIL/SED/WATER) Water

Sample wt/vol: $25.0 \quad(\mathrm{~g} / \mathrm{mL}) \mathrm{mL}$

Level: (TRACE/LOW/MED) TRACE

\%oisture: not dec.

GC Column: DB-624

ID: 0.53

Soil Extract Volume:

Purge volume: 25.0
Lab Sample ID: $\mathbf{7 7 2 9 1 0}$

Lab File ID: 772910

Date Received: 10/24/2008

Date Analyzed: $10 / 27 / 200 \mathrm{~B}$

Dilution Factor: 1.0

Soil Aliquot volume:

(ut)

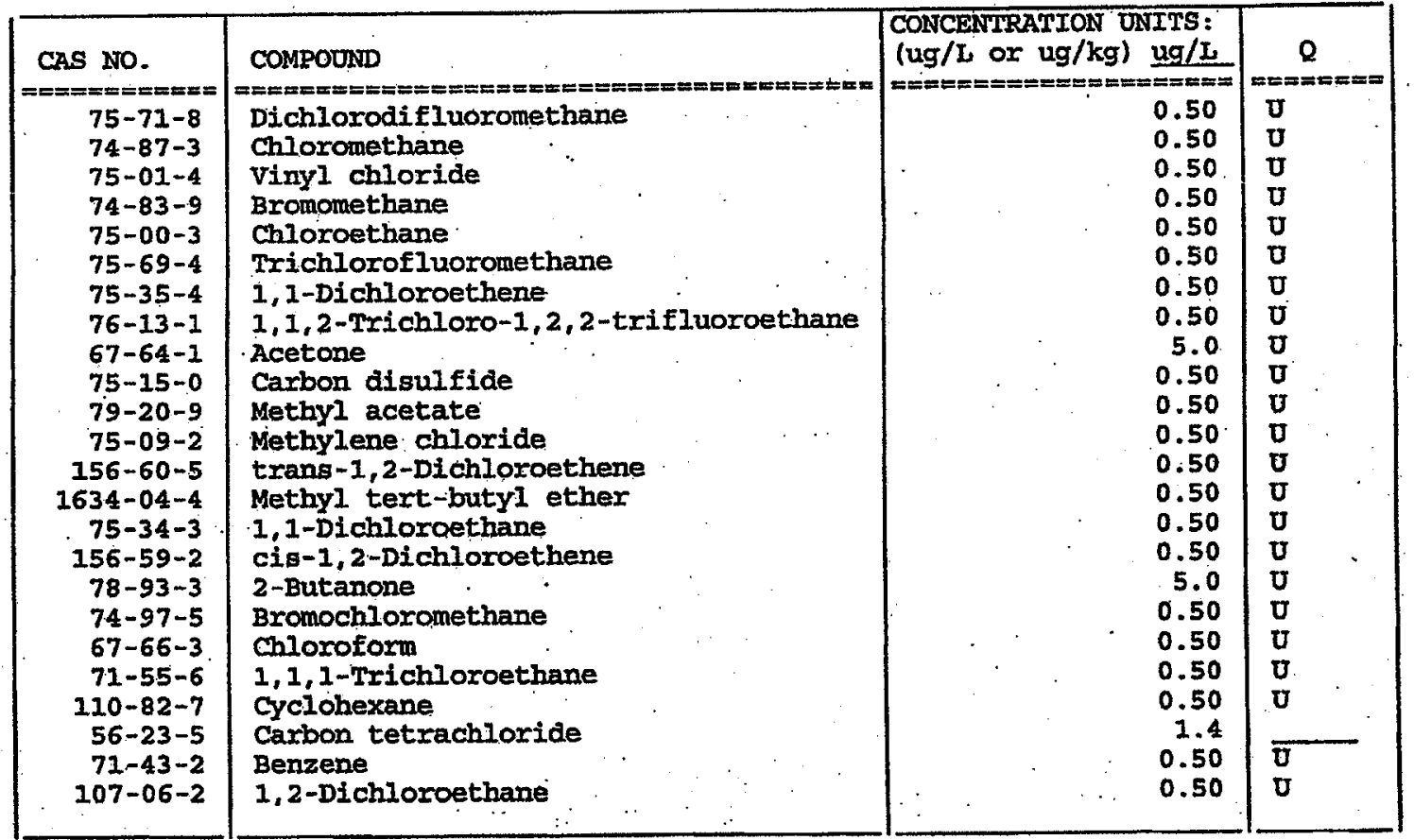

Report 1,4-Dioxane for Low-Medium VOA analysis only

SOMOI:2 
$1 B$ - FORM I VOA-2

VOLATILE ORGANICS ANAIYSIS DATA SHEET

EPA SAMPLE NO. BAPWS2W27748
Lab Name: TESTAMERICA BURLINGTON

Lab Code: STLV

Case NO.: BARNES

Matrix: (SOIL/SED/WATER) Water

Sample wt/vol: $25.0 \quad(\mathrm{~g} / \mathrm{mL}) \mathrm{mL}$

Level: (TRACE/LOW/MED) TRACE

Moisture: not dec.

GC Column: DB-624

ID: 0.53

Soil Extract Volume:

Purge Volume: 25.0
Contract: 21005

Mod. Ref No.:
SDG NO.: 128393
Lab Sample ID: 772910

Lab File ID: 772910

Date Received: 10/24/2008

Date Analyzed: 10/27/2008

Dilution Factor: 1.0

(ul)

(uf) Soil Aliquot Volume:

(mu)

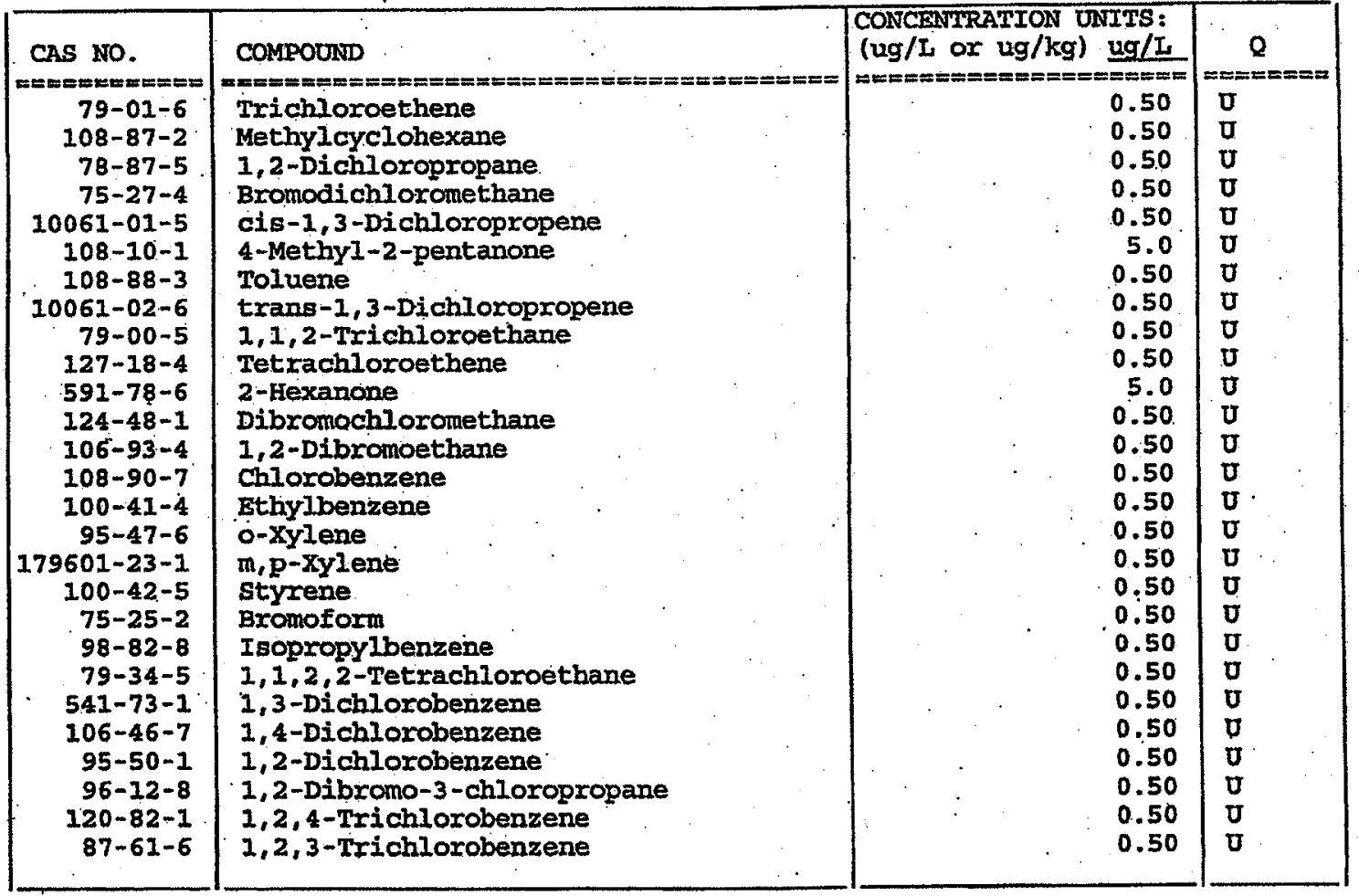

SOMO1.2 
$1 A$ - FORM I VOA-

VOLATILE ORGANICS ANALYSIS DATA SHEET

EPA SAMPLE NO.

Iab Name: TESTAMERICA BURLINGTON

Contract: 210.05

Lab Code: STLV Case No.: BARNES

Mod. Ref No.:

SDG NO.: 128393

Matrix: (SOIL/SED/WATER) Water

Sample wt/vol: $25.0 \quad(\mathrm{~g} / \mathrm{mL}) \mathrm{mL}$

Level: (TRACE/LOW/MED) TRACE

: Moisture: not dec.

GC Column: DB-624

ID: 0.53

Soil Extract Volume:

Purge volume: 25.0
Lab Sample ID: 772911

Lab File ID: 772911

Date Received: $10 / 24 / 2008$

Date Analyzed: 10/27/2008

(mm) Dilution Factor: 1.0

(uL)

Soil Aliquot Volume:

(III)

\begin{tabular}{|c|c|}
\hline $\begin{array}{r}\text { CAS NO. } \\
75-71-8 \\
74-87-3 \\
75-01-4 \\
74-83-9 \\
75-00-3 \\
75-69-4 \\
75-35-4 \\
76-13-1 \\
67-64-1 \\
75-15-0 \\
79-20-9 \\
75-09-2 \\
156-60-5 \\
1634-04-4 \\
75-34-3 \\
156-59-2 \\
78-93-3 \\
74-97-5 \\
67-66-3 \\
71-55-6 \\
210-82-7 \\
56-23-5 \\
71-43-2 \\
107-06-2\end{array}$ & 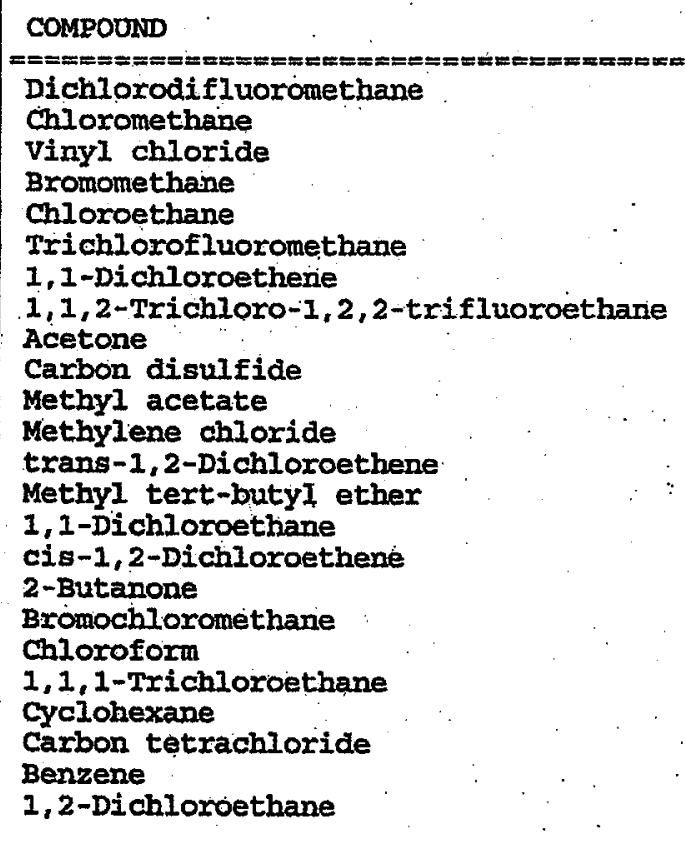 \\
\hline
\end{tabular}

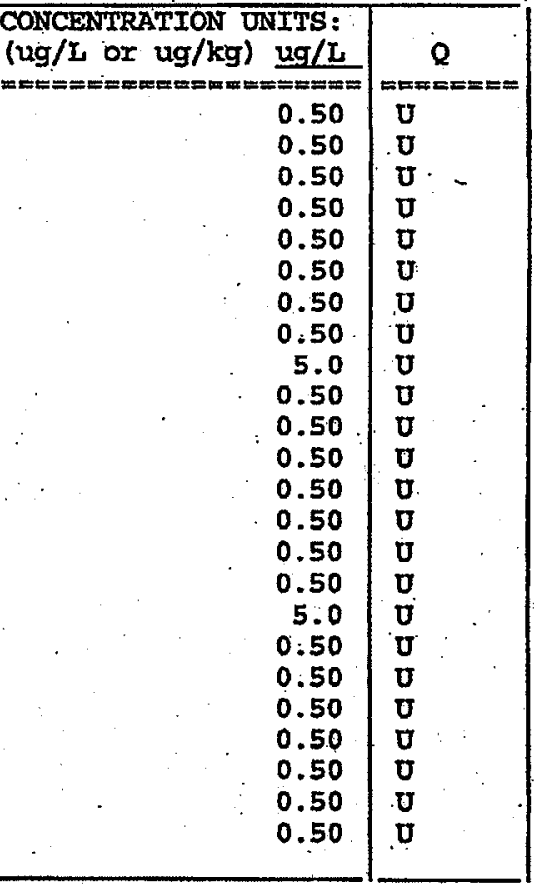

Report 1,4-Dioxane for Iow-Medium VOA analysis only

SOMO1. 2 
IB - FORM I VOA-2

VOTATILE ORGANICS ANALYSIS DATA SHEET
EPA SAMPLE NO.

BAQCTBW27755
Lab Name: TESTAMGRICA BURLINGTON

Lab Code: STLV Case No.: BARNES

Matrix: (SOIL/SED/WATER) Water

Sample wt/vol: 25.0

(g/mL) $\mathrm{mL}$

Ievel: (TRACE/IOW/MED) TRACE

\% Moisture: not dec.

GC Column: DB-624

Soil Extract Volume:

$I D: 0.53$

(min)

(uL)

Contract: 21005

Mod. Ref No.:

Lab File ID: .772911

Purge volume: 25.0

Lab. Sample ID: 772911

Date Received: $10 / 24 / 2008$

Date Analyzed: $10 / 27 / 2008$

Dilution Factor: 1.0

(mL)

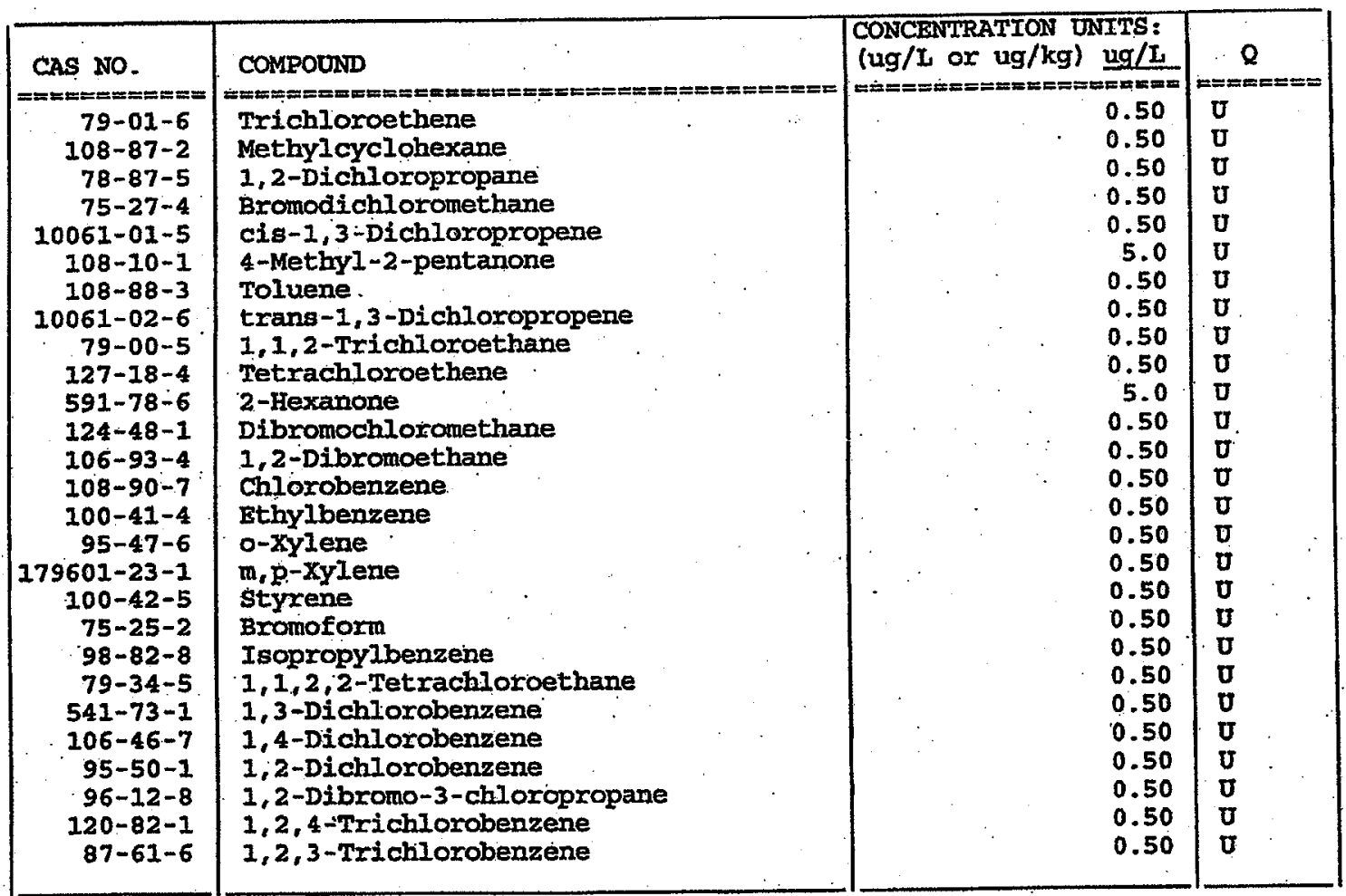

SOMO1 2 
IA - FORM I VOA-I

VOLATILE ORGANICS ANALYSIS DATA SHEET

EPA SAMPLE NO.

VABLKO1
Lab Name: TESTAMERICA BURLINGTON

Lab Code: STLV

Case No.: BARNES

Matrix: (SOIL/SED/WATER) Water

Sample wt/vol: 25.0

$(\mathrm{g} / \mathrm{mL}) \mathrm{mL}$

IEVel: (TRACE/IOW/MED) TRACE

\% Moisture: not dec.

GC Column: $\mathrm{DB}-624$

ID: 0.53
Contract: 21005

Mod. Ref No.:
SDG NO.: 128393
Soil Extract Volume:

Purge Volume: 25.0
Lab Sample ID: 772912 .

Lab File ID: 772912

Date Received:

Date Analyzed: $10 / 27 / 2008$

(mm) Dilution Factor: 1.0

(ư) Soil Aliguot volume:

(ut)

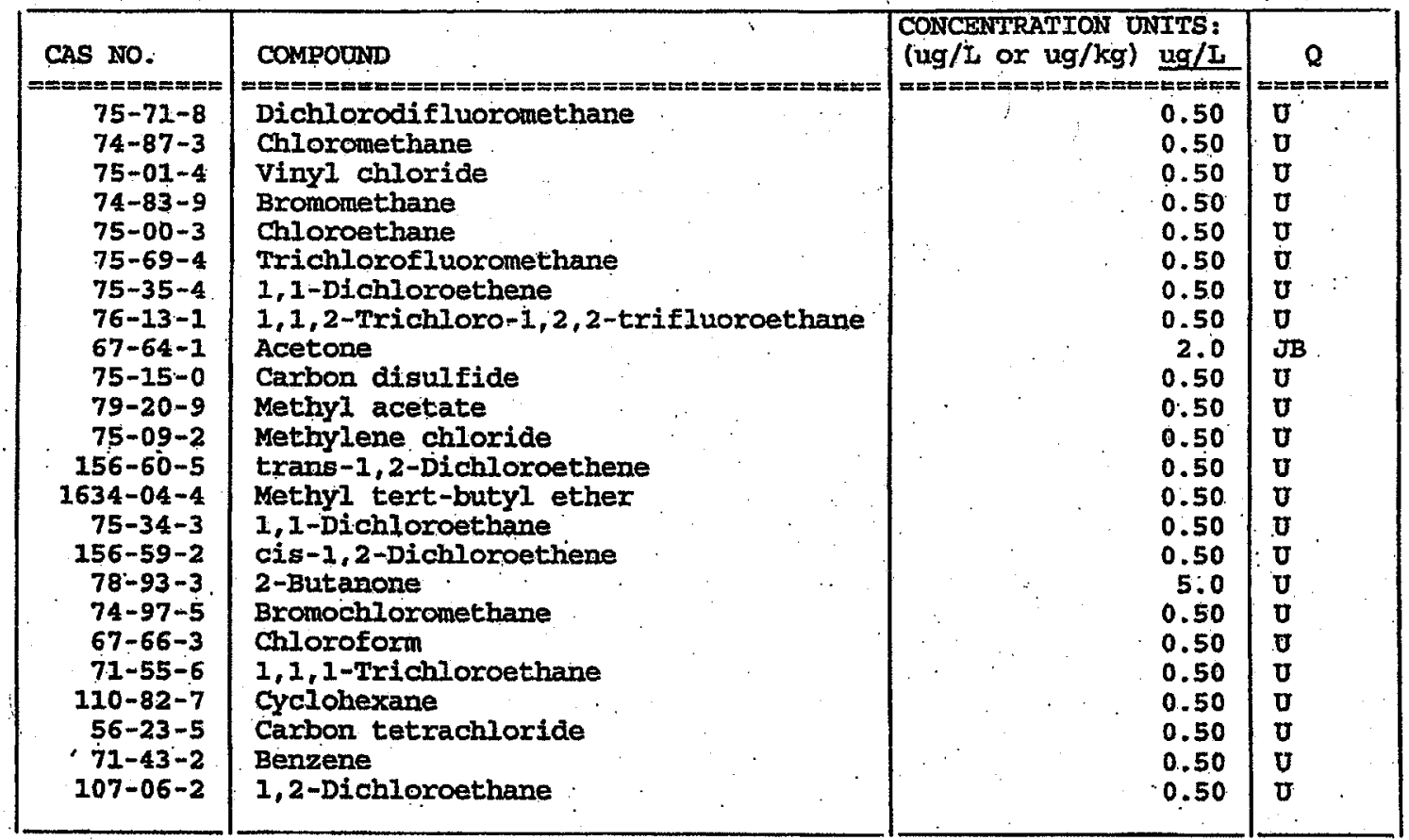

Report 1,4-Dioxane for Low-Medium VOA analysis only

SOMO1.2 
1B - FORM I VOA-2

VOLATITE ORGANICS ANALYSIS DATA SHEET

EPA SAMPLE NO.

VHBLK01

Lab Name: TESTAMERICA BURIINGTON

Contract: 21005

Lab code: STIV Case No.: BARNES

Mod. Ref No.

SDG No.: 128393

Matrix: (SOIL/SED/WATER) Water

Lab Sample ID: 772912

Sample wt/vol: 25.0

(g/mL) mr

Lab File ID: 772912

LeVel: (TRACE/LOW/MED) TRACE

Date Received:

Moisture: not dec.

Date Analyzed: $10 / 27 / 2008$

GC Column: DB-624

ID: 0.53

(mm)

Dilution Factor: 1.0

Soil Extract Volume:

(ur) Soil Aliquot volume:

(ut)

Purge Volume: 25.0

(mL)

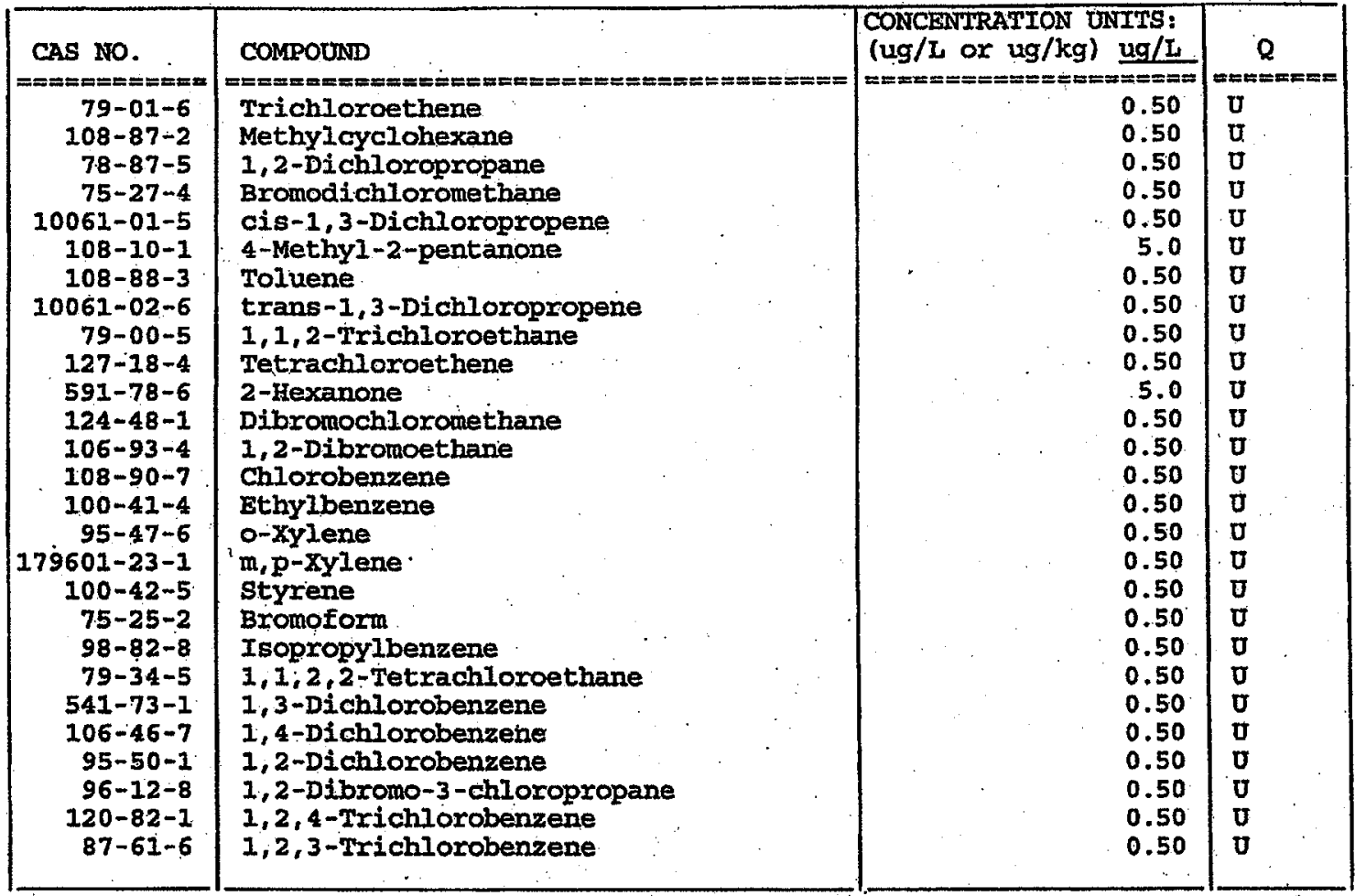

SOMO1.2 


\section{Appendix E:}

Results of City of Barnes Sampling of Public Wells in 1986-2008 


\begin{tabular}{|c|c|c|c|c|c|c|c|c|c|}
\hline FDERALID & STATEID & SYSTEM NAME & $\begin{array}{l}\text { FAQUTY } \\
\text { NAME }\end{array}$ & $\begin{array}{c}\text { CUECTION } \\
\text { DATE }\end{array}$ & $\begin{array}{l}\text { ANALYTE } \\
\text { CODE }\end{array}$ & ANALYTENAME & RESULT & \begin{tabular}{|c|} 
UNIT OF \\
MEASURE
\end{tabular} & COMMENTS \\
\hline KS2020110 & B4500 & BARNES, GTYOF & TP001 & $8 / 3 / 2008$ & 2982 & CARBON TEIRACHLORIDE & 0.89 & UG/L & WE 12 \\
\hline KS2020110 & B4500 & BARNES, GTY OF & TP002 & $7 / 14 / 2008$ & 2982 & CARBON TEIRACHLORIDE & 0 & & W日 $\perp 3$ \\
\hline KS2020110 & B4500 & BARNES, GTYOF & TP001 & $7 / 14 / 2008$ & 2982 & CARBON TEIRACHLORIDE & 1.3 & UG/L & W日 12 \\
\hline KS2020110 & B4500 & BARNES, GTY OF & TP002 & $7 / 11 / 2005$ & 2982 & CARBON TEIRACHLORIDE & 0 & & Sample taken at W日 $\perp 3$ \\
\hline KS2020110 & B4500 & BARNES, GTYOF & TP001 & $7 / 11 / 2005$ & 2982 & CARBON TEIRACHLORIDE & 0 & & Sample taken at WE $\perp 2$ \\
\hline KS2020110 & B4500 & BARNES, GTYOF & TP002 & $7 / 15 / 2002$ & 2982 & CARBON TEIRACHLORIDE & 0 & & Sample taken at W日—\#3 \\
\hline KS2020110 & B4500 & BARNES, GTYOF & TP001 & $7 / 15 / 2002$ & 2982 & CARBON TEIRACHLORIDE & 0 & & Sample taken at WE—\#2 \\
\hline KS2020110 & B4500 & BARNES, GTYOF & TP002 & $7 / 9 / 2001$ & 2982 & CARBON TEIRACHLORIDE & 0 & & Sample taken at WEL\#3 \\
\hline KS2020110 & B4500 & BARNES, GTY OF & TP001 & $7 / 9 / 2001$ & 2982 & CARBON TEIRACHLORIDE & 0 & & Sample taken at W日 $\perp \# 2$ \\
\hline KS2020110 & B4500 & BARNES, GTYOF & TP002 & $7 / 11 / 2000$ & 2982 & CARBON TEIRACHLORIDE & 0 & & Sample taken at WE丩\#3 \\
\hline KS2020110 & B4500 & BARNES, GTYOF & TP001 & $7 / 11 / 2000$ & 2982 & CARBON TEIRACHLORIDE & 0.5 & UG/L & Sample taken at WE丩\#2 \\
\hline KS2020110 & B4500 & BARNES, GTY OF & TP002 & $7 / 12 / 1999$ & 2982 & CARBON TEIRACHLORIDE & 1.7 & UG/L & Sample taken at WE丩\#3 \\
\hline KS2020110 & B4500 & BARNES, GTYOF & TP001 & $7 / 12 / 1999$ & 2982 & CARBON TEIRACHLORIDE & 1.2 & UG/L & Sample taken at WE $\perp \# 2$ \\
\hline KS2020110 & B4500 & BARNES, ATY OF & TP001 & $8 / 4 / 1998$ & 2982 & CARBON TEIRACHLORIDE & 1.5 & UG/L & Sample taken at GTY OFBARNESSAMPL \\
\hline KS2020110 & B4500 & BARNES, ATYOF & TP002 & $8 / 27 / 1997$ & 2982 & CARBON TEIRACHLORIDE & 0 & & Sample taken at GTY OFBARNES- W日 \\
\hline KS2020110 & B4500 & BARNES, GTYOF & TP001 & $8 / 27 / 1997$ & 2982 & CARBON TEIRACHLORIDE & 0.9 & UG/L & Sample taken at GTY OFBARNES- W日 \\
\hline KS2020110 & B4500 & BARNES, GTYOF & TP002 & $8 / 13 / 1996$ & 2982 & CARBON TEIRACHLORIDE & 0.5 & UG/L & Sample taken at SAMPLNG TAPATWE \\
\hline KS2020110 & B4500 & BARNES, GTYOF & TP001 & $8 / 13 / 1996$ & 2982 & CARBON TEIRACHLORIDE & 1.3 & UG/L & Sample taken at SAMPLNG TAPATWEI \\
\hline KS2020110 & B4500 & BARNES, GTY OF & TP001 & $7 / 25 / 1995$ & 2982 & CARBON TEIRACHLORIDE & 1.1 & UG/L & Sample taken at BARNESWE丩\#2 \\
\hline KS2020110 & B4500 & BARNES, GTYOF & TP002 & $5 / 3 / 1995$ & 2982 & CARBON TEIRACHLORIDE & 0 & & Sample taken at W日レ\#3 00005272 \\
\hline KS2020110 & B4500 & BARNES, GTYOF & TP001 & $5 / 3 / 1995$ & 2982 & CARBON TEIRACHLORIDE & 0.5 & UG/L & Sample taken at W日上\#2 00040848 \\
\hline KS2020110 & B4500 & BARNES, GTYOF & 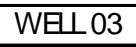 & $8 / 12 / 1991$ & 2982 & CARBON TEIRACHLORIDE & 0 & & Sample taken at 480 FT W OFMAIN ST \\
\hline KS2020110 & B4500 & BARNES, GTYOF & W日 $\perp 02$ & $8 / 12 / 1991$ & 2982 & CARBON TEIRACHLORIDE & 0 & & Sample taken at WE $\perp$ LOCATED IN THE \\
\hline KS2020110 & B4500 & BARNES, GTYOF & W日 $\perp 03$ & $1 / 30 / 1989$ & 2982 & CARBON TEIRACHLORIDE & 0 & & Sample taken at 480 FT W OFMAIN ST \\
\hline KS2020110 & B4500 & BARNES, GTYOF & 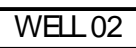 & $1 / 30 / 1989$ & 2982 & CARBON TEIRACHLORIDE & 0 & & Sample taken at WE $\perp$ LOCATED IN THE \\
\hline KS2020110 & B4500 & BARNES, GTYOF & $\mathrm{WE} \perp 03$ & 9/2/1988 & 2982 & CARBON TEIRACHLORIDE & 0 & & Sample taken at 480 FT W OFMAIN ST \\
\hline KS2020110 & B4500 & BARNES, GTYOF & 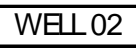 & 9/2/1988 & 2982 & CARBON TEIRACHLORIDE & 0 & & 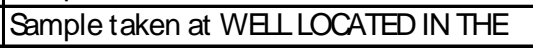 \\
\hline KS2020110 & B4500 & BARNES, GTYOF & WE丩03 & $7 / 7 / 1987$ & 2982 & CARBON TEIRACHLORIDE & 2.1 & UG/L & Sample taken at 480 FTW OFMAIN ST \\
\hline KS2020110 & B4500 & BARNES, ATYOF & WE $\perp 02$ & $7 / 7 / 1987$ & 2982 & CARBON TEIRACHLORIDE & 2.5 & UG/L & Sample taken at WE $\perp$ LOCATED IN THE \\
\hline KS2020110 & B4500 & BARNES, GTYOF & $\mathrm{WE} \perp 03$ & $4 / 8 / 1986$ & 2982 & CARBON TEIRACHLORIDE & 0.5 & UG/L & Sample taken at 480 FT W OFMAIN ST \\
\hline KS2020110 & B4500 & BARNES, GTYOF & W日ل 02 & $4 / 8 / 1986$ & 2982 & CARBON TEIRACHLORIDE & 2.1 & UG/L & Sample taken at WE— LOCATED IN THE \\
\hline
\end{tabular}


Argonne

Environmental Science Division

Argonne National Laboratory

9700 South Cass Avenue, Bldg. 203

Argonne, IL 60439-4843

www.anl.gov 